\title{
Systems of classical particles in the grand canonical ensemble, scaling limits and quantum field theory
}

\author{
Sergio Albeverio and Hanno Gottschalk \\ Institut für angewandte Mathematik, \\ Rheinische Friedrich-Wilhelms-Universität Bonn, \\ Wegelerstr. 6, D-53115 Bonn, Germany \\ e-mail: albeverio@uni-bonn.de / gottscha@wiener.iam.uni-bonn.de \\ Minoru W. Yoshida \\ Department of Mathematics and Systems Engeneering \\ The University of Electrocommunications 1-5-1 \\ Chofugaku, Tokyo 182-8585, Japan \\ e-mail: yoshida@se.uec.ac.jp
}

\begin{abstract}
Euclidean quantum fields obtained as solutions of stochastic partial pseudo differential equations driven by a Poisson white noise have paths given by locally integrable functions. This makes it possible to define a class of ultra-violet finite local interactions for these models (in any space-time dimension). The corresponding interacting Euclidean quantum fields can be identified with systems of classical "charged" particles in the grand canonical ensemble with an interaction given by a nonlinear energy density of the "static field" generated by the particles' charges via a "generalized Poisson equation". A new definition of some well-known systems of statistical mechanics is given by formulating the related field theoretic local interactions. The infinite volume limit of such systems is discussed for models with trigonometric interactions using a representation of such models as Widom-Rowlinson models associated with a (formal) Potts models at imaginary temperature. The infinite volume correlation functional of such Potts models can be constructed by a cluster expansion. This leads to the construction of extremal Gibbs measures with trigonometric interactions in the low-density high temperature (LD-HT) regime. For Poissonian models with certain trigonometric interactions an extension of the well-known relation between the (massive) sine-Gordon model and the Yukawa particle gas connecting characteristic- and correlation functionals is given and used to derive infinite volume measures for interacting Poisson quantum field models through an alternative route. The continuum limit of the particle systems under consideration is also investigated and the formal analogy with the scaling limit of renormalization group theory is pointed out. In some simple cases the question of (non-) triviality of the continuum limits is clarified.
\end{abstract}

Keywords: Euclidean quantum field theory, Poisson random fields, local interactions, particle systems in the grand canonical ensemble, correlation functionals, Potts- and 
Widom-Rowlinson models, cluster expansion, extremal Gibbs measures, continuum limit of particle systems, sine-Gordon model.

MSC (2000): $\underline{81 \mathrm{~T} 08}, 60 \mathrm{G} 55,60 \mathrm{G} 60,81 \mathrm{~T} 10,82 \mathrm{~B} 21,82 \mathrm{~B} 28$

\section{Introduction}

Strong connections between classical statistical mechanics and quantum field theory have been established in the framework of Euclidean quantum field theory (EQFT), see e.g. [1, 34, 55]. In particular this applies to the approximation of Euclidean quantum fields by lattice spin systems [34, 55], the representation as a gas of interacting random walks [1, 26, 59], or the connection of quantum field models with trigonometric interaction (e.g. the sine-Gordon model) with the gas of particles interacting through Yukawa- or Coulomb forces 9, 10, 24, 27, 28, 29. In this way, cluster expansions or correlation inequalities from classical particleor ferromagnetic spin systems have been applied to the solution of the infra-red problem in Euclidean quantum field theory.

Basically, all these constructions concern models of quantum fields given by a classical Euclidean action functional $S(X)=S^{0}(X)+\beta V_{\Lambda}(X)$ with the free term $S^{0}(X)=\frac{1}{2} \int_{\mathbb{R}^{d}}\left[|\nabla X|^{2}+m^{2} X^{2}\right] d x$ and the interaction term $V_{\Lambda}(X)$ being an additive functional in the infra-red regularizer $\Lambda \subseteq \mathbb{R}^{d}$ of local type, i.e. $V_{\Lambda}(X)=\int_{\Lambda} v(X) d x$ for some function $v: \mathbb{R} \rightarrow \mathbb{R}$. Inserting this into a heuristic path integral of Feynman type, one gets the well-known heuristic formula for the vacuum expectation values of the relativistic quantized field continued to imaginary times (Schwinger functions) as

$$
S_{\Lambda, n}\left(y_{1}, \ldots, y_{n}\right)=\frac{1}{Z_{\Lambda}} \int X\left(y_{1}\right) \cdots X\left(y_{n}\right) e^{-S^{0}(X)-\beta V_{\Lambda}(X)} \mathcal{D} X
$$

where $y_{1}, \ldots, y_{n} \in \mathbb{R}^{d}, \beta \in \mathbb{R}, m_{(-)}^{2}>$. While the path integral itself makes sense $-e^{-S^{0}(X)} \mathcal{D} X / Z_{\emptyset}$ can be identified with the Gaussian measure with covariance operator $\left(-\Delta+m^{2}\right)^{-1}$, i.e. the Nelson free field measure (for $m^{2}=0, d=1$ the Wiener measure) - it is difficult to define the interaction term $V_{\Lambda}(X)$, since the field configurations $X$ in the support of the Gaussian measure are functions only if $d=1$ : If $d \geq 2$ the field configurations in the integral (11) generically are distributions and expressions as $v(X)$ are ill-defined. This also limits the EQFT approach essentially to space-time dimension $d=1$ or $d=2$, where $v(X)$ for polynomial, trigonometric or exponential $v$ can be regularized by Wick-ordering (for the construction of the $\phi^{4}$-model in $d=3$ dimensions see [35]).

In the present paper we suggest to replace Nelson's measure $e^{-S_{0}(X)} \mathcal{D} X / Z_{\emptyset}$ in (11) by a convoluted Poisson noise measure [3]. Since Nelson's measure can be seen as a convoluted Gaussian white noise measure, from a mathematical point 
of view it is natural to generalize Equation (17) to Poisson path space measures. Furthermore, given the fact that convoluted Poisson white noise measures have support on locally integrable field configurations $X$, for a certain class of functions $v$ we can define potentials $V_{\Lambda}(X)$ without any ultra-violet renormalization (not even Wick-ordering) independently of the dimension $d \geq 2$. As we will show in Section 4, the Euclidean quantum field models obtained in this way can be identified with systems of classical continuous and interacting particles in the grand canonical ensemble. Since at least in principle the perturbed Gaussian free field models can be recovered from the related interacting "Poissonian" quantum field models by a scaling limit of the associated particle system, we can consider the above replacement as a new approximation of EQFTs by systems of statistical mechanics. Also, properties of EQFT, as e.g. Euclidean invariance, are preserved in the infinite volume limit $\Lambda \uparrow \mathbb{R}^{d}$. In this sense this new approximation takes care of important structural aspects of EQFT (which are violated e.g. by the lattice approximation, discussed e.g. in [1, 34, 55]).

Another motivation for our suggestion is the constructive approach to quantized gauge type fields developed in [3-6,10-12]: The basic framework in these references is the one of covariant stochastic partial (pseudo) differential equations driven by noise not necessarily of the Gaussian type, in contrast to Nelson's Euclidean approach [47, 48, which can be considered in the framework of stochastic partial (pseudo) differential equations of the Gaussian type. This approach started in the study of quaternionian vector [11, 12, 13, 15] and scalar models [3, 4, 16, it has then extended to much more general fields, see [6, 19, 32, 33, 39, 41]. In these cases the axiomatic framework for the relativistic fields to be accommodated, when possibly constructed, is the concept of quantum fields with indefinite metric [46, 57]. In fact, analytic continuation for these models from Euclidean imaginary time to relativistic real time is possible and the modified Wightman axioms [46] for quantum fields with indefinite metric can be verified explicitly [3, 6, 15, 19]. In particular, fields with interesting scattering behavior have been found in this class of models, also in the physical space-time dimension 4, cf. [2, 5, 6, 41]. Therefore the connection with relativistic quantum field theory does not get lost, if we replace the Nelson's measure by a convoluted Poisson noise measure.

An alternative way to describe the main attitude of this paper is to say that a systematic discussion is given how to introduce perturbations of the basic (indefinite metric) Euclidean quantum fields to construct other such fields. In analogy with the standard constructive approach, this is achieved by constructing Gibbs type measures for a bounded region of space-time (finite volume) and then removing this restriction in the sense of a thermodynamic limit. The main result of this paper consists in showing that such an approach indeed can be developed and yields at the same time interesting new relations with models of classical 
statistical mechanics. Some results of this work have been announced in [7].

Let us finally describe the content of the single sections of this paper: In Section 2 the basic notions of generalized white noise convoluted generalized white noise are recalled. It is also described, how the corresponding random fields lead, by analytic continuation of their moment functions (Schwinger functions), to relativistic Wightman functions satisfying all axioms of an indefinite metric quantum field theory. Some special Green's functions used to perform the convolution are discussed and the scattering behavior of the associated quantum field models is recalled. Finally, we show that the lattice approximation of the Euclidean noise fields canonically leads to the notion of a generalized white noise.

In Section 3, path properties of convoluted Poisson noise (CPN) are discussed and exploited to construct ultra-violet finite, local interactions: In Section 3.2 we recall that pure Poisson noise has paths in the space of locally finite "marked" configurations and hence convolution with an integrable kernel leads to fields with paths which are locally integrable (independently of the dimension $d \geq 2$ ), cf. Section 3.3. In Section 3.4 we then define the interaction term $V_{\Lambda}$ for any $v$ measurable s.t. $|v(t)| \leq a+b|t|$ for some $a, b>0$.

Section 4 is devoted to the connection between the particle systems in a grand canonical ensemble (GCE) and quantum fields defined by convoluted Poisson white noise with interaction. Theorem 4.1 shows the stability (in the statistical mechanics sense) of the field theoretic interaction potential for the associated system of classical, continuous particles.

In Section 5 several models of statistical mechanics are looked upon as systems of classical particles associated (in the sense of Section 4) with convoluted, interacting Poisson white noise. In particular the cases of a gas of hard spheres, particle systems with potentials of stochastic geometry or pair potentials which are positive definite fit into this framework.

Section 6 is the technical core of this work. We give a complete solution of the problem of taking the infinite volume limit of the models of quantum fields resp. statistical mechanics in the low-density high-temperature regime (LD-HT) and trigonometric interactions (cf. Section 6.1 for the definition of the interaction). This is presumably one of the first cluster expansion for a continuous particle system for an interaction that is not a pair-interaction ${ }^{1}$. The strategy is to represent such a model as the projection of a (formal) Potts model at imaginary temperature to one of its components (Widom-Rowlinson model), cf. Section 6.2. Even though such formal Potts models are only represented as complex valued mea-

\footnotetext{
${ }^{1}$ It is probably known to some experts that the cluster expansion for the standard Potts model at positive temperature leads to a construction of the ordinary Widom-Rowlinson model in the LD-HT regime (corresponding to "exponential" interactions for systems of particles with only positive charges [38]). But neither have the details been worked out, nor has the flexibility of this method in connection with "charged" or "marked" particles been realized.
} 
sures on the space of locally finite configurations with a extra mark indicating the "component" and cannot be interpreted in terms of statistical mechanics, the standard cluster expansion [52 for their correlation functionals goes through (Section 6.3). The projection to the first component then defines the correlation functional of the system with trigonometric interaction. Using standard arguments [42, 43] one can then reconstruct the associated infinite volume measure. Verification of Ruelle equations in Section 6.4 then implies that such measures are Gibbs. Cluster properties of correlation functionals in the infinite volume follow from the cluster expansion and imply ergodicity of the translation group and hence extremality of the Gibbs state (Section 6.5). The case of trigonometric interactions is analyzed in Section 6.6: We extend the previously known connection for the massive resp. massless sine-Gordon model and Yukawa resp. Coulomb gas models ("duality transformation").

The continuum (scaling) limit of interacting convoluted Poisson noise (with infra-red cut-off) is discussed in Section 7. We start with a rather general discussion of scaling limits for Poisson models and the relation with "renormalization group methods". The case of trigonometric interactions with ultra-violet cut-off is then analyzed with the related, ultra-violet regularized, perturbed free field. Triviality without an ultra-violet cut-off and without renormalization is shown in Section 7.3. In Section 7.4 the scaling limit for the $d=2$-dimensional sine-Gordon model without ultra-violet cut-off and with a coupling constant renormalization is established in the sense of formal power series.

\section{Generalized white noise and convoluted gen- eralized white noise}

In this section we introduce our notation and recall some results of [3]

\subsection{Generalized white noise}

For $d \geq 1$ we identify the $d$-dimensional Euclidean space-time with $\mathbb{R}^{d}$, by $\cdot / \mid$.| we denote the Euclidean scalar product / norm and $E(d)$ stands for the group of Euclidean transformations on $\mathbb{R}^{d}$. The space $\mathcal{S}$ is the space of real valued fast falling test functions on $\mathbb{R}^{d}$ endowed with the Schwartz topology. By $\mathcal{S}^{\prime}$ we denote it's topological dual space (space of tempered distributions). Let $\mathcal{B}\left(\mathcal{S}^{\prime}\right)$ be the Borel $\sigma$-algebra on $\mathcal{S}^{\prime}$, i.e. the $\sigma$-algebra generated by the open (in the weak topology) subsets of $\mathcal{S}^{\prime}$. Then, $\left(\mathcal{S}^{\prime}, B\left(\mathcal{S}^{\prime}\right)\right)$ is a measurable space.

A (tempered) random field over $\mathbb{R}^{d}$ by definition is a mapping from $\mathcal{S}$ into the space of real valued random variables on some probability space $X: \mathcal{S} \rightarrow$ $\operatorname{RV}(\Omega, \mathcal{B}, P)$ such that (i) $X$ is linear $P$-a.s. and (ii) $f_{n} \rightarrow f$ in $\mathcal{S} \Rightarrow X\left(f_{n}\right) \stackrel{\mathcal{L}}{\rightarrow}$ 
$X(f)$ where $\stackrel{\mathcal{L}}{\rightarrow}$ means convergence in probability law. Two processes $X_{j}, j=$ 1,2 , on probability spaces $\left(\Omega_{j}, \mathcal{B}_{j}, P_{j}\right), j=1,2$, are called equivalent in law if $P_{1}\left\{X_{1}\left(f_{1}\right) \in B_{1}, \ldots, X_{1}\left(f_{n}\right) \in B_{n}\right\}=P_{2}\left\{X_{2}\left(f_{1}\right) \in B_{1}, \ldots, \overline{\left.X_{2}\left(f_{n}\right) \in B_{n}\right\} \forall n} \in\right.$ $\mathbb{N}, f_{1}, \ldots, f_{n} \in \mathcal{S}$ and $B_{1}, \ldots, B_{n} \in \mathcal{B}(\mathbb{R})$, where $\mathcal{B}(\mathbb{R})$ stands for the Borel sigmaalgebra $^{2}$ on $\mathbb{R}$.

By Minlos' theorem [45] there is a one-to-one correspondence (up to equivalence in law) between tempered random fields and the characteristic functionals (i.e. continuous, normalized and positive definite functionals) $\mathcal{C}: \mathcal{S} \rightarrow \mathbb{C}$ given by $\mathcal{C}(f)=\mathbb{E}_{P}\left[e^{i X(f)}\right]$. Furthermore, $X$ can be realized as a coordinate process, i.e. there exists an unique probability measure $P^{X}$ on $\left(\overline{\left.\mathcal{S}^{\prime}, \mathcal{B}\left(\mathcal{S}^{\prime}\right)\right) \text { such that }}\right.$ for the random field $X_{c}(f)(\omega)=\langle\omega, f\rangle=\omega(f) \forall \omega \in \mathcal{S}^{\prime}$ and $f \in \mathcal{S}$ and $\mathbb{E}_{P}\left[e^{i X(f)}\right]=\mathbb{E}_{P^{X}}\left[e^{i X_{c}(f)}\right] \forall f \in \mathcal{S}$. In the following we drop the subscript $c$ and we adopt the general rule that a random field $X$ on the probability space $\left(\mathcal{S}^{\prime}, \mathcal{B}\left(\mathcal{S}^{\prime}\right), P^{X}\right)$ always is the coordinate process.

Let $\psi: \mathbb{R} \rightarrow \mathbb{C}$ be a Lévy-characteristic, i.e. a continuous, conditionally positive definite function $\left(\overline{\text { for } t_{j} \in \mathbb{R}, z_{j} \in \mathbb{C}}, j=1, \ldots, n\right.$ s.t. $\sum_{j=1}^{n} z_{j}=0$ we have $\left.\sum_{l, j=1}^{n} \psi\left(t_{l}-t_{j}\right) \bar{z}_{l} z_{j} \geq 0\right)$ such that $\psi(0)=0$. We set

$$
\mathcal{C}_{F}(f)=e^{\int_{\mathbb{R}^{d}} \psi(f) d x} \quad \forall f \in \mathcal{S}
$$

and we get from Theorem 6 p. 283 of [30] that $\mathcal{C}_{F}$ is a characteristic functional. The associated random field $F$ is called a generalized white noise. $F$ has infinitely divisible probability law, is invariant in law under Euclidean transformations and for $f, h \in \mathcal{S}$ such that supp $f \cap \operatorname{supp} h=\emptyset F(f)$ and $F(h)$ are independent random variables.

Provided $\psi$ is $C^{1}$-differentiable at 0 , one can derive the following representation for $\psi$ (cf. 20])

$$
\psi(t)=i a t-\frac{\sigma^{2}}{2} t^{2}+z \int_{\mathbb{R}}\left(e^{i s t}-1\right) d r(s) .
$$

Here $a \in \mathbb{R}, z, \sigma^{2} \in[0, \infty)$ and $r$ is a probability measure on $\mathbb{R}$ such that $r\{0\}=0$. The representation (3) is unique (for $z>0$ ). Using notions which are slightly different from the standard definitions, we call $r$ the Lévy measure of $\psi$ and $z$ is called the activity. The first term in (3) is called deterministic part, the second one the Gaussian part and the third one the Poisson part.

Inserting (3) into (2) we see that $F$ can be written as the sum of independent deterministic (i.e. constant), Gaussian and Poisson parts which are uniquely determined by $\psi$.

\footnotetext{
${ }^{2}$ The sigma-algebra generated by the open subsets.
} 


\subsection{Convoluted generalized white noise}

Let $L: \mathcal{S}^{\prime} \rightarrow \mathcal{S}^{\prime}$ be a symmetric, Euclidean invariant linear operator. For reasons which will become transparent in Section 4 , we call an equation of the type $L \xi=$ $\eta, \eta \in \mathcal{S}^{\prime}$, a generalized Poisson equation ${ }^{3}$ (GPE). Suppose that $L$ is continuously invertible $^{4}$ by a Green's function $G \in \mathcal{S}^{\prime}$, i.e. $G * \omega=L^{-1} \omega \forall \omega \in \mathcal{S}^{\prime}$. Then the stochastic GPE

$$
L X=F
$$

has a pathwhise solution $X=G * F$ and $X$ is called a convoluted generalized white noise.

If the Lévy measure $r$ of $F$ has moments of all orders, then the Schwinger functions

$$
S_{n}\left(f_{1} \otimes \cdots \otimes f_{n}\right)=\mathbb{E}_{P^{X}}\left[\prod_{l=1}^{n} X\left(f_{l}\right)\right], \quad f_{1}, \ldots, f_{n} \in \mathcal{S}
$$

exist and can be calculated explicitly. They fulfill the requirements of temperedness, symmetry, invariance, Hermiticity and clustering of the OsterwalderSchrader axioms [49]. In general they do not fulfill the axiom of reflection positivity, cf. [3, 39] for some counter examples (but we also note that the question is not yet completely settled in the general case). Nevertheless, if $G$ has a representation of the form

$$
G=\int_{0}^{\infty} C_{m} d \rho\left(m^{2}\right), \quad \int_{0}^{\infty} \frac{d|\rho|\left(m^{2}\right)}{m^{2}}<\infty,
$$

for some (signed) measure $\rho$ and $C_{m}$ the covariance function of Nelson's free field of mass $m$, then the Schwinger functions (5) can be analytically continued to a sequence of Wightman functions which fulfill all Wightman's axioms 58 except (possibly) for positivity [3]. The Wightman functions however fulfill the Hilbert space structure condition of Morchio and Strocchi [46] and therefore can be considered as vacuum expectation values of a local, relativistic quantum field with indefinite metric [4].

\subsection{Some special Green's functions}

The Green's functions $G=G_{\alpha}$ associated with the partial pseudo differential operators $L_{\alpha}=\left(-\Delta+m_{0}^{2}\right)^{\alpha}, m_{0}>0,0<\alpha \leq 1 / 2$, are of particular interest, since for $F$ a purely Gaussian white noise, $X$ is a generalized free field [36], in particular, $X$ is reflection positive [49, 55] (cf. item (i) below). In the special case $\alpha=1 / 2, X$ is Nelson's free field of mass $m_{0}>0$ [4]. We give a list of the properties of the kernels $G_{\alpha}$ in the following

\footnotetext{
${ }^{3}$ Set $L=-\Delta$ and $\eta$ a signed measure to obtain the Poisson equation of electrostatics.

${ }^{4}$ Here we only deal with GPEs leading to short range static felds.
} 
Proposition 2.1 For $m_{0}>0$ and $\alpha \in(0,1]$ let $G_{\alpha}=G_{\alpha, m_{0}}$ be the Green's function of $\left(-\Delta+m_{0}^{2}\right)^{\alpha}$. Then

(i) $G_{\alpha}$ has a representation (6) with

$$
d \rho_{\alpha}\left(m^{2}\right)=\sin (\pi \alpha) 1_{\left\{m^{2}>m_{0}^{2}\right\}}\left(m^{2}\right) \frac{d m^{2}}{\left(m^{2}-m_{0}^{2}\right)^{\alpha}} \quad 0<\alpha<1
$$

and $\rho_{1}\left(d m^{2}\right)=\delta\left(m^{2}-m_{0}^{2}\right) d m^{2}$;

(ii) $G_{\alpha} \in L^{1}\left(\mathbb{R}^{d}, d x\right)$ and $G_{\alpha}$ is smooth on $\mathbb{R}^{d} \backslash\{0\}$;

(iii) $G_{\alpha}(x)>0 \forall x \in \mathbb{R}^{d} \backslash\{0\}$;

(iv) $\exists C>0$ such that $G_{\alpha}(x) \leq C e^{-m_{0}|x|} \forall x \in \mathbb{R}^{d}:|x|>1$;

(v) For $\lambda>0, G_{\alpha, m_{0}}(\lambda x)=\lambda^{2 \alpha-d} G_{\alpha, \lambda m_{0}}(x)$.

(vi) $\left|G_{\alpha m_{0}}(x)\right|<c_{\alpha}(d)|x|^{-(d-2 \alpha)}$ for $x \in \mathbb{R}^{d} \backslash\{0\}$, where $0<c_{\alpha}(d)<\infty$, for $d \geq 2,0<\alpha<1$, can be chosen optimal as in Eq. (9) below.

Proof. All properties hold for $C_{m_{0}}=G_{1, m_{0}}$, cf. 34 p. 126. The representation (i) has been established in 3], Section 6. (iii) now follows from the fact that $\rho_{\alpha}$ is a positive measure and $C_{m}(x)>0 \forall x \neq 0$. (iv) follows from (i) and the related property of $C_{m}, m \geq m_{0}$. (v) is an consequence of the representation

$$
G_{\alpha, m_{0}}(x)=(2 \pi)^{-d} \int_{\mathbb{R}^{d}} \frac{e^{i k \cdot x}}{\left(|k|^{2}+m_{0}^{2}\right)^{\alpha}} d k
$$

where the integral has to be understood in the sense of Fourier transform of a tempered distribution. (ii) follows from (iv) and (vi); Smoothness of $G_{\alpha}$ for $x \neq 0$ follows from the fact that by (i) $G_{\alpha}$ can be represented as a Fourier-Laplace transform and therefore is real analytic for such $x$. The same argument (using also the "mass-gap" in (i)) also shows that that partial derivatives of $G$ are in $L^{1}\left(\mathbb{R}^{d} \backslash B_{1}(0), d x\right)$.

Finally it remains to prove (vi): Let $\lambda=|x|$ and $\hat{e} \in \mathbb{R}^{d},|\hat{e}|=1$. By rotation invariance of the $G_{\alpha, m_{0}}$ we get using (i), (v) and the residuum theorem

$$
\begin{aligned}
G_{\alpha, m_{0}}(x) & =\lambda^{-(d-2 \alpha)} \sin (\pi \alpha) \int_{\lambda^{2} m_{0}^{2}}^{\infty} C_{m}(\hat{e}) \frac{d m^{2}}{\left(m^{2}-m_{0}^{2}\right)^{\alpha}} \\
& =\lambda^{-(d-2 \alpha)} \gamma_{\alpha}(d) \int_{0}^{\infty} \int_{0}^{\infty} \frac{e^{-\sqrt{t^{2}+m^{2}+\lambda^{2} m_{0}^{2}}}}{\sqrt{t^{2}+m^{2}+\lambda^{2} m_{0}^{2}}} t^{d-2} d t \frac{d m^{2}}{m^{2 \alpha}} \\
& <\lambda^{-(d-2 \alpha)} \gamma_{\alpha}(d) \int_{0}^{\infty} \int_{0}^{\infty} \frac{e^{-\sqrt{t^{2}+m^{2}}}}{\sqrt{t^{2}+m^{2}}} t^{d-2} d t \frac{d m^{2}}{m^{2 \alpha}}
\end{aligned}
$$

We have set $\gamma_{\alpha}(d)=\operatorname{Vol}\left(S^{d-2}\right) \sin (\pi \alpha) / 4 \pi$. Here the right hand side multiplied with $\lambda^{d-2 \alpha}$ defines the constants $c_{\alpha}(d)$ and it is clear from the calculation that these constants are optimal for $\lambda \rightarrow 0$. For $d>2$ it is obvious, that the integrals 
converge. For $d=2,0<\alpha<1$, the inner integral has a logarithmic singularity at $m=0$. This singularity multiplied with $m^{-2 \alpha}$ is however $d m^{2}$-integrable and thus $c_{\alpha}(d)<\infty$ also in this case.

Remark 2.2 (i) As Prop. 2.1(v) shows, $G_{\alpha, 0} \notin L^{1}\left(\mathbb{R}^{d}, d x\right)$ but $G_{\alpha, 0} \in L_{\mathrm{loc}}^{1}\left(\mathbb{R}^{d}, d x\right)$. (ii) For $m_{0}>0, d / 4 \geq \alpha>0$ we have $G_{\alpha} \notin L^{2}\left(\mathbb{R}^{d}, d x\right)$ since $\int_{\mathbb{R}^{d}} G_{\alpha}^{2} d x=$ $G_{2 \alpha}(0)=\infty$, see also Prop. 2.1 (vi).

(iii) For $d=1, \alpha>1 / 4$ we have $G_{\alpha} \in L^{2}\left(\mathbb{R}^{d}, d x\right)$, in particular this applies to $\alpha=1 / 2$.

In particular, we can deduce from Proposition 2.1(i) that the Schwinger functions of the model with $G=G_{\alpha}$ can be analytically continued to Wightman functions, which have been calculated explicitly in [3. From these explicit formulae one can see that for $0<\alpha<1 / 2$ the mass-shell singularities of the truncated Wightman functions are of order $\kappa^{-\alpha}\left(\kappa=k^{0}-\omega_{m_{0}}, \omega_{m}=\left(|\mathbf{k}|^{2}+m^{2}\right)^{1 / 2}\right)$ and hence the model does not describe scattering particles ${ }^{5}$. In the most important case $\alpha=1 / 2$ one can construct incoming and outgoing multi-particle states using the method of 22 but the scattering is trivial, since the mass shell singularities of the Wightman functions in momentum space are of the order $\kappa^{-1 / 2}$ and are thus too weak to produce nontrivial scattering (for that one requires order $\kappa^{-1}$ ). In this sense, the convoluted generalized white noise models can still be considered to be "free fields", even though higher order truncated Wightman functions do not vanish. But it should also be noted that such higher order truncated Wightman functions can be decomposed into a superposition of "structure functions" with non trivial scattering behavior [2].

\subsection{Lattice approximation of noise fields and infinitely divisible laws}

Finally in this section we want to give some heuristic evidence that it is natural to define the generalized white noise $F$ as in (2) and (3): Heuristically speaking, a noise field is a collection of independent identically distributed (i.i.d.) random variables $\{F(x)\}_{x \in \mathbb{R}^{d}}$. To make this notion precise, we substitute the continuum $\mathbb{R}^{d}$ with a lattice $\mathbb{L}_{n}=\frac{1}{n} \mathbb{Z}^{d}, n$ odd, of lattice spacing $1 / n$ and we consider the limit $n \rightarrow \infty$ for i.i.d. random variables $\left\{F_{n}(x)\right\}_{x \in \mathbb{L}_{n}}$. We require that the distribution of the average of the random variables $F_{n}(x)$ remains constant in the unit cube $\Lambda_{1}$ centered at zero, i.e.

$$
F_{1}(0) \stackrel{\mathcal{L}}{=} \sum_{x \in \mathbb{L}_{n} \cap \Lambda_{1}} F_{n}(x) / n^{d} .
$$

We remark that $\sharp \Lambda_{1} \cap \mathbb{L}_{n}=n^{d}$. Eq. (10) can only be fulfilled for $n \in \mathbb{N}$ arbitrary if $F_{1}(0)$ has infinitely divisible probability law and thus by Schoenberg's theorem

\footnotetext{
${ }^{5}$ The use of partial pseudo differential operators leads to mass smearing which in some sense is related to the concept of "infra particles", cf. [54].
} 
[20] $\mathbb{E}\left[e^{i t F_{1}(0)}\right]=e^{\psi(t)}$ for some conditionally positive definite function $\psi$ and $\mathbb{E}\left[e^{i t F_{n}(x) / n^{d}}\right]=e^{\psi(t) / n^{d}}$. Furthermore, (if $\psi$ is $C^{1}$-differentiable) a representation (3) is given by the Lévy-Khintchine theorem [20]. For $f \in \mathcal{S}$ with compact support we set $\left\langle F_{n}, f\right\rangle=\sum_{x \in \mathbb{L}_{n}} F_{n}(x) f(x) / n^{d}$ and we get

$$
\begin{aligned}
\mathbb{E}\left[e^{i\left\langle F_{n}, f\right\rangle}\right] & =\prod_{x \in \mathbb{L}_{n}} \mathbb{E}\left[e^{i F_{n}(x) f(x) / n^{d}}\right] \\
& =\prod_{x \in \mathbb{L}_{n}} e^{\psi(f(x)) / n^{d}} \\
& =e^{\sum_{x \in \mathbb{L}_{n}} \psi(f(x)) / n^{d}} \rightarrow e^{\int_{\mathbb{R}^{d}} \psi(f) d x} \text { as } n \rightarrow \infty
\end{aligned}
$$

where the last step shows that the lattice approximation $F_{n}$ converges to $F$ in law as $n \rightarrow \infty$, cf. (22). For further information on the lattice approximation see [16.

\section{Path properties of convoluted Poisson white noise and ultra-violet finite local interactions}

\subsection{Path properties and quantum field theory}

We say that a random field $X$ realized on the probability space $\left(\mathcal{S}^{\prime}, \mathcal{B}\left(\mathcal{S}^{\prime}\right), P^{X}\right)$ has paths in $E$, where $E \subseteq \mathcal{S}^{\prime}$ is a continuously embedded topological vector space, if $E$ has $P^{X}$ inner measure one, i.e. $\sup _{\mathcal{B}\left(\mathcal{S}^{\prime}\right) \ni B \subseteq E} P^{X}\{X \in B\}=1 .{ }^{6}$ The path properties of $X$ then are given by the general properties of the distributions in $E$, e.g. the property that they can be represented as functions.

The rather irregular paths of Nelson's free field can be considered as the main source of problems in constructive quantum field theory. For $d \geq 2$ the paths are contained in weighted Sobolev spaces with negative index and have no representation as function spaces [22, 51, 61. Consequently, energy densities $v(X)$ needed for the construction of local field interactions are ill-defined. In $d=2$ (and partially also in $d=3$ ) local interactions of polynomial, exponential and trigonometric type have been defined via regularization of paths and application of a renormalization procedute leading to the definition of "Wick-ordered" local interactions : $v(X):$ (for the path properties of these : $v(X):$ see e.g. 61]). Increasing irregularity of the paths as $d \geq 4$ (in physical terms: increasing ultraviolet divergences) so far do not allow an application of these techniques to the physical case $d=4$.

\footnotetext{
${ }^{6}$ Usually one only demands that $\{X \in E\}$ has $P^{X}$ outer measure 1 , but for our considerations we need this stronger formulation.
} 
It is therefore an interesting feature of convoluted Poissson noise (CPN), i.e. a convoluted generalized white noise such that the Lévy characteristic (31) has only a Poisson part ${ }^{7}$, that for a large class of convolution kernels $G$ the paths are given by locally integrable functions and thus some local interactions can be defined without renormalization and therefore give ultra-violet finite interactions. This works independently of the space-time dimension $d \geq 2$ (and, of course, also for $d=1)$.

\subsection{Poisson noise and locally finite marked configurations}

Let us first recall a well-known construction, see e.g. [14]: Let $\Lambda_{n} \subseteq \mathbb{R}^{d}$ be a monotone sequence of compact sets s.t. $\Lambda_{n} \uparrow \mathbb{R}^{d}$ as $n \rightarrow \infty$ and $\Lambda_{0}=\emptyset$. For $n \in \mathbb{N}$ let $D_{n}=\Lambda_{n} \backslash \Lambda_{n-1}$ and we denote the (Lebesgue) volume of $D_{n}$ by $\left|D_{n}\right|$. Let $\left(\left\{N_{n}\right\}_{n \in \mathbb{N}},\left\{Y_{n}^{j}\right\}_{n, j \in \mathbb{N}},\left\{S_{n}^{j}\right\}_{j, n \in \mathbb{N}}\right)$ be three families of independent random variables on some proability space $(\Omega, \mathcal{B}, P)$ which are distributed as follows: $N_{n}: \Omega \rightarrow \mathbb{N}_{0}$ has a Poisson law with intensity $z\left|D_{n}\right|$, i.e. $P\left\{N_{n}=l\right\}=e^{-z\left|D_{n}\right|} z^{l}\left|D_{n}\right|^{l} / l$ !,$Y_{n}^{j}$ : $\Omega \rightarrow \mathbb{R}^{d}$ has uniform distribution on $D_{n}$ (i.e. $1_{D_{n}} d x /\left|D_{n}\right|$ ) and the distribution of $S_{n}^{j}: \Omega \rightarrow \mathbb{R}$ is given by the Lévy measure $r$. From now on we will assume that $r$ has compact support, supp $r \subseteq[-c, c]$, for some $c>0$. By $\mathcal{D}^{\prime}$ we denote the space of (not necessarily tempered) distributions. We define a mapping $\phi: \Omega \rightarrow \mathcal{D}^{\prime}$ via

$$
\phi=\sum_{n=1}^{\infty} \phi_{n}, \quad \phi_{n}=\sum_{j=1}^{N_{n}} S_{n}^{j} \delta_{Y_{n}^{j}}
$$

where $\delta_{x}$ is the Dirac measure in $X$. Obviously, $\phi$ has range in the space of locally finite marked configurations, which is defined as the space of (real) signed measures $\gamma$ on $\mathbb{R}^{d}$ such that $\sharp(\operatorname{supp} \gamma \cap \Lambda)<\infty$ for any compact $\Lambda \subseteq \mathbb{R}^{d}$. By $|\gamma|$ we denote the absolute of the signed measure $\gamma$. Let $f$ be a positive measurable

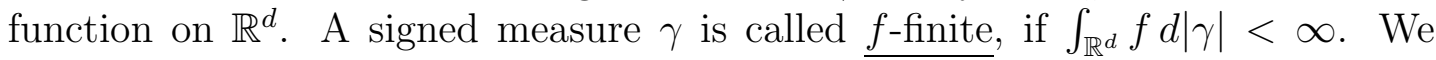
also use the notation $\langle\gamma, f\rangle=\int_{\mathbb{R}^{d}} f d \gamma$ for a (signed) measure $\gamma$ on $\mathbb{R}^{d}$, provided that the integral exists. In particular this is always the case if both $f$ and $\gamma$ are nonnegative.

Proposition 3.1 (i) $\phi$ is $P$-a.s. $f$-finite $\forall f \in L^{1}\left(\mathbb{R}^{d}, d x\right) \cap L^{\infty}\left(\mathbb{R}^{d}, d x\right), f>0$; (ii) In particular, $\phi \in \mathcal{S}^{\prime}$ P-a.s. For $\mathcal{N}$ the exceptional null set, $\phi:(\Omega \backslash \mathcal{N}, \mathcal{B} \cap$ $(\Omega \backslash \mathcal{N})) \rightarrow\left(\mathcal{S}^{\prime}, \mathcal{B}\left(\mathcal{S}^{\prime}\right)\right)$ is measurable;

(iii) Let $F$ be the Poisson white noise with pure Poisson Lévy characteristic determined by $r$ and $z$ and let $P^{F}$ be the associated measure on $\left(\mathcal{S}^{\prime}, \mathcal{B}\left(\mathcal{S}^{\prime}\right)\right)$ s.t. $F$ is the coordinate process w.r.t. $P^{F}$. Then $\phi_{*} P=P^{F}$.

(iv) Assume that $f$ as above is also continuous. Then $F$ has paths in the space of $f$-finite, locally finite marked configurations, that is an element of $\mathcal{B}\left(\mathcal{S}^{\prime}\right)$.

\footnotetext{
${ }^{7}$ With only minor modifications, the considerations of this work can be extended to fields which also have a deterministic part.
} 
The estimates obtained in this proposition actually are not better than those known in the literature. We give a proof for the convenience ofthe reader.

Proof. (i) Since $[-c, c] \times D_{n} \ni(s, y) \rightarrow\left\langle s \delta_{y}, f\right\rangle=s f(y) \in \mathbb{R}$ is measurable, we get that $\left\langle\phi_{n}, f\right\rangle$ and $\left\langle\left|\phi_{n}\right|, f\right\rangle$ are measurable real-valued random variables. Since $\langle|\phi|, f\rangle=\sum_{n=1}^{\infty}\left\langle\left|\phi_{n}\right|, f\right\rangle \in[0, \infty]$ converges by monotonicity, the 1.h.s. of this equation is measurable.

$\mathbb{E}_{P}\left[e^{\langle|\phi|, f\rangle}\right]<\infty$ implies $P\{\langle|\phi|, f\rangle<\infty\}=1$. We can now use the following Laplace transform estimate

$$
\begin{aligned}
\mathbb{E}_{P}\left[e^{\langle|\phi|, f\rangle}\right] & =\lim _{N \rightarrow \infty} \mathbb{E}_{P}\left[e^{\left\langle|\phi|, \Lambda_{\Lambda} f\right\rangle}\right] \\
& =\lim _{N \rightarrow \infty} \prod_{n=1}^{N} \mathbb{E}_{P}\left[e^{\left\langle\left|\phi_{n}\right|, f\right\rangle}\right] \\
& =\lim _{N \rightarrow \infty} \prod_{n=1}^{N}\left[e^{-z\left|D_{n}\right|} \sum_{l=0}^{\infty} \frac{z^{l}\left|D_{n}\right|^{l}}{l !} \int_{D_{n}^{\times l} \times[-c, c] \times l}\right. \\
& \left.\times e^{\sum_{j=1}^{l}\left|s_{n}^{j}\right| f\left(y_{n}^{j}\right)} \frac{d y_{n}^{1}}{\left|D_{n}\right|} \cdots \frac{d y_{n}^{l}}{\left|D_{n}\right|} d r\left(s_{n}^{1}\right) \cdots d r\left(s_{n}^{l}\right)\right] \\
& =\lim _{N \rightarrow \infty} \prod_{n=1}^{N} e^{z \int_{D_{n} \times[-c, c]}\left(e^{|s| f(y)}-1\right) d y d r(s)} \\
& =e^{z \int_{\mathbb{R}^{d}} \int_{[-c, c]}\left(e^{|s| f(y)}-1\right) d r(s) d y} \\
\leq & e^{z \int_{\mathbb{R}^{d}}\left(e^{c f(y)}-1\right) d y} \leq e^{z c\|f\|_{\infty} e^{c\|f\|_{\infty}}\|f\|_{1}}<\infty .
\end{aligned}
$$

Here $\|.\|_{p}$ denotes the norm of $L^{p}\left(\mathbb{R}^{d}, d x\right), p \in[1, \infty]$ and the limits in the intermediate steps always exist by monotonicity.

(ii) follows immediately, since the choosing $f(x)=1 /\left(1+|x|^{2}\right)^{d}$ shows that $|\phi|$, and hence also $\phi$, is polynomially bounded $P$-a.s. . To show measurability of $\phi$, by definition of $\mathcal{B}\left(\mathcal{S}^{\prime}\right)$ it suffices to show that $\langle\phi, f\rangle$ is measurable $\forall f \in \mathcal{S}$ and this can be proven as in (i).

(iii) By a calculation which is analogous to (13) one can show that $\mathcal{C}_{F}(f)=$ $\mathbb{E}_{P}\left[e^{i\langle\phi, f\rangle}\right]=\int_{\mathcal{S}^{\prime}} e^{i\langle\omega, f\rangle} d \phi_{*} P(\omega)$ and the statement follows from the uniqueness of $P^{F}$ which holds by Minlos' theorem.

To show (iv) we first remark that by (iii) the range of $\phi$ is in this set. Thus, the set of $f$-bounded, locally finite marked configurations has $P^{F}$ outer measure one. It remains to show that it is a measurable set. Firstly, the set of locally finite marked configurations $\Gamma$ in $\mathcal{S}^{\prime}$ can be written as

$$
\bigcap_{R \in \mathbb{Q}_{+}} \bigcup_{n \in \mathbb{N}} \bigcap_{\varepsilon \in \mathbb{Q}_{+}} \bigcup_{\substack{s_{1}, \ldots, s_{n} \in \mathbb{Q} \\ y_{1}, \ldots, y_{n} \in \mathbb{Q}^{d}}} \bigcap_{\substack{h \in \tilde{\mathcal{D}}\left(B_{R}\right) \\\|h\|_{\infty}<1}}\left\{\omega \in \mathcal{S}^{\prime}:\left|\omega(h)-\sum_{l=1}^{n} s_{l} h\left(y_{l}\right)\right|<\epsilon\right\}
$$


where $\tilde{\mathcal{D}}\left(B_{j}\right)$ is a countable, dense subset of the set of test functions with support in the ball centered at zero with radius $R$. Thus, $\Gamma$ is measurable. The subset of $f$-finite elements in $\Gamma$ can be written in manifestly measurable form as

$$
\bigcup_{C \in \mathbb{Q}_{+}} \bigcap_{n \in \mathbb{N}} \bigcap_{\substack{h \in \tilde{\mathcal{S}} \\\|h\|_{\infty} \leq 1}}\left\{\omega \in \Gamma:\left|\omega\left(h f_{n}\right)\right|<C\right\}
$$

where $f_{n} \in \mathcal{S}$ is a monotone sequence of positive functions approximating $f$ from below in the local uniform topology and $\tilde{\mathcal{S}}$ is a countable, dense subset of $\mathcal{S}$. This concludes the proof.

By item (iii) of Prop. 3.1 we can identify $\phi$ with $F$ and we therefore drop the notion $\phi$ in the following.

\subsection{Path properties of convoluted Poisson noise}

From the path properties of $F$ we now can deduce the path properties of $X=G * F$ as follows:

Theorem 3.2 Let $F$ be a Poisson noise with Lévy measure $r$ of compact support, supp $r \subseteq[-c, c]$, and let $G \in L^{1}\left(\mathbb{R}^{d}, d x\right)$. Then $X=G * F$ has paths in $L^{1}\left(R^{d}, g_{\epsilon} d x\right)$ where $\epsilon>0$ and $g_{\epsilon}(x)=1 /\left(1+|x|^{2}\right)^{(d+\epsilon) / 2}$.

Proof. Let $\Gamma^{|G| * g_{\epsilon}}$ be the set of $|G| * g_{\epsilon}$-finite, locally finite marked configurations. One can easily check that $|G| * g_{\epsilon}$ fulfills the conditions on $f$ in Proposition 3.1. As proven there, this set is $\mathcal{B}\left(\mathcal{S}^{\prime}\right)$-measurable. By our general assumptions on $L, L^{-1}: \mathcal{S}^{\prime} \rightarrow \mathcal{S}^{\prime}$ is continuous and thus is a measurable transformation on $\left(\mathcal{S}^{\prime}, \mathcal{B}\left(\mathcal{S}^{\prime}\right)\right)$. Since $P^{X}=L_{*} P^{F}=P^{F} \circ L^{-1}$, the support of $P^{X}$ lies in the measurable set $L^{-1} \Gamma^{|G| * g_{\epsilon}}$ and we have to prove that this set lies in $L^{1}\left(\mathbb{R}^{d}, g_{\epsilon} d x\right)$. Let $\Lambda_{n} \uparrow \mathbb{R}^{d}, \Lambda_{n} \subseteq \mathbb{R}^{d}$ open and bounded. Let furthermore $D_{n}^{l}=\Lambda_{n} \backslash \Lambda_{l}$ for $n>l$. For $\gamma \in \Gamma^{|G| * g_{\epsilon}}$, we denote the restriction of $\gamma$ to an open set $A \subseteq \mathbb{R}^{d}$ by $\gamma_{A}$. Clearly, $G * \gamma_{\Lambda_{n}} \in L^{1}\left(\mathbb{R}^{d}, g_{\epsilon} d x\right)$ since $G$ is in $L^{1}\left(\mathbb{R}^{d}, d x\right)$ and supp $\gamma_{\Lambda_{n}}$ is finite. The following estimate shows that $G * \gamma_{\Lambda_{n}}$ forms a Cauchy sequence in $L^{1}\left(\mathbb{R}^{d}, g_{\epsilon} d x\right)$. With $\|\cdot\|_{\epsilon, 1}$ the $L^{1}$-norm on that space, we get

$$
\begin{aligned}
\sup _{n>l}\left\|G * \gamma_{\Lambda_{n}}-G * \gamma_{\Lambda_{l}}\right\|_{\epsilon, 1} & =\sup _{n>l}\left\|G * \gamma_{D_{n}^{l}}\right\|_{\epsilon, 1} \\
& \leq \sup _{n>l} \int_{\mathbb{R}^{d}}|G| * g_{\epsilon} d\left|\gamma_{D_{n}^{l}}\right| \\
& =\int_{\mathbb{R}^{d} \backslash \Lambda_{l}}|G| * g_{\epsilon} d|\gamma| \rightarrow 0 \text { as } l \rightarrow \infty
\end{aligned}
$$

since $\gamma$ is $|G| * g_{\epsilon}$-finite. Also,

$$
\lim _{n \rightarrow \infty}\left\langle G * \gamma_{\Lambda_{n}}, f\right\rangle=\lim _{n \rightarrow \infty}\left\langle\gamma_{\Lambda_{n}}, G * f\right\rangle=\langle\gamma, G * f\rangle=\langle G * \gamma, f\rangle \quad \forall f \in \mathcal{S},
$$


and by the fact that convergence in $L^{1}\left(\mathbb{R}^{d}, g_{\epsilon} d x\right)$ implies convergence in $\mathcal{S}^{\prime}$, we get that $G * \gamma$ coincides with the limit of $G * \gamma_{\Lambda_{n}}$ in the Banach space $L^{1}\left(\mathbb{R}^{d}, g_{\epsilon} d x\right)$.

We remark that by Proposition 2.1 the kernels $G_{\alpha}$ for $0<\alpha \leq 1$ fulfill the requirements of Theorem 3.2 .

In the context of quantum vector fields obtained from SPDEs driven by a Poisson white noise path properties have been considered in [11, 12, 13, 32, 33, 60] where in the latter references it is proven $\mathrm{CPN}$ has piecewise smooth paths with discrete singularities. This has been used to define Wilson loop observables or stochastic co-surfaces (for this concept see [14, 26] and references therein). Local $L^{1}$-integrability of paths does not hold for all of these models, since the Green's functions for vector-valued fields in many case cannot be represented by locally integrable functions. Nevertheless, most of the analysis of this paper would also be possible using the path properties derived in the references given above at the price of more restrictive assumptions on the interactions (to be introduced in the following subsection).

\subsection{Definition of local potentials}

Having established the path properties of the CPN model, we now want to define nonlinear, local interactions. The construction is based on the elementary fact that for a measurable function $v: \mathbb{R} \rightarrow \mathbb{R}$ such that $|v(t)| \leq a+b|t|$ for some $a, b>0$ the nonlinear transformation $L^{1}\left(\mathbb{R}^{d}, g_{\epsilon} d x\right) \ni f \rightarrow v(f) \in L^{1}\left(\mathbb{R}^{d}, g_{\epsilon} d x\right)$ is well defined.

Theorem 3.3 Let $v: \mathbb{R} \rightarrow \mathbb{R}$ be a measurable function s.t. $|v(t)| \leq a+b|t|$ for some $a, b \geq 0$ and let $X$ be a CPN as in Theorem 3.2. Let $\Lambda \subseteq \mathbb{R}^{d}$ compact and $\beta \geq 0$. Then

(i) $v(X)$ is a random field with paths in $L^{1}\left(\mathbb{R}^{d}, g_{\epsilon} d x\right), \epsilon>0$;

(ii) $V_{\Lambda}=\left\langle v(X), 1_{\Lambda}\right\rangle \in \cap_{p \geq 1} L^{p}\left(\mathcal{S}^{\prime}, P^{X}\right)$;

(iii) $e^{-\beta V_{\Lambda}} \in \cap_{p \geq 1} L^{p}\left(\mathcal{S}^{\prime}, P^{X}\right)$;

(iv) Let $\Xi_{\Lambda}=\Xi(z, \beta, \Lambda)=\mathbb{E}_{P^{X}}\left[e^{-\beta V_{\Lambda}}\right]$. Then

$$
P^{\bar{X}_{\Lambda}}=\frac{e^{-\beta V_{\Lambda}}}{\Xi_{\Lambda}} P^{X}
$$

defines a probability measure on $\left(\mathcal{S}^{\prime}, \mathcal{B}\left(\mathcal{S}^{\prime}\right)\right)$.

Proof. (i) That $X \in L^{1}\left(\mathbb{R}^{d}, g_{\epsilon} d x\right) \Rightarrow v(X) \in L^{1}\left(\mathbb{R}^{d}, g_{\epsilon} d x\right)$ is elementary. It remains to prove that $\langle v(X), f\rangle$ is measurable. To this aim let $v$ be continuous and $\chi^{\varepsilon}$ be a sequence of Schwartz functions s.t. $\chi^{\varepsilon} \rightarrow \delta_{0}$ as $\varepsilon \rightarrow 0$. Let $\chi_{x}^{\varepsilon}$ be the translation of $\chi^{\varepsilon}$ by $x$. Then $v\left(X\left(\chi^{\varepsilon, x}\right)\right)$ is a random variable. For a fixed random parameter in the set $G * \Gamma^{|G| * g_{\epsilon}}$ of $P^{X}$ measure one, $X(x)$ is a $L^{1}\left(\mathbb{R}^{d}, g_{\epsilon} d x\right)$ 
function in $x$, cf. the proof of Theorem 3.2. For random parameters in the exceptional null set we re-define $v\left(X\left(\chi_{x}^{\varepsilon}\right)\right)$ to be zero. Approximating the integral by a Riemannian sum, we get that also $\int_{\Lambda} v\left(X\left(\chi_{x}^{\varepsilon}\right)\right) f(x) d x$ is measurable since the pointwise limit of measurable functions is measurable. Since $X$ is a $L^{1}\left(\mathbb{R}^{d}, g_{\epsilon} d x\right)$ function, there exists a subsequence $\varepsilon_{n}$ s.t. in the limit $\varepsilon \rightarrow 0 X\left(\chi^{\varepsilon_{n}, x}\right) \rightarrow X(x)$ $d x$-a.e. and $v\left(X\left(\chi^{\epsilon_{n}, x}\right)\right) \rightarrow v(X(x)) d x$-a.e. for $v$ continous. Consequently, the integral $\int_{\Lambda} v\left(X\left(\chi_{x}^{\varepsilon_{n}}\right)\right) f(x) d x$ converges to $\langle v(X), f\rangle$ by dominated convergence. Thus, this expression is measurable for continuous $v$. By an approximation of a measurable $v$ by continuous functions, using the dominated convergence theorem again, we get that $\langle v(X), f\rangle$ is measurable also for $v$ assumed to be only measurable.

(ii) and (iv) follow from (iii) with $v(t)$ replaced with $-|v(t)|$.

(iii) Since $\left(e^{-\beta V_{\Lambda}}\right)^{p}=e^{-p \beta V_{\Lambda}}$ it suffices to prove the statement for $p=1$. We note that

$$
-\beta V_{\Lambda}=-\beta\left\langle v(X), 1_{\Lambda}\right\rangle \leq \beta b\left\langle|F|,|G| * 1_{\Lambda}\right\rangle+\beta a|\Lambda|
$$

and that $\beta b|G| * 1_{\Lambda} \in L^{1}\left(\mathbb{R}^{d}, d x\right) \cap L^{\infty}\left(\mathbb{R}^{d}, d x\right)$. Thus $\mathbb{E}_{P^{X}}\left[e^{-\beta V_{\Lambda}}\right]<\infty$ follows as in the estimate (13).

Remark 3.4 The growth condition on $v$ in Theorem 3.3 can be relaxed in various ways. E.g. obviously for $v$ positive, (iii) is trivially satisfied and to see that $V_{\Lambda}<\infty P^{X}$-a.s. the condition $v(G) \in L_{\text {loc }}^{1}\left(\mathbb{R}^{d}, d x\right)$ would be sufficient. A refined analysis of this point is postponed to later work (see however the examples of Section 5).

We denote the coordinate process associated to $P^{\bar{X}_{\Lambda}}$ by $\bar{X}_{\Lambda}$ and we call it interacting CPN with infra-red cut-off $\Lambda$.

\section{The connection with particle systems in the grand canonical ensemble}

In this section we explain, how models of $\mathrm{CPN}$ with local interaction can be interpreted as systems of interacting classical, continuous particles in the configurational grand canonical ensemble (GCE).

\subsection{Continuous classical particles in the grand canonical ensemble}

To begin with, we recall some notions of statistical mechanics following [52]. Let $(y, p, s) \in \mathbb{R}^{d} \times \mathbb{R}^{d} \times[-c, c]$ be the "coordinates" of a classical point particle of mass $M>0$ in $d$-dimensional Euclidean space. Here $y$ gives the position, $p$ the momentum and $s$ is an "internal parameter", called charge, which is not 
dynamic, i.e. is not altered by the interaction with other particles. The classical Hamiltonian of $n$ such particles is given by

$$
H\left(y_{1}, \ldots, y_{n} ; p_{1}, \ldots, p_{n} ; s_{1}, \ldots, s_{n}\right)=\sum_{l=1}^{n} \frac{\left|p_{l}\right|^{2}}{2 M}+U\left(y_{1} \ldots, y_{n} ; s_{1}, \ldots, s_{n}\right)
$$

where $U\left(y_{1}, \ldots, y_{n} ; s_{1}, \ldots, s_{n}\right)$ is the potential energy. We assume that there is some a priori distribution of the charges $s$ given by a probability measure $r$ with $\operatorname{supp} r \subseteq[-c, c]$.

The GCE at inverse temperature $\beta>0$ with chemical potential $\mu \in \mathbb{R}$ in the finite volume $\Lambda \subseteq \mathbb{R}^{d}, \Lambda$ compact, is given (up to normalization) by the following measures on the $n$-particle configuration space

$$
\frac{1}{n !} e^{\beta\left[n \mu-H\left(y_{1}, \ldots, y_{n} ; p_{1}, \ldots, p_{n} ; s_{1}, \ldots, s_{n}\right)\right]} d y_{1} \cdots d y_{n} d p_{1} \cdots d p_{n} d r\left(s_{1}\right) \cdots d r\left(s_{n}\right)
$$

where $y_{1}, \ldots, y_{n} \in \Lambda, s_{1}, \ldots, s_{n} \in[-c, c]$. Carrying out the Gaussian integral over the momenta, we pass to the configurational GCE (also abbreviated by GCE in the following) defined (up to normalization) through the following measures on spatial $n$-particles configurations ("marked" by charges $s_{1}, \ldots, s_{n}$ )

$$
\frac{z^{n}}{n !} e^{-\beta U\left(y_{1}, \ldots, y_{n} ; s_{1}, \ldots, s_{n}\right)} d y_{1} \cdots d y_{n} d r\left(s_{1}\right) \cdots d r\left(s_{n}\right)
$$

where

$$
z=e^{\beta \mu}\left(\frac{2 \pi M}{\beta}\right)^{d / 2}>0
$$

is the activity of the system ${ }^{8}$. The functions $e^{-\beta U\left(y_{1}, \ldots, y_{n} ; s_{1}, \ldots, s_{n}\right)}$ are called the Boltzmann weights of the system.

\subsection{Interacting Poisson quantum fields and interacting particle systems}

Identifying $\left(y_{1}, \ldots, y_{n} ; s_{1}, \ldots, s_{n}\right), y_{j} \neq y_{l}, l \neq j$, with $\sum_{l=1}^{n} s_{l} \delta_{y_{l}}$ it is easy to show (by a calculation analogous to Eq. (13) ) that in the case $U \equiv 0$ the measure (22) can be identified with the Poisson noise $F_{\Lambda}=1_{\Lambda} F$ where $F$ is has Lévy measure $r$ and activity $z$. Thus, $F_{\Lambda}$ describes a gas of noninteracting particles in the "box" $\Lambda$, see e.g. [31, [50. We here want to extend this analogy to the interacting models of the preceding section:

- We consider (configurational) GCEs of charged, indistinguishable particles in a finite volume $\Lambda$.

\footnotetext{
${ }^{8}$ By an adaptation of $\mu$ and / or $M$ it is possible to consider $z$ and $\beta$ as independent parameters.
} 
- The charges of the particles give rise to a static field; the field of the unit charge in $y$ is given by the Green's function $G(x-y)$; the static fields penetrates ${ }^{9}$ the "walls" of the "box" $\Lambda$.

- The static field $X$ of a charge configuration $\left(y_{1}, \ldots, y_{n} ; s_{1}, \ldots, s_{n}\right) y_{j} \neq y_{l}$, $l \neq j$, is obtained by superposition from the fields of the single particles and is thus given by $\sum_{l=1}^{n} s_{l} G\left(x-y_{l}\right)$; equivalently the static field is obtained as the solution of the generalized Poisson equation $L X=\eta$ with $\eta=\sum_{l=1}^{n} s_{l} \delta_{y_{l}}$ (Fig. 1).
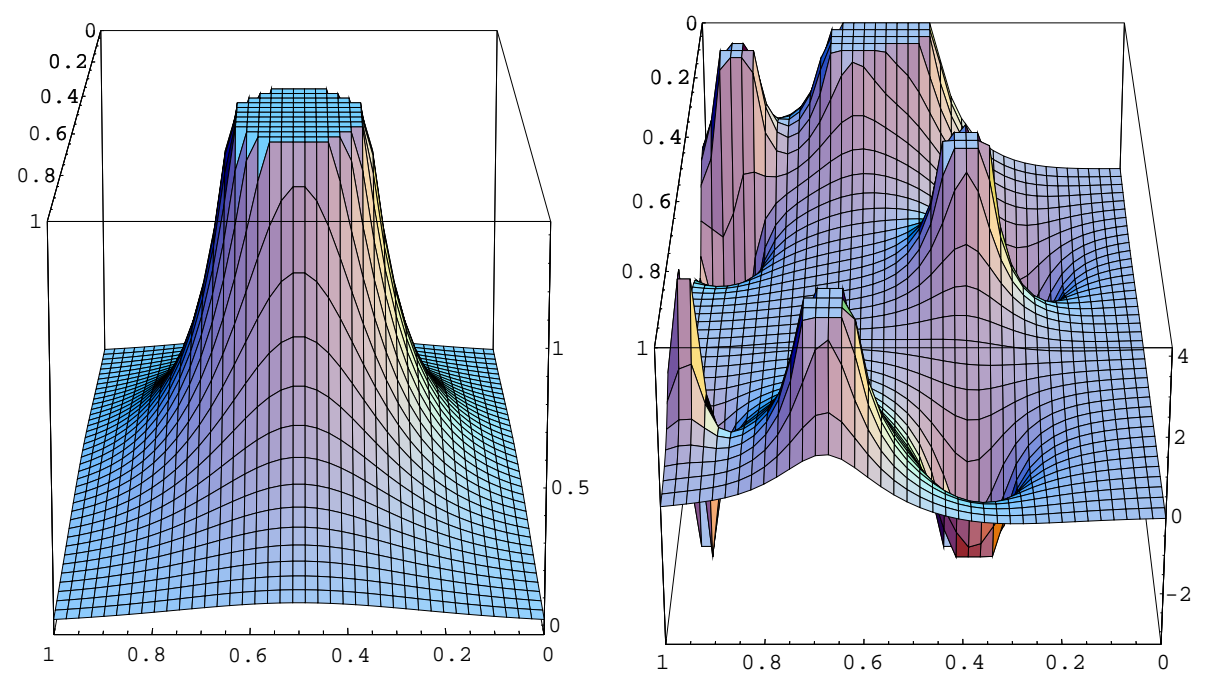

Figure 1: Field of a unit charge and ten particles with positive and negative charges $\pm 1 / \sqrt{10}, G(x)=e^{-m_{0}|x|} /|x|, m_{0}=3$.

- The potential energy of the particle configuration $\eta=\sum_{l=1}^{n} s_{l} \delta_{y_{l}}$ is given by a (nonlinear) energy density $v: \mathbb{R} \rightarrow \mathbb{R}, v(0)=0$, of the static field $X$

$$
\begin{aligned}
U(\eta)=U\left(y_{1}, \ldots, y_{n} ; s_{1}, \ldots, s_{n}\right) & =\int_{\mathbb{R}^{d}} v\left(\sum_{l=1}^{n} s_{l} G\left(x-y_{l}\right)\right) d x \\
& =\int_{\mathbb{R}^{d}} v(G * \eta) d x \\
& =\int_{\mathbb{R}^{d}} v(X) d x
\end{aligned}
$$

The interacting CPN in the finite volume $\Lambda$ is the random field given by the statical field of the interacting particle system in the GCE with potential energy $U$ restricted to the box $\Lambda$.

\footnotetext{
${ }^{9}$ This assumption can be changed by introducing boundary conditions for $L$, cf. Remark 4.2 below.
} 
We remark that the potential $U$ defined in (24) is Euclidean invariant, provided $G$ is invariant under rotations. Furthermore $U$ is symmetric under permutations of arguments $\left(y_{1}, s_{1}\right), \ldots,\left(y_{n} s,_{n}\right)$.

\subsection{Finite volume vs. infra-red cut-off}

Let us now put this into mathematical terms. In particular we want to give sufficient conditions s.t. the potential $U$ in (24) is well-defined and stable.

Let $F_{\Lambda}=1_{\Lambda} F$ be the restriction of $F$ to the compact region $\Lambda$. We set $N_{\Lambda}=\sharp \operatorname{supp} F_{\Lambda}$ and we recall that $N_{\Lambda}$ is Poisson distributed with intensity $z|\Lambda|$. We have $N_{\Lambda}<\infty P^{F}$-a.s. and hence $X_{\Lambda}=G * F_{\Lambda} \in L^{1}\left(\mathbb{R}^{d}, d x\right) P^{F}$-a.s. if $G \in$ $L^{1}\left(\mathbb{R}^{d}, d x\right)$. The crucial observation in (24) is that for the CPN in finite volume $\Lambda, X_{\Lambda}$, we can define local interactions without taking an additional infra-red cut-off as in the usual QFT. Throughout the paper we thus distinguish between the techniques of taking an infra-red cut-off (as in Section 3) and restriction of the associated particle system to a finite volume. While it seems conceptually clear that both formulations lead to the same system if the infra-red cut-off is removed or the infinite volume limit is taken, respectively, this remains to be established mathematically. We now get the counterpart to Theorem 3.3 using a finite volume instead of an infra-red cut-off:

Theorem 4.1 Let $F$ be a Poisson noise and $G$, the Green's function of an operator $L$, as in Theorem 3.2. Let $X_{\Lambda}=G * F_{\Lambda}$. Furthermore, let $v: \mathbb{R} \rightarrow \mathbb{R}$ s.t. $|v(t)| \leq b|t|$ for some $b>0$ and let $\beta>0$. Then

(i) $v\left(X_{\Lambda}\right)$ is a random field with paths in $L^{1}\left(\mathbb{R}^{d}, d x\right)$;

(ii) $\tilde{V}_{\Lambda}=\left\langle v\left(X_{\Lambda}\right), 1_{\mathbb{R}^{d}}\right\rangle \in \cap_{p \geq 1} L^{p}\left(\mathcal{S}^{\prime}, P^{X_{\Lambda}}\right)$ or, equivalently, $U_{\Lambda}=$ $\left\langle v\left(G * F_{\Lambda}\right), 1_{\mathbb{R}^{d}}\right\rangle \in \cap_{p \geq 1} L^{p}\left(\mathcal{S}^{\prime}, P^{F}\right) ;$

(iii) The potential $U_{\Lambda}$ is stable, i.e. for $U_{\Lambda}^{-}$the negative part of $U_{\Lambda}$ we have $U_{\Lambda}^{-} \leq B N_{\Lambda}$ where $B=c b\|G\|_{1}$

(iv) The grand partition function

$$
\tilde{\Xi}_{\Lambda}=\tilde{\Xi}(z, \beta, \Lambda)=\mathbb{E}_{P^{X_{\Lambda}}}\left[e^{-\beta \tilde{V}_{\Lambda}}\right]=\mathbb{E}_{P^{F}}\left[e^{-\beta U_{\Lambda}}\right]
$$

is entire analytic in $z$;

(v) In particular, $e^{-\beta \tilde{V}_{\Lambda}} \in \cap_{p \geq 1} L^{p}\left(\mathcal{S}^{\prime}, P^{X_{\Lambda}}\right)$ or, equivalently, $e^{-\beta U_{\Lambda}} \in$ $\cap_{p \geq 1} L^{p}\left(\mathcal{S}^{\prime}, P^{F}\right)$;

(vi) There exist measures on $\left(\mathcal{S}^{\prime}, \mathcal{B}\left(\mathcal{S}^{\prime}\right)\right)$ defined by

$$
P^{\tilde{X}_{\Lambda}}=\frac{e^{-\beta \tilde{V}_{\Lambda}}}{\tilde{\Xi}_{\Lambda}} P^{X_{\Lambda}}, \quad P^{\tilde{F}_{\Lambda}}=\frac{e^{-\beta U_{\Lambda}}}{\tilde{\Xi}_{\Lambda}} P^{F_{\Lambda}}
$$

related through $L_{*} P^{\tilde{X}_{\Lambda}}=P^{\tilde{F}_{\Lambda}}$. Equivalently, the associated coordinate processes $\tilde{X}_{\Lambda}$ and $\tilde{F}_{\Lambda}$ fulfill the generalized Poisson equation $L \tilde{X}_{\Lambda}=\tilde{F}_{\Lambda} \Leftrightarrow \tilde{X}_{\Lambda}=G * \tilde{F}_{\Lambda}$. 
Proof. (i) That $v\left(X_{\Lambda}\right)$ is a random field can be proven as in Theorem 3.3. That the paths are in $L^{1}\left(\mathbb{R}^{d}, d x\right)$ follows from $\left\langle\left|v\left(X_{\Lambda}\right)\right|, 1_{\mathbb{R}^{d}}\right\rangle \leq b\left\|X_{\Lambda}\right\|_{1}<\infty$.

(ii) follows from (v) with $v$ replaced by $-|v|$. (v) follows from (iv). By [52], Chapter 3, (iv) is a consequence of the stability of the potential (iii).

To prove (iii) we note that

$$
\begin{aligned}
U_{\Lambda}^{-} & \leq\left\langle v^{-}\left(G * F_{\Lambda}\right), 1_{\mathbb{R}^{d}}\right\rangle \\
& \leq b \int_{R^{d}}\left|G * F_{\Lambda}\right| d x \\
& \leq b \int_{R^{d}}|G| *|F| d x=b\|G\|_{1} \int_{\mathbb{R}^{d}} d\left|F_{\Lambda}\right|
\end{aligned}
$$

and $\int_{\mathbb{R}^{d}} d\left|F_{\Lambda}\right| \leq c N_{\Lambda}$. (vi) now follows from (v), the fact that $\tilde{V}_{\Lambda}=U_{\Lambda} \circ L$, cf. Eq. (24), and the transformation formula for probablity measures.

The conditions of Theorem 4.1 on the energy-density $v$ are a little more restrictive than those of Theorem 3.3. where e.g. densities of the form $v(t)=\sqrt{|t|}$ are admissible. In the framework of Theorem 4.1 such potentials can be dealt with at the price of a more technical treatment if one e.g. assumes an exponential decay for $G$, since stability is trivial for positive potentials.

We also point out that in the framework of Theorem 4.1 we can treat the masszero cases (where $G \notin L^{1}\left(\mathbb{R}^{d}, d x\right)$, cf Remark 2.2 ) of Prop. 2.1] if we demand that the (positive) energy density $v$ at $t=0$ tends to zero sufficiently fast, e.g. $0 \leq v(t) \leq c t^{\gamma}$, for $0 \leq|t| \leq \epsilon$, with $\gamma>d /(d-2 \alpha)$.

Remark 4.2 Most of the constructions presented in Section 3 and 4 can be extended to Riemannian manifolds. In particular, we can introduce local interactions on compact manifolds without any cut-off. As a simple example we consider the $d$-dimensional torus $\mathbb{T}_{l}^{d}$ of length $l$ : In this case the Green's functions $G=G_{\alpha, m_{0}}, m_{0}>0$, in Prop. 2.1] have to be modified by introducing periodic boundary conditions for the Laplacian. Then the translation invariant potential $U$ can be defined by $U\left(y_{1}, \ldots, y_{n} ; s_{1}, \ldots, s_{n}\right)=\int_{\mathbb{T}_{l}^{d}} v\left(\sum_{j=1}^{n} s_{j} G\left(x-y_{j}\right)\right) d x$. The proof of stability is completely analogue to the one of Theorem 4.1] The infinite volume limit $\mathbb{T}_{l}^{d} \rightarrow \mathbb{R}^{d}$ now can be studied as $l \rightarrow \infty$.

\section{Models of statistical mechanics seen as 'Pois- son' quantum fields}

In this section we show that a number of well-known particle systems can be associated to an interacting CPN - and hence to a "Poisson", Euclidean QFT - 
in the spirit of Theorem 4.1 (vi). Most of the potentials we discuss in this section do not fulfill directly the requirements of Theorem 4.1. however they are known to fulfill the stability condition (e.g. when the potentials are positive or else by applying well-known criteria, cf. [52]). Hence these potentials (with exception of Section 5.1) can also be used to construct Euclidean quantum field models in our spirit. Moreover they can be obtained by approximation from potentials in the class considered in Theorem 4.1.

\subsection{The gas of hard spheres}

Here we consider a particle system with identical particles carrying a unit charge, hence we set $r=\delta_{1}$, the Dirac measure in 1 . Let $B_{R}=B_{R}(0) \subseteq \mathbb{R}^{d}$ be the open ball centered at zero with radius $R>0$. We set

$$
G(x)=1_{B_{R}}(x)= \begin{cases}1 & \text { if } x \in B_{R} \\ 0 & \text { else }\end{cases}
$$

and we define

$$
v^{\text {h.c. }}(t)= \begin{cases}0 & \text { if } t<2 \\ \infty & \text { if } t \geq 2\end{cases}
$$

Then we get for the potential $U$ in Eq. (24)

$$
\begin{aligned}
U\left(y_{1}, \ldots, y_{n}\right) & =\int_{\mathbb{R}^{d}} v^{\text {h.c. }}\left(\sum_{l=1}^{n} G\left(x-y_{n}\right)\right) d x \\
& = \begin{cases}0 & \text { if } \min _{l, j=1 \ldots, n ; l \neq j}\left|y_{l}-y_{j}\right| \geq R \\
\infty & \text { else }\end{cases}
\end{aligned}
$$

Here we did not write out the arguments $s_{j} \equiv 1$ and the integral in (30) is welldefined as an integral of nonnegative functions with values in $[0, \infty]$. Obviously, on the right hand side of (30) we have the potential of particles with a hard core of radius $R$ ("gas of hard spheres").

We also note that if we modify (29) and set $v(t)=0$ if $t<l$ and $v(t)=\infty$ if $t \geq l, l \in \mathbb{N}, l \geq 2$, we obtain a system where a non empty intersection of $l$ (and more) balls of radius $R$ is energetically forbidden, but all configurations without such intersections have zero potential energy. Such systems have pure $l$-point potentials in the sense of statistical mechanics, cf. [52].

\subsection{Potentials from stochastic geometry}

Here we give "local" formulations of two potentials of stochastic geometry [53, 56, 44, starting with the threshold potential: Let $G$ and $r$ be as in Theorem 4.1 and for $C>0$ we define the energy density

$$
v_{C}(t)= \begin{cases}0 & \text { if } t<C \\ 1 & \text { else }\end{cases}
$$


which obviously is in the class of Theorem 4.1 (set $b=1 / C$ ). We now get (cf. Fig. (2)

$$
\begin{aligned}
U\left(y_{1}, \ldots, y_{n} ; s_{1}, \ldots, s_{n}\right) & =\int_{\mathbb{R}^{d}} v_{C}\left(\sum_{l=1}^{n} s_{l} G\left(x-y_{l}\right)\right) d x \\
& =\left|\left\{x \in \mathbb{R}^{d}: \sum_{l=1}^{n} s_{l} G\left(x-y_{l}\right) \geq C\right\}\right|
\end{aligned}
$$

If we, in particular, choose $G$ and $r$ as in Sect. 5.1, we get the so-called Boolean grain model of stochastic geometry [56]. We can also define similar energy densities $v_{C}^{\text {sym }}(t)=v_{C}(|t|)$ and $v_{-C}=v_{C}^{\text {sym }}-v_{C}$ to obtain related potentials which "threshold" also negative values of $X_{\Lambda}$.

Next we formulate the isodensity contour potential: Let us assume that $G$ is $C^{1}$-differentiable in $\mathbb{R}^{d} \backslash\{0\}$ (cf. Prop. 2.1 (ii) for examples) and $\lim _{x \rightarrow 0}|G(x)|$ $=\infty, \lim _{|x| \rightarrow \infty} G(x)=0$. For $C>0$ we define heuristically

$$
v_{C}^{\text {i.d.c. }}(X)=\delta(X-C)|\nabla X|
$$

or, more precisely,

$$
\begin{aligned}
U\left(y_{1}, \ldots, y_{n} ; s_{1}, \ldots, s_{n}\right) & =\int_{\mathbb{R}^{d}} v_{C}^{\text {i.d.c. }}\left(\sum_{l=1}^{n} s_{l} G\left(x-y_{l}\right)\right) d x \\
& =\lim _{\epsilon \downarrow 0} \epsilon^{-1} \int_{\mathbb{R}^{d}}\left[v_{C-\epsilon}\left(\sum_{l=1}^{n} s_{l} G\left(x-y_{l}\right)\right)\right. \\
& \left.-v_{C}\left(\sum_{l=1}^{n} s_{l} G\left(x-y_{l}\right)\right)\right]\left|\sum_{l=1}^{n} s_{l} \nabla G\left(x-y_{l}\right)\right| d x \\
& =\left|\left\{x \in \mathbb{R}^{d}: \sum_{l=1}^{n} s_{l} G\left(x-y_{l}\right)=C\right\}\right|_{d-1}
\end{aligned}
$$

where $|\cdot|_{d-1}$ denotes the $d$-1-dimensional (surface) volume (cf. Fig 21). Clearly, $\nabla G\left(x-y_{j}\right)$ is well-defined on the set of points where $v_{C-\epsilon}-v_{C}$ does not vanish. The last step follows from the fact that obviously the Hausdorff dimension of the set on the right hand side is $d-1$. This also shows that $U$ is well-defined.

Potentials like $v_{C}$ and $v_{C}^{\text {i.d.c. }}$ might be of particular interest in the continuum limit (see Section 7) since they are designed to measure the fractal properties of the sample paths in that limit, see Fig. [2] 

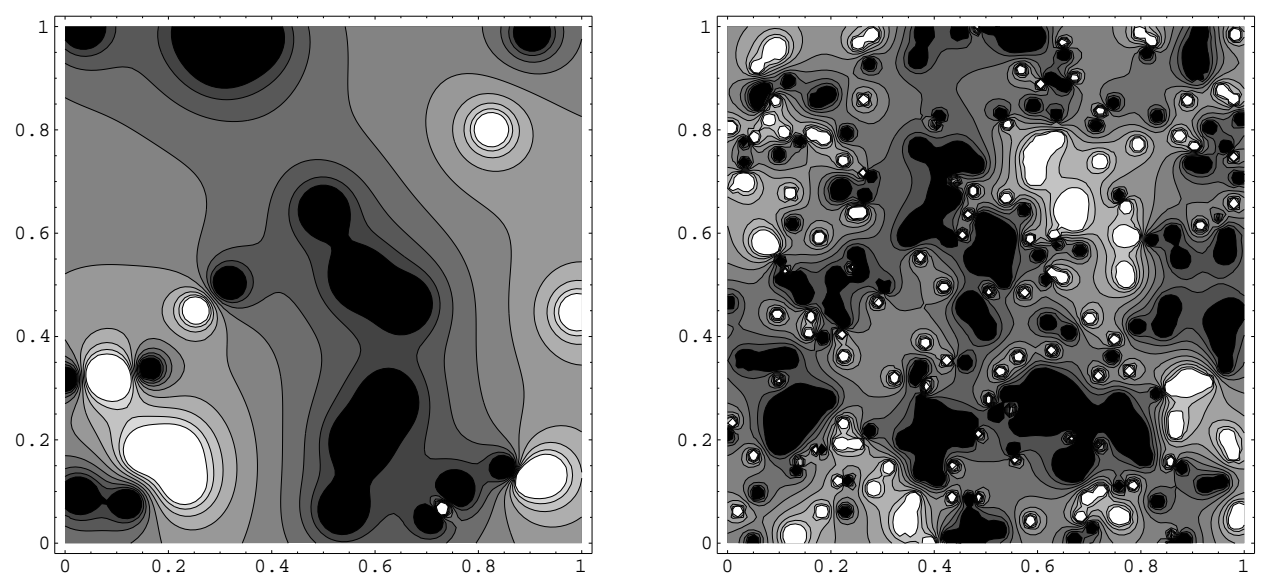

Figure 2: Threshold and isodensity contour potentials for $n=30$ and $n=300$ particles of charge $\pm 1 / \sqrt{n}$ and with $G$ as in Fig. 1. Isodensity contours of integer values from -4 to 4 are displayed. The fractal structure of the continuum limit (Sect. 7) becomes visible.

\subsection{Particle systems with positive definite pair interactions}

Let $r=\delta_{1}$ (in this subsection we may thus omit the variables $s_{l} \equiv 1$ ) and $G$ as in Theorem 4.1 reflection invariant under $x \rightarrow-x$ and let $G$ fulfill $\int_{\mathbb{R}^{d}} G d x \neq 0$. We set $\Phi=G * G$ and we get $\Phi \in L^{1}\left(\mathbb{R}^{d}, d x\right)$. $\Phi$ is positive definite in the sense that $\Phi$ is the Fourier transform of a (not necessarily finite) non negative function on $\mathbb{R}^{d}$. We consider two separate situations: Either $\Phi$ is the Fourier transform of a non negative $L^{1}\left(\mathbb{R}^{d}, d x\right)$-function and hence is continuous. Or we assume that $\Phi$ is nonnegative, in this case possibly $\Phi(0)=\infty$. Also, we remark that choosing $G=G_{\alpha}, 0<\alpha \leq 1 / 2$, as in Proposition 2.1 leads to the second case, cf. Remark 2.2 .

Let $\chi \in C_{0}^{\infty}\left(\mathbb{R}^{d}\right)$ be symmetric, nonnegative such that $\int_{\mathbb{R}^{d}} \chi d x=1$. For $\epsilon>0$, we set $\chi^{\epsilon}(x)=\chi(x / \epsilon) / \epsilon^{d}$ and we introduce an ultra-violet cut-off setting $G^{\epsilon}=\chi^{\epsilon} * G$ and $\Phi^{\epsilon}=G^{\epsilon} * G^{\epsilon}$.

We consider the quadratic energy density $v(t)=t^{2}$ for the ultra-violet regularized model, namely

$$
\begin{aligned}
U_{\epsilon}\left(y_{1}, \ldots, y_{n}\right) & =\int_{\mathbb{R}^{d}}\left(\sum_{l=1}^{n} G^{\epsilon}\left(x-y_{l}\right)\right)^{2} d x \\
& =\sum_{l=1}^{n} \int_{\mathbb{R}^{d}} G^{\epsilon}\left(x-y_{l}\right)^{2} d x+\sum_{\substack{l, j=1 \\
l \neq j}}^{n} \int_{\mathbb{R}^{d}} G^{\epsilon}\left(x-y_{l}\right) G^{\epsilon}\left(x-y_{j}\right) d x \\
& =n \Phi^{\epsilon}(0)+\sum_{\substack{l, j=1 \\
l \neq j}}^{n} \Phi^{\epsilon}\left(y_{l}-y_{j}\right) .
\end{aligned}
$$


If $\Phi(0)=+\infty$, then the first term on the right hand side of (35) in the limit $\epsilon \downarrow 0$ gives an infinite contribution, while the second term remains well-defined (for $\left.y_{l} \neq y_{j}\right)$. Since the first term is proportional to $n$, it can be seen as a (negative) chemical potential or a self-energy which become infinite if $\epsilon \downarrow 0$ - this is very similar to the self-energy problem of a charged point-particle in ordinary electro dynamics. Subtracting this infinite contribution ("self energy renormalization") gives a suitable renormalization for the quadratic potential of the interacting CPN. We want to show that this can be done in a way preserving the local structure of the interaction: We set (note that $\int_{\mathbb{R}^{d}} G^{\epsilon} d x=\int_{\mathbb{R}^{d}} G d x \neq 0$ )

$$
: t^{2}: \text { s.e.r. }=t^{2}-c_{\epsilon} t, \quad c_{\epsilon}=\Phi^{\epsilon}(0) / \int_{\mathbb{R}^{d}} G^{\epsilon} d x
$$

and we get that the renormalized potential

$$
\begin{aligned}
U_{\epsilon}^{\text {s.e.r. }}\left(y_{1}, \ldots, y_{n}\right) & =\int_{\mathbb{R}^{d}}:\left(\sum_{l=1}^{n} G^{\epsilon}\left(x-y_{l}\right)\right)^{2}:_{\epsilon}^{\text {s.e.r. }} d x \\
& =\sum_{\substack{l, j=1 \\
j \neq l}} \Phi^{\epsilon}\left(y_{l}-y_{j}\right)
\end{aligned}
$$

has a well-defined limit as $\epsilon \downarrow 0$, which is just given by the potential resulting from the pair interaction $\Phi$. If $\Phi \geq 0$, stability is obvious. In the case where $\Phi$ is positive definite and continuous, stability follows from Proposition 3.2.7 of [52]. It can be seen in the same reference that positive definite pair potentials play a quite special rôle in the theory of stability.

We would like to point out that a quadratic interaction for a CPN is obviously non-trivial, since a particle gas with pair interactions is obviously different from a gas of noninteracting particles. However, the interaction becomes trivial in the Gaussian (continuum) limit of Section 7 (the interacting process in that limit becomes Gaussian) as can be seen most easily by performing the continuum limit with an ultra-violet cut-off ${ }^{10}$.

\section{High temperature expansion}

In this section we give a construction of the infinite volume limit $\Lambda \uparrow \mathbb{R}^{d}$ (the removal of the infra-red cut-off, respectively) using techniques from continuous particle systems. In particular, we give a high temperature expansion for the

\footnotetext{
${ }^{10}$ The continuum limit for the renormalized potential can be performed setting $r_{1 / \sqrt{z}}=\delta_{1 / \sqrt{z}}$ and $a_{z}=-\sqrt{z}$ in (2) in order to avoid problems with the stability of the potential. This is only slightly different from the techniques in Sect. 7 .
} 
correlation functional for the case of trigonometric interaction. The main trick is to write the trigonometric interaction as effective potential of a (formal) twocomponent marked Potts model at imaginary temperature. Though imaginary temperature might look strange, we show that it does not interfere with the usual cluster expansion method [52. Once the complex valued correlation functional has been constructed for the Potts model, real valuedness of the interaction is restored by restriction to one component. The construction of Gibbs measures then follows from the general analysis of the excellent review article 42, see also the original article by Lennard [43. Obviously, here the techniques are inspired by statistical mechanics of continuous, classical particles. For another construction of infinite volume measures (working also outside the LD-HT regime) with a quantum field flavor that applies to the case where the interaction energy density $v$ is concave and uses FKG inequalities, cf. 37.

\subsection{Trigonometric interactions}

From now on we focus on the case of trigonometric interactions. Let $\nu$ be a

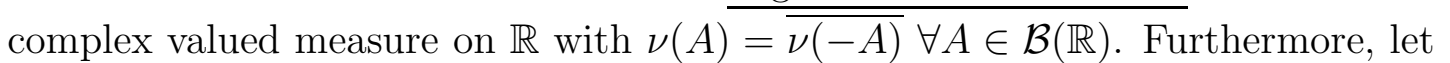
$\nu$ have compact support $\subseteq\left[-c^{\prime}, c^{\prime}\right],|\nu|\left(\left[-c^{\prime}, c^{\prime}\right]\right)<\infty$. Let $b=\int_{\left[-c^{\prime}, c^{\prime}\right]}|\alpha| d|\nu|(\alpha)$ and $b^{\prime}=\int_{[-c, c]}|s| d r(s)$ with $r$ as in equation (3). For the definition of the modulus $|\nu|=|\Re \nu|+|\Im \nu|$ of $\nu$ see e.g. [40]. $\nu$ is called the interaction measure. We set

$$
v(t)=\int_{\mathbb{R}}\left(1-e^{i \alpha t}\right) d \nu(\alpha), t \in \mathbb{R} .
$$

Obviously $v$ is real-valued and fulfills the conditions of Theorem 4.1. Suppose that also $G$ is given as in that theorem.

For $c>0$ let $\Gamma_{0}^{c}\left(\Gamma^{c}\right)$ be the space of signed, real-valued measures $\eta$ on $\mathbb{R}^{d}$ with (locally) finite support such that $-c \leq \eta\{x\} \leq c \forall x \in \mathbb{R}^{d}$. For reasons that are connected with the use of Potts models in the next section, in this section we work with an infra-red cut-off and a finite volume. For $\eta \in \Gamma_{0}^{c}$ and $\Lambda \subset \mathbb{R}^{d}$ compact we thus define the interaction $U_{\Lambda}: \Gamma_{0}^{c} \rightarrow \mathbb{R}$ by $U_{\Lambda}(\eta)=\left\langle v(G * \eta), 1_{\Lambda}\right\rangle$. Furthermore let $F_{\Lambda}$ be a Poisson noise ${ }^{11}$ as in Section 4.3. We define the correlation functional $\rho_{\Lambda}: \Gamma_{0}^{c} \rightarrow(0, \infty)$ associated with $F_{\Lambda}$ and $U_{\Lambda}$ at the inverse temperature $\beta$

$$
\rho_{\Lambda}(\eta)=\frac{1}{\tilde{\Xi}_{\Lambda}} 1_{\{\operatorname{supp} \eta \subseteq \Lambda\}}(\eta) \mathbb{E}_{P^{F}}\left[e^{-\beta U_{\Lambda}\left(\eta+F_{\Lambda}\right)}\right], \forall \eta \in \Gamma_{0}^{c}, \tilde{\Xi}_{\Lambda}=\mathbb{E}_{P^{F}}\left[e^{-\beta U_{\Lambda}\left(F_{\Lambda}\right)}\right] .
$$

What is remarkable is that this correlation functional fulfills the following:

Proposition 6.1 The correlation functional $\rho_{\Lambda}$ fulfills the uniform (in $\Lambda$ ) Ruelle bound $\left|\rho_{\Lambda}(\eta)\right| \leq\left(e^{\beta B}\right)^{\sharp \eta}$ for all $\eta \in \Gamma_{0}^{c}$ with $B=b c\|G\|_{1}$.

\footnotetext{
${ }^{11}$ The associated interacting Poisson noise in this section is denoted by $\tilde{F}_{\Lambda}$.
} 
Proof. As $v$ is differentiable and $\left|v^{\prime}\right|<b$, we get

$$
\begin{aligned}
\left|U_{\Lambda}(\eta+\gamma)-U_{\Lambda}(\gamma)\right| & =\left|\int_{0}^{1} \frac{d}{d t} U_{\Lambda}(\gamma+t \eta) d t\right| \\
& \leq b \int_{\Lambda}|G * \eta| d x \leq b\|G\|_{1} \int_{\Lambda} d|\eta| \leq B \sharp \eta
\end{aligned}
$$

Combining this with the definition of $\rho_{\Lambda}$ in (39) then gives the assertion.

The uniform Ruelle bound is crucial for the passage from infinite volume correlation functionls to Gibbs measures, cf. [42]. In Section 6.4 we come back to this point.

6.2 Two component formal Potts model at imaginary temperature

Let $c^{\prime}>0$ be as in Section 6.1. Clearly $\Lambda^{l} \times \mathbb{R}^{l} \ni\left(y_{1}, \ldots, y_{l} ; s_{1}, \ldots, s_{l}\right) \rightarrow$ $\sum_{j=1}^{l} s_{j} \delta_{y_{j}} \in \Gamma_{0}^{c^{\prime}} \subseteq \mathcal{S}^{\prime}$ is continuous and hence measurable. For $A \in \mathcal{B}\left(\Gamma_{0}\right)$ we thus get that $1_{\left\{\sum_{j=1}^{l} s_{l} \delta_{y_{l}} \in A\right\}}$ is measurable w.r.t. $\mathcal{B}\left(\Lambda^{l}\right) \otimes \mathcal{B}\left(\mathbb{R}^{l}\right)$. We set

$$
\begin{aligned}
P^{Z_{\Lambda}}(A)= & e^{-\beta|\Lambda| \nu\left(\left[-c^{\prime}, c^{\prime}\right]\right)} \delta_{0}(A)+e^{-\beta|\Lambda| \nu\left(\left[-c^{\prime}, c^{\prime}\right]\right)} \sum_{l=1}^{\infty} \frac{\beta^{l}}{l !} \int_{\Lambda^{\times l} \times\left[-c^{\prime}, c^{\prime}\right] \times l} \\
& \times 1_{\left\{\sum_{j=1}^{l} \alpha_{j} \delta_{y_{j}} \in A\right\}}\left(y_{1}, \ldots, y_{l} ; \alpha_{1}, \ldots, \alpha_{l}\right) d y_{1} \cdots d y_{n} d \nu\left(\alpha_{1}\right) \cdots d \nu\left(\alpha_{n}\right) .
\end{aligned}
$$

Here $\delta_{0}(A)=1$ if $0 \in A$ and 0 otherwise.

Lemma 6.2 The function $P^{Z_{\Lambda}}: \mathcal{B}\left(\Gamma_{0}^{c^{\prime}}\right) \rightarrow \mathbb{C}$ is a complex valued measure on $\left(\Gamma_{0}^{c^{\prime}}, \mathcal{B}\left(\Gamma_{0}^{c^{\prime}}\right)\right)$.

Proof. This follows from the fact that $P^{Z_{\Lambda}}$ is a direct sum of such measures.

We note that $P^{Z_{\Lambda}}$ can be seen as a complex valued generalization of a Poisson noise measure. In particular, if $\nu$ is a probability measure, $P^{Z_{\Lambda}}$ is the defining measure for the marked Poisson process in the finite volume $\Lambda$ with mark distribution $\nu$ and intensity $\sigma$.

Let $F_{\Lambda}$ be as in the preceding subsection with associated measure $P^{F_{\Lambda}}$. For $H: \Gamma_{0}^{c} \times \Gamma_{0}^{c^{\prime}} \rightarrow \mathbb{C}$ measurable and $L^{1}\left(P^{F} \otimes\left|P^{Z_{\Lambda}}\right|\right)$ integrable, we define the linear functional

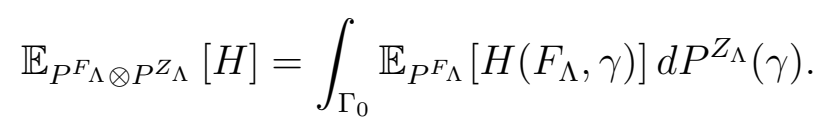

Let $u: \Gamma_{0}^{c} \times \Gamma_{0}^{c^{\prime}} \rightarrow \mathbb{R}$ be defined by $u(\eta, \gamma)=\langle\eta, G * \gamma\rangle$. $u$ is the interaction of a two-component marked Potts model where the component one interacts with the component two but there is no interaction within either component. The 
formal Potts model at imaginary temperature that we consider here is defined by complex valued Gibbs measure in the finite volume

$$
d P^{\text {f.P. }}(\eta, \gamma)=\frac{e^{i u(\eta, \gamma)}}{\mathbb{E}_{P^{F} \otimes P^{Z_{\Lambda}}}\left[e^{i u\left(F_{\Lambda}, Z_{\Lambda}\right)}\right]} d P^{F_{\Lambda}} \otimes P^{Z_{\Lambda}}(\eta, \gamma) .
$$

Here $Z_{\Lambda}(\gamma)=\gamma$ is the coordinate process of the second component. This gives not an ensemble of statistical mechanics (unless $\nu$ is a probability measure), as the second component has a non-probability "distribution" $P^{Z_{\Lambda}}$. Nevertheless, this object can be treated analogously to the measure defining the ordinary Potts model. In particular this applies to the correlation functional

$$
\rho^{\text {f.P. }}(\eta, \gamma)=\frac{1}{\Xi_{\Lambda}^{\text {f.P. }}} 1_{\{\operatorname{supp} \eta \subseteq \Lambda, \operatorname{supp} \gamma \subseteq \Lambda\}}(\eta, \gamma) \mathbb{E}_{P^{F_{\Lambda} \otimes P^{Z_{\Lambda}}}}\left[e^{i u\left(\eta+F_{\Lambda}, \gamma+Z_{\Lambda}\right)}\right],
$$

$\forall \eta \in \Gamma_{0}^{c}, \gamma \in \Gamma_{0}^{c^{\prime}}$ where $\Xi_{\Lambda}^{\text {f.P. }}=\mathbb{E}_{P^{F_{\Lambda} \otimes P^{Z_{\Lambda}}}}\left[e^{i u\left(F_{\Lambda}, Z_{\Lambda}\right)}\right]$. The following crucial observation implies in particular that $\Xi_{\Lambda}^{\text {f.P. }}>0$ :

Proposition 6.3 Let $\tilde{\Xi}_{\Lambda}$ and $\rho_{\Lambda}$ be as in the preceding section. The following identities hold:

(i) $\Xi_{\Lambda}^{\mathrm{f} . \mathrm{P} .}=\tilde{\Xi}_{\Lambda}$;

(ii) $\rho_{\Lambda}^{\text {f.P. }} \cdot(\eta, 0)=\rho_{\Lambda}(\eta)$.

Proof. By the definitions (39) and (43) and Fubini's theorem, it is sufficient to integrate out the second component and show

$$
e^{-\beta U_{\Lambda}(\eta)}=\int_{\Gamma_{0}^{c^{\prime}}} e^{i u(\eta, \gamma)} d P^{Z_{\Lambda}}(\gamma)
$$

with $U_{\Lambda}$ the trigonometric interaction defined in Section 6.1. Using the definition (41) to evaluate the right hand side, we get

$$
\begin{aligned}
& \int_{\Gamma_{0}^{c^{\prime}}} e^{i u(\eta, \gamma)} d P^{Z_{\Lambda}}(\gamma) \\
= & e^{-\beta|\Lambda| \nu\left(\left[-c^{\prime}, c^{\prime}\right]\right)} \sum_{l=0}^{\infty} \frac{\beta^{l}}{l !} \int_{\Lambda^{l} \times\left[-c^{\prime}, c^{\prime}\right] \times l} e^{i\left\langle\eta, G * \sum_{j=0}^{l} \alpha_{j} \delta_{y_{j}}\right\rangle} d y_{1} \cdots d y_{l} d \nu\left(\alpha_{1}\right) \ldots d \nu\left(\alpha_{l}\right) \\
= & e^{-\beta|\Lambda| \nu\left(\left[-c^{\prime}, c^{\prime}\right]\right)} \sum_{l=0}^{\infty} \frac{\beta^{l}}{l !}\left(\int_{\Lambda \times\left[-c^{\prime}, c^{\prime}\right]} e^{i \alpha G * \eta(y)} d \nu(\alpha) d y\right)^{l} \\
= & e^{\beta \int_{\Lambda}\left[\int_{\mathbb{R}} e^{i \alpha G * \eta(y)}-1 d \nu(\alpha)\right] d y}=e^{-\beta \int_{\Lambda} v(G * \eta) d y} .
\end{aligned}
$$


Spelled out in words Proposition 6.3 means that one can obtain the model with trigonometric interaction as the projection (or Widom-Rowlinson model) of a formal Potts model at imaginary temperature. What one has gained from this representation is that the formal Potts model is a model with a pure twopoint interaction, hence the usual cluster expansion procedure of Ruelle goes trough, cf. the following subsection. That the formal Potts model at imaginary temperature for general $\nu$ does not possess the necessary positivity properties poses no problems, as we are only interested in the projection, where positivity holds, cf. Section 6.4.

\subsection{The cluster expansion}

To specify the domain of convergence for our expansion, we define

$$
C_{1}=\sup _{y \in \mathbb{R}^{d}, s \in \text { suppr }} \int_{\mathbb{R}^{d}} \int_{\mathbb{R}}\left|e^{i s \alpha G\left(y-y^{\prime}\right)}-1\right| d|\nu|(\alpha) d y^{\prime} \leq c b\|G\|_{1}<\infty
$$

and

$$
C_{2}=\sup _{y^{\prime} \in \mathbb{R}^{d}, \alpha \in \operatorname{supp} \nu} \int_{\mathbb{R}^{d}} \int_{\mathbb{R}}\left|e^{i s \alpha G\left(y-y^{\prime}\right)}-1\right| d r(\alpha) d y^{\prime} \leq c^{\prime} b^{\prime}\|G\|_{1}<\infty
$$

The following theorem is based on the convergence of Ruelle's cluster expansion [52, Chapter4.4]:

Theorem 6.4 The high-temperature low-density expansion of the infinite volume limit of the correlation functional in the case of trigonometric interactions converges for $z>0$ and $\beta \in \mathbb{R}$ such that $|z|<1 /\left(e C_{1}\right)$ and $|\beta|<1 /\left(e C_{2}\right)$. In particular

(i) $\rho(\eta)=\lim _{\Lambda \uparrow \mathbb{R}^{d}} \rho_{\Lambda}(\eta)$ exists for $\eta \in \Gamma_{0}^{c}$ and depends analytically on $\beta$ and $z$. In particular, for $z$ fixed, the high temperature expansion of $\rho$ converges;

(ii) $\rho$ is invariant under the action of the Euclidean group, i.e. $\rho(\eta)=\rho\left(\eta_{\{g, a\}}\right)$ for $g \in O(d), a \in \mathbb{R}^{d}$;

Proof. (i) To obtain the cluster expansion for $\rho^{\text {f.P. }}=\lim _{\Lambda \uparrow \mathbb{R}^{d}} \rho_{\Lambda}^{\text {f.P. }}$, only a few modifications w.r.t. [52, Chapter 4.4] are necessary.

Let $\mathbb{S}_{a}=\mathbb{R}^{d} \times \mathbb{R} \times\{a\}, a=1,2$ and $\mathbb{S}=\mathbb{S}_{1} \cup \mathbb{S}_{2}=\mathbb{R}^{d} \times \mathbb{R} \times\{1,2\}$. Any pair of finite marked configurations $(\eta, \gamma) \in \Gamma_{0}^{c} \times \Gamma_{0}^{c^{\prime}}$ can then be identified with a non-marked configuration $\xi \in \hat{\Gamma}_{0}(\mathbb{S})$ on $\mathbb{S}$ defined as follows: First, given $\eta=$ $\sum_{j=1}^{n} s_{j} \delta_{x_{j}}$ we define a non-marked configuration $\tilde{\eta}=\sum_{j=1}^{n} \delta_{\left(x_{j}, s_{j}, 1\right)}$ on $\mathbb{S}_{1}$ and likewise $\gamma$ defines a non-marked configuration $\tilde{\gamma}$ on $\mathbb{S}_{2}$. Then we set $\left.\xi\right|_{\mathbb{S}_{1}}=\tilde{\eta}$ and $\left.\xi\right|_{\mathbb{S}_{2}}=\tilde{\gamma}$. Such a non-marked configuration $\xi=\sum_{j=1}^{n} \delta_{q_{j}}, q_{j} \in \mathbb{S}$ can be identified with the finite subset $\left\{q_{1}, \ldots, q_{n}\right\}$ of $\mathbb{S}$. 
Let $\sigma$ be the complex measure on $\mathbb{S}$ obtained by $\left.\sigma\right|_{\mathbb{R}^{d} \times \mathbb{R} \times\{1\}}=d y \otimes r$ and $\left.\sigma\right|_{\mathbb{R}^{d} \times \mathbb{R} \times\{2\}}=d y \otimes \nu$ with $d y$ the Lebsgue measure. For $\Lambda \subseteq \mathbb{R}^{d}$ compact let and $\mathbb{S}_{\Lambda}=\Lambda \times \mathbb{R} \times\{1,2\}$. For $q \in \mathbb{S}$ let furthermore $\zeta(q)=z$ if $q=(y, s, 1), y \in \mathbb{R}^{d}$, $s \in \mathbb{R}$ and $\zeta(q)=\beta$ otherwise.

Let $\chi$ be a function on $\mathbb{S}$ with $\operatorname{supp} \chi \subseteq \mathbb{S}_{\Lambda}$ for some $\Lambda \subseteq \mathbb{R}^{d}$ compact. For $\Psi: \hat{\Gamma}_{0}(\mathbb{S}) \rightarrow \mathbb{C}$ measurable and bounded, we can define

$$
\langle\chi, \Psi\rangle(\zeta)=\Psi_{0}+\sum_{n=1}^{\infty} \frac{1}{n !} \int_{\mathbb{S}^{n}}(\zeta \chi)\left(q_{1}\right) \cdots(\zeta \chi)\left(q_{n}\right) \Psi\left(\left\{q_{1}, \ldots, q_{n}\right\}\right) d \sigma\left(q_{1}\right) \cdots d \sigma\left(q_{n}\right)
$$

Letting $\Psi(\xi)=e^{i u(\eta, \gamma)}$ with $(\eta, \gamma) \in \Gamma_{0}^{c} \times \Gamma_{0}^{c^{\prime}}$ associated with $\xi$, we obtain the following representation of $\rho_{\Lambda}^{\mathrm{f} . \mathrm{P} \cdot:}$

$$
\rho_{\Lambda}^{\mathrm{f} . P .}(\xi)=1_{\left\{\xi \subseteq \mathbb{S}_{\Lambda}\right\}}(\xi)\left\langle\chi_{\Lambda}, \Psi\right\rangle(\zeta)^{-1}\left\langle\chi_{\Lambda}, D_{\xi} \Psi\right\rangle(\zeta), \quad \xi \in \hat{\Gamma}_{0}(\mathbb{S}),
$$

where $D_{\xi} \Psi(\tau)=\Psi(\xi \cup \tau), \xi, \tau \in \hat{\Gamma}_{0}(\mathbb{S})$, and $\chi_{\Lambda}=1_{\mathbb{S}_{\Lambda}}$.

Using Ruelle's *-product (known as $s$-product in QFT [21])

$$
\Psi_{1} * \Psi_{2}(\xi)=\sum_{\tau \subseteq \xi} \Psi_{1}(\tau) \Psi_{2}(\xi \backslash \tau), \quad \xi \in \hat{\Gamma}_{0}(\mathbb{S})
$$

one obtains as in [52, Chapter 4.4] the following expansion of $\rho_{\Lambda}^{\text {f.P. }}$ in the formal parameter $\zeta$

$$
\rho_{\Lambda}^{\text {f.P. }}(\xi)=1_{\left\{\xi \subseteq \mathbb{S}_{\Lambda}\right\}}(\xi)\left\langle\chi_{\Lambda}, \tilde{\varphi}_{\xi}\right\rangle(\zeta), \quad \tilde{\varphi}_{\xi}=\Psi^{-1} * D_{\xi} \Psi .
$$

Here $\Psi^{-1}$ is the inverse of $\Psi$ w.r.t. the $*$-multiplication (as $\Psi(\emptyset)=1$ this inverse exists). We have to study the range of convergence of $\left\langle\chi_{\Lambda}, \tilde{\varphi}_{\xi}\right\rangle(\zeta)$ as $\Lambda \uparrow \mathbb{R}^{d}$. To this aim, we define the pair-potential $o\left(q, q^{\prime}\right)=s s^{\prime} G\left(y-y^{\prime}\right)$ for $q=(y, s, a), q^{\prime}=\left(y^{\prime}, s^{\prime}, a^{\prime}\right)$ with $a^{\prime} \neq a$ and $o\left(q, q^{\prime}\right)=0$ otherwise. For $q \in \xi$ we set $W(q, \xi)=\sum_{q^{\prime} \in \xi \backslash\{q\}} o\left(q, q^{\prime}\right)$ and for $q \in \mathbb{S}, \xi \in \hat{\Gamma}_{0}(\mathbb{S})$ such that $q \notin \xi$, $K(q, \xi)=\prod_{q^{\prime} \in \xi}\left[e^{i o\left(q, q^{\prime}\right)}-1\right]$. As in Ruelle's book, we then obtain the recurrence formula for $\tilde{\varphi}_{\xi}$ and $q \in \xi$

$$
\tilde{\varphi}_{\xi}(\tau)=e^{i W(q, \xi)} \sum_{\kappa \subseteq \tau(q)^{c}} K(q, \kappa) \tilde{\varphi}_{\xi \backslash\{q\} \cup \kappa}(\tau \backslash \kappa),
$$

where $\tau(q)^{c}$ for $q=(y, s, a)$ is defined as $\left\{q^{\prime}=\left(y^{\prime}, s^{\prime}, a^{\prime}\right) \in \tau: a^{\prime} \neq a\right\}$.

One obtains from (48) by induction over $n+m$ with $m=m_{1}+m_{2}=\sharp \xi_{1}+\sharp \xi_{2}$, $\xi_{a}=\xi \cap \mathbb{S}_{a}, a=1,2$, and $n=n_{1}+n_{2}=\sharp \tau_{1}+\sharp \tau_{2}$ that for $\theta_{1}, \theta_{2}>0 \exists C=$ $C\left(\theta_{1}, \theta_{2}\right)<\infty$ such that

$$
\begin{gathered}
\sup _{\substack{\left(q_{1}, \ldots, q_{m}\right) \in \mathbb{S}_{1}^{m_{1}} \times \mathbb{S}_{2}^{m_{j}} q_{j}, q_{l}, j \neq l \\
q_{1}}} \int_{\mathbb{S}_{1}^{n_{1}} \times \mathbb{S}_{2}^{n_{2}}}\left|\tilde{\varphi}_{\left\{q_{1}, \ldots, q_{m}\right\}}\left(\left\{q_{1}^{\prime}, \ldots, q_{n}^{\prime}\right\}\right)\right| d|\sigma|\left(q_{1}^{\prime}\right) \cdots d|\sigma|\left(q_{n}^{\prime}\right) \\
\leq C n_{1} ! n_{2} ! \theta_{1}^{m_{1}} \theta_{2}^{m_{2}}\left(\frac{e^{\theta_{1} C_{1}}}{\theta_{1}}\right)^{n_{1}+m_{1}}\left(\frac{e^{\theta_{2} C_{2}}}{\theta_{2}}\right)^{n_{2}+m_{2}}
\end{gathered}
$$


This estimate for $\theta_{a}=C_{a}^{-1}, a=1,2$, implies that for $m=\sharp \xi$ fixed the right hand side of (47) converges uniformly (in $\Lambda \subseteq \mathbb{R}^{d}$ compact) if

$$
|z|<1 /\left(e C_{1}\right) \text { and }|\beta|<1 /\left(e C_{2}\right)
$$

From the uniform convergence of $\rho_{\Lambda}^{f . P .}(\xi)$ it follows that $\rho^{\text {f.P. }}(\xi)=\lim _{\Lambda \uparrow \mathbb{R}^{d}} \rho_{\Lambda}^{\text {f.P. }}(\xi)$ exists and is analytic in the above parameter domain. Combining this with Proposition 6.3. one obtains the assertion (i) of the theorem.

(ii) Note that $\rho_{\Lambda}\left(\eta_{\{g, a\}}\right)=\rho_{g \Lambda+a}(\eta)$. Invariance of $\rho$ now follows from the equivalence of the limits $\Lambda \uparrow \mathbb{R}^{d}$ and $g \Lambda+a \uparrow \mathbb{R}^{d}$.

Remark 6.5 Let us briefly sketch three methods for an analytic or numerical evaluation of $\rho$. The details can be worked out by (more or less lengthy) straight forward calculations.

(i) Meyer's series for $\rho^{\text {f.P.: }}$ The usual graphical methods connected with the Meyer series [52, p. 88] now can be used for the explicit calculation of the expansion coefficients in $\beta$ and $z$ of $\rho$.

(ii) High temperature expansion: One can go back to Eq. (39) and calculate the $\beta$-expansions of the nominator and the denominator, which essentially amounts to calculating the functional Fourier transforms of $P^{F}$. Taking the quotient in the sense of formal power series then yields an expansion, which is known to converge in the infinite volume limit for $z$ and $\beta$ sufficiently small.

(iii) Small $G$ expansion: Finally, it is possible to adapt 25] to the formal Potts model and to obtain a representation of the moments of the infinite volume Gibbs measure with trigonometric interaction (to be constructed in the following Section) in terms of generalized Feynman graphs ${ }^{12}$, which amounts to a formal expansion in powers of $G$. This method has the advantage that only finitely many moments of $\nu$ appear as parameters in the expansion up to a finite order. Hence the form of the interaction (at least in priciple) can be determined comparing the expansion with experimental data.

\subsection{Construction of Gibbs measures}

Here we want to construct the Gibbs measure associated with the correlation functional $\rho$. Before we can do this, some preparations are needed. Here we mostly follow [42].

The $\sigma$-finite Lebesgue-Poisson measure $\lambda_{z}$ is defined on $\left(\Gamma_{0}^{c}, \mathcal{B}\left(\Gamma_{0}^{c}\right)\right)$ by setting

$$
\lambda_{z}(A)=\delta_{0}(A)+\sum_{l=1}^{\infty} \frac{z^{l}}{l !} \int_{\mathbb{R}^{d l} \times[-c, c] \times l} 1_{A}\left(\sum_{j=1}^{l} s_{j} \delta_{y_{j}}\right) d y_{1} \cdots d y_{l} d r\left(s_{1}\right) \cdots d r\left(s_{l}\right)
$$

\footnotetext{
${ }^{12}$ Here it is necessary that $G$ is a regular function in order to avoid ultra-violet singularities.
} 
$A \in \mathcal{B}\left(\Gamma_{0}^{c}\right)$

It is well-known, see e.g. [42], that $\rho_{\Lambda}$ can be represented as

$$
\rho_{\Lambda}(\eta)=\frac{d P^{\tilde{F}_{\Lambda}}}{d \lambda_{z}}(\eta) \quad \text { for } \lambda_{z}-\text { a.e. } \eta \in \Gamma_{0}^{c}
$$

Here $d P^{\tilde{F}_{\Lambda}}(\eta)=\frac{1}{\tilde{\Xi}_{\Lambda}} e^{-\beta U_{\Lambda}(\eta)} d P^{F_{\Lambda}}(\eta)$.

Let $\Gamma_{\Lambda}^{c}=\left\{\gamma \in \Gamma^{c}: \operatorname{supp} \gamma \subseteq \Lambda\right\}$ for $\Lambda \subseteq \mathbb{R}^{d}$ measurable. Note that for $\Lambda$ compact, $\Gamma_{\Lambda}^{c} \subseteq \Gamma_{0}^{c}$. Suppose $\hat{\rho}: \Gamma_{0}^{c} \rightarrow \mathbb{R}$ is a given functional that fulfills a Ruelle bound $|\hat{\rho}(\eta)| \leq C^{\sharp \eta}$ for some $C>0$. Assume furthermore that the functional is Lennard positive in the sense

$$
q^{\Lambda}(\eta)=\int_{\Gamma_{\Lambda}^{c}}(-1)^{\sharp \eta} \hat{\rho}(\eta+\gamma) d \lambda_{z}(\gamma) \geq 0
$$

for $\lambda_{z}$ - a.e. $\eta$ and all $\Lambda \subseteq \mathbb{R}^{d}$ compact. One can then check that $\left\{P^{\Lambda}\right\}_{\Lambda \subseteq \mathbb{R}^{d} \text { compact }}$ defined by $d P^{\Lambda}(\eta)=q^{\Lambda}(\eta) d \lambda_{z}(\eta)$ is a projective family of probability measures. Hence the inductive limit $P$ of this family exists by Kolmogorov's theorem as a measure on $\left(\Gamma^{c}, \mathcal{B}\left(\Gamma^{c}\right)\right)$, cf. [42, Prop. 4.5 and Theorem 4.5]. In particular this implies that the constructed measure has support on tempered marked configurations.

Furthermore, let $\hat{\rho}_{n}: \Gamma_{0}^{c} \rightarrow \mathbb{R}$ be a sequence of Lennard-positive correlation functionals that fulfill a uniform Ruelle bound $\left|\hat{\rho}_{n}(\eta)\right| \leq C^{\sharp \eta}$ for some $C>0$ independent of $n$. Let furthermore $\hat{\rho}(\eta)=\lim _{n \rightarrow \infty} \hat{\rho}_{n}(\eta)$ exist $\lambda_{z}$-a.e. and suppose that for some $D>0$ sufficiently small $\left\|\hat{\rho}-\hat{\rho}_{n}\right\|_{D} \rightarrow 0$ as $n \rightarrow \infty$ with $\left\|\hat{\rho}-\hat{\rho}_{n}\right\|_{D}=\operatorname{esssup}_{\eta \in \Gamma_{0}^{c}} D^{\sharp \eta}\left|\hat{\rho}(\eta)-\hat{\rho}_{n}(\eta)\right|$. Then the limiting functional $\hat{\rho}$ is Lennard positive and fulfills the same Ruelle bound as the $\hat{\rho}_{n}$ 's, [42, Prop.4.9]. Hence there exists a measure $P$ associated to $\hat{\rho}$, as explained in the preceding paragraph.

Let $p_{\Lambda}: \Gamma_{0}^{c} \rightarrow \Gamma_{\Lambda}^{c}$ be the projection given by $\gamma \rightarrow 1_{\Lambda} \gamma$. A function $H: \Gamma^{c} \rightarrow \mathbb{R}$ is $\Lambda$-measurable, if it is measurable w.r.t. $\mathcal{B}_{\Lambda}\left(\Gamma^{c}\right)=p_{\Lambda}^{-1}\left(\Gamma_{\Lambda}^{c}\right)$. If $\hat{\rho}_{n}$ and $\hat{\rho}$ are as above with associated measures $P$ and $P_{n}$ on $\left(\Gamma^{c}, \mathcal{B}\left(\Gamma^{c}\right)\right)$, then the measures $P_{n}$ converge locally to $P$, i.e. for all $H: \Gamma^{c} \rightarrow \mathbb{R}$ positive that is $\Lambda$-measurable for some $\Lambda \subseteq \mathbb{R}^{d}$ compact, we get $\lim _{n \rightarrow \infty} \mathbb{E}_{P_{n}}[H]=\mathbb{E}_{P}[H]$, see [42, Cor. 4.11].

Also, the translation invariance of $\hat{\rho}$ is equivalent to the the translation invariance of the associated measure, cf. [42, Prop. 3.11].

Applying these pieces of general theory to the case of the preceding subsection, we obtain

Proposition 6.6 Let $P^{\tilde{F}_{\Lambda}}$ be the measures on $\left(\Gamma_{0}, \mathcal{B}\left(\Gamma_{0}\right)\right)$ associated with the correlation functionals $\rho_{\Lambda}$ defined in Section 6.1. Then 
(i) There exists a uniquely determined measure $P^{\tilde{F}}$ on that measurable space which is associated with $\rho=\lim _{\Lambda \uparrow \mathbb{R}^{d}} \rho_{\Lambda}$;

(ii) $\lim _{\Lambda \uparrow \mathbb{R}^{d}} P^{\tilde{F}_{\Lambda}}=P^{\tilde{F}}$ holds in the sense of local convergence;

(iii) $P^{\tilde{F}}$ is translation invariant.

Proof. The estimate (49) implies that $\lim _{\Lambda \uparrow \mathbb{R}^{d}}\left\|\rho_{\Lambda}-\rho\right\|_{D}=0$ for $0<D<z$. The three assertions therefore follow from the general formalism.

Obviously, Proposition 6.6 also implies the existence of $P^{\tilde{X}}=L_{*}^{-1} P^{\tilde{F}}$ as a measure on $\left(\mathcal{S}^{\prime}, \mathcal{B}\left(\mathcal{S}^{\prime}\right)\right)$.

The interaction without infra-red cut-off is $U(\eta)=\int_{\mathbb{R}^{d}} v(G * \eta) d x, \eta \in \Gamma_{0}^{c}$. Let $\eta \in \Gamma_{0}^{c}$ and $\gamma \in \Gamma^{c}$. Then the mutual interaction $W$ between $\eta$ and $\gamma$ is by definition

$$
W(\eta, \gamma)=\int_{\mathbb{R}^{d}}[v(G *(\eta+\gamma))-v(G * \eta)-v(G * \gamma)] d x .
$$

As the derivative of $v$ is bounded, it is easy to see that $W(\eta, \gamma)$ is well-defined and that $|W(\eta, \gamma)|<2 B \sharp \eta$ with $B=b c\|G\|_{1}$ as above.

We say that the measure $P$ on $\left(\Gamma^{c}, \mathcal{B}\left(\Gamma^{c}\right)\right)$ is a Gibbs measure for the interaction $U$, inverse temperature $\beta$ and activity $z$ if for arbitrary $\Lambda \subseteq \mathbb{R}^{d}$ compact and $H: \Gamma^{c} \rightarrow \mathbb{R}$ non-negative the following holds

$$
\mathbb{E}_{P}[H]=\int_{\Gamma_{\Lambda}^{c}} \int_{\Gamma_{\mathbb{R}^{d} \backslash \Lambda}^{c}} H(\eta+\gamma) e^{-\beta U(\eta)-\beta W(\eta, \gamma)} d P(\gamma) d \lambda_{z}(\eta) .
$$

(54) are called Ruelle equations. Under the given conditions they are equivalent with other definitions of Gibbs measures as e.g. Dobrushin-Lanford-Ruelle equations, Georgii-Nguyen-Zessin equations and the standard definition of Gibbs measures via conditional probablilities, cf. [42, Theorem 3.12]. The following theorem verifies the Gibbs property for $P^{\tilde{F}}$.

Theorem 6.7 Let $z, \beta$ as in Theorem 6.4. $G \in L^{1}\left(\mathbb{R}^{d}, d x\right)$. Then $P^{\tilde{F}}$ is a Gibbs measure w.r.t. $U, z, \beta$;

Proof. On both sides of (54) the function $H$ can be approximated from below by elementary functions. Thus, it suffices to consider the case where $H$ is a characteristic function $1_{A}, A \in \mathcal{B}\left(\Gamma_{0}\right)$. On both sides of (53) we have to evaluate $\sigma$-finite measures. It is therefore sufficient to consider $A$ from a $\cap$-stable generating subsystem of $\mathcal{B}\left(\Gamma^{c}\right)$. We may thus assume that $A \in \mathcal{B}_{\Lambda^{\prime}}\left(\Gamma^{c}\right)$ for some $\Lambda^{\prime} \subseteq \mathbb{R}^{d}$ compact.

Let $\Lambda$ be fixed. For compact $\Lambda^{\prime \prime} \subseteq \mathbb{R}^{d}$ we consider the infra-red cut-off interaction $U_{\Lambda^{\prime \prime}}$ as in Section 6.1 and we let $W_{\Lambda^{\prime \prime}}(\eta, \gamma)=U_{\Lambda^{\prime \prime}}(\eta+\gamma)-U_{\Lambda^{\prime \prime}}(\eta)-U_{\Lambda^{\prime \prime}}(\gamma)$, $\eta, \gamma \in \Gamma_{0}^{c}$. 
Using (151) and

$$
\int_{\Gamma_{\tilde{\Lambda}}^{c}} \tilde{H}(\eta) d \lambda_{z}(\eta)=\int_{\Gamma_{\tilde{\Lambda} \backslash \tilde{\Lambda}^{\prime}}^{c}} \int_{\Gamma_{\tilde{\Lambda}^{\prime}}^{c}} \tilde{H}(\eta+\gamma) d \lambda_{z}(\eta) d \lambda_{z}(\gamma)
$$

for compact sets $\tilde{\Lambda}^{\prime} \subseteq \tilde{\Lambda} \subseteq \mathbb{R}^{d}$ and $\tilde{H}: \Gamma^{c} \rightarrow \mathbb{R}$ non-negative and measurable, it is easy to show that the Ruelle equations (54) hold for $P^{\tilde{F}_{\Lambda^{\prime \prime}}}$ instead of $P^{\tilde{F}}$ and $U_{\Lambda^{\prime \prime}}$ and $W_{\Lambda^{\prime \prime}}$ instead of $U$ and $W$.

By Proposition [6.6 (ii) we get that $\lim _{\Lambda^{\prime \prime} \uparrow \mathbb{R}^{d}} \mathbb{E}_{P \tilde{F}_{\Lambda}}[H]=\mathbb{E}_{P \tilde{F}}[H]$. In order to verify the Ruelle equations in the infinite volume limit $\Lambda^{\prime \prime} \uparrow \mathbb{R}^{d}$ one thus has to prove that the r.h.s. of the Ruelle equation with cut-off $\Lambda^{\prime \prime}$ converge to the r.h.s. without that cut-off. The modulus of the difference, which we abbreviate by $I$, can be estimated as follows $\left(\Lambda^{\prime \prime \prime} \subseteq \mathbb{R}^{d}\right.$ is an arbitrary compact set and $\left.\gamma_{\Lambda^{\prime \prime \prime}}=1_{\Lambda^{\prime \prime \prime}} \gamma\right)$ :

$$
\begin{aligned}
I & \leq \mid \int_{\Gamma_{\Lambda}^{c}} \int_{\Gamma_{\mathbb{R}^{d} \backslash \Lambda}^{c}} H(\eta+\gamma) e^{-\beta U(\eta)-\beta W(\eta, \gamma)} d P^{\tilde{F}}(\gamma) d \lambda_{z}(\eta) \\
& -\quad \int_{\Gamma_{\Lambda}^{c}} \int_{\Gamma_{\mathbb{R}^{d} \backslash \Lambda}^{c}} H(\eta+\gamma) e^{-\beta U_{\Lambda^{\prime \prime}}(\eta)-\beta W_{\Lambda^{\prime \prime}}(\eta, \gamma)} d P^{\tilde{F}}(\gamma) d \lambda_{z}(\eta) \mid \\
& +\mid \int_{\Gamma_{\Lambda}^{c}} \int_{\Gamma_{\mathbb{R}^{d} \backslash \Lambda}^{c}} H(\eta+\gamma) e^{-\beta U_{\Lambda^{\prime \prime}}(\eta)-\beta W_{\Lambda^{\prime \prime}}(\eta, \gamma)} d P^{\tilde{F}}(\gamma) d \lambda_{z}(\eta) \\
& -\int_{\Gamma_{\Lambda}^{c}} \int_{\Gamma_{\mathbb{R}^{d} \backslash \Lambda}^{c}} H(\eta+\gamma) e^{-\beta U_{\Lambda^{\prime \prime}}(\eta)-\beta W_{\Lambda^{\prime \prime}}\left(\eta, \gamma_{\Lambda^{\prime \prime \prime}}\right)} d P^{\tilde{F}}(\gamma) d \lambda_{z}(\eta) \mid \\
+ & \mid \int_{\Gamma_{\Lambda}^{c}} \int_{\Gamma_{\mathbb{R}^{d} \backslash \Lambda}^{c}} H(\eta+\gamma) e^{-\beta U_{\Lambda^{\prime \prime}}(\eta)-\beta W_{\Lambda^{\prime \prime}}\left(\eta, \gamma_{\Lambda^{\prime \prime \prime}}\right)} d P^{\tilde{F}}(\gamma) d \lambda_{z}(\eta) \\
& -\int_{\Gamma_{\Lambda}^{c}} \int_{\Gamma_{\mathbb{R}^{d} \backslash \Lambda}^{c}} H(\eta+\gamma) e^{-\beta U_{\Lambda^{\prime \prime}}(\eta)-\beta W_{\Lambda^{\prime \prime}}\left(\eta, \gamma_{\Lambda^{\prime \prime \prime}}\right)} d P^{\tilde{F}_{\Lambda^{\prime \prime}}}(\gamma) d \lambda_{z}(\eta) \mid \\
+ & \mid \int_{\Gamma_{\Lambda}^{c}} \int_{\Gamma_{\mathbb{R}^{d} \backslash \Lambda}^{c}} H(\eta+\gamma) e^{-\beta U_{\Lambda^{\prime \prime}}(\eta)-\beta W_{\Lambda^{\prime \prime}}\left(\eta, \gamma_{\Lambda^{\prime \prime \prime}}\right)} d P^{\tilde{F}_{\Lambda^{\prime \prime}}(\gamma) d \lambda_{z}(\eta)} \\
& -\int_{\Gamma_{\Lambda}^{c}} \int_{\Gamma_{\mathbb{R}^{d} \backslash \Lambda}^{c}} H(\eta+\gamma) e^{-\beta U_{\Lambda^{\prime \prime}}(\eta)-\beta W_{\Lambda^{\prime \prime}}(\eta, \gamma)} d P^{\tilde{F}_{\Lambda^{\prime \prime}}(\gamma) d \lambda_{z}(\eta) \mid} \mid
\end{aligned}
$$

Let us call these terms $I_{1}\left(\Lambda^{\prime \prime}, \Lambda^{\prime \prime \prime}\right), \ldots, I_{4}\left(\Lambda^{\prime \prime}, \Lambda^{\prime \prime \prime}\right)$. Let $\epsilon>0$ be given - we have to show that for $\Lambda^{\prime \prime}$ sufficiently large and a suitable $\Lambda^{\prime \prime \prime}$ we get $I_{j}\left(\Lambda^{\prime \prime}, \Lambda^{\prime \prime \prime}\right)<\epsilon$, $j=1, \ldots, 4$. 
$I_{1}\left(\Lambda^{\prime \prime}, \Lambda^{\prime \prime \prime}\right)$ in fact only depends on $\Lambda^{\prime \prime}$. Note that the integrand in (53) is dominated by $2 b|G| *|\eta| \in L^{1}\left(\mathbb{R}^{d}, d x\right)$, hence by dominated convergence $U_{\Lambda^{\prime \prime}}(\eta) \rightarrow$ $U(\eta)$ and $W_{\Lambda^{\prime \prime}}(\eta, \gamma) \rightarrow W(\eta, \gamma)$ for $\eta \in \Gamma_{0}^{c}$ and $\gamma \in \Gamma^{c}$. At the same time $e^{2 \beta B \sharp \eta}$ is an upper bound for $\left|H(\eta+\gamma) e^{-\beta U_{\Lambda^{\prime \prime}}(\eta)-\beta W_{\Lambda^{\prime \prime}}(\eta, \gamma)}\right|$, if $H$ is bounded by one. Consequently, $I_{1}\left(\Lambda^{\prime \prime}, \Lambda^{\prime \prime \prime}\right) \rightarrow 0$ as $\Lambda^{\prime \prime} \uparrow \mathbb{R}^{d}$ holds by dominated convergence.

For $\Lambda^{\prime \prime \prime}$ fixed, $I_{3}\left(\Lambda^{\prime \prime}, \Lambda^{\prime \prime \prime}\right) \rightarrow 0$ as $\Lambda^{\prime \prime} \uparrow \mathbb{R}^{d}$ follows from Proposition [6.6 (ii).

It remains to show that one can find a compact set $\Lambda^{\prime \prime \prime}$ such that $I_{2}\left(\Lambda^{\prime \prime}, \Lambda^{\prime \prime \prime}\right)$, $I_{4}\left(\Lambda^{\prime \prime}, \Lambda^{\prime \prime \prime}\right)<\epsilon$ for all compact $\Lambda^{\prime \prime} \subseteq \mathbb{R}^{d}$. Note that this is trivial for $G$ of finite range $R$ and $d\left(\Lambda, \partial \Lambda^{\prime \prime \prime}\right)=\inf \left\{|x-y|: x \in \Lambda, y \in \mathbb{R} \backslash \Lambda^{\prime \prime \prime}\right\}>2 R$ since then $W_{\Lambda^{\prime \prime}}\left(\eta, \gamma_{\Lambda^{\prime \prime \prime}}\right)=W_{\Lambda^{\prime \prime}}(\eta, \gamma)$ implies $I_{2}\left(\Lambda^{\prime \prime}, \Lambda^{\prime \prime \prime}\right)=I_{4}\left(\Lambda^{\prime \prime}, \Lambda^{\prime \prime \prime}\right)=0$.

In the next step we consider $I_{4}\left(\Lambda^{\prime \prime}, \Lambda^{\prime \prime \prime}\right)$ in the general case. Let for $R>0$ $\Lambda_{R}=\left\{x \in \mathbb{R}^{d}: \exists y \in \Lambda\right.$ s.t. $\left.|x-y| \leq R\right\}$. We set $\Lambda^{\prime \prime \prime}=\Lambda_{R^{\prime}}$ for some $R^{\prime}>0$ and we have to show that $I_{4}\left(\Lambda^{\prime \prime}, \Lambda_{R^{\prime}}\right) \rightarrow 0$ uniformly in $\Lambda^{\prime \prime}$ as $R^{\prime} \rightarrow \infty$. Let us begin with the estimate

$$
\begin{aligned}
& \left|e^{-\beta W_{\Lambda^{\prime \prime}}(\eta, \gamma)}-e^{-\beta W_{\Lambda^{\prime \prime}}\left(\eta, \gamma_{\Lambda_{R^{\prime}}}\right)}\right| \\
\leq & e^{2|\beta| B \sharp \eta}\left(e^{|\beta|\left|W_{\Lambda^{\prime \prime}}(\eta, \gamma)-W_{\Lambda^{\prime \prime}}\left(\eta, \gamma_{\Lambda_{R^{\prime}}}\right)\right|}-1\right) .
\end{aligned}
$$

For $R>0$ arbitrary, we can combine (56) with

$$
\begin{aligned}
& \left|W_{\Lambda^{\prime \prime}}(\eta, \gamma)-W_{\Lambda^{\prime \prime}}\left(\eta, \gamma_{\Lambda_{R^{\prime}}}\right)\right| \\
& \leq 2 b\left[\int_{\Lambda_{R}}|G| *\left|\gamma_{\mathbb{R}^{d} \backslash \Lambda_{R^{\prime}}}\right| d x+\int_{\mathbb{R}^{d} \backslash \Lambda_{R}}|G| *|\eta| d x\right] \\
& =2 b\left[\left\langle|G| * 1_{\Lambda_{R}} \cdot 1_{\mathbb{R}^{d} \backslash \Lambda_{R^{\prime}}}|\gamma|\right\rangle+\left\langle|G| * 1_{\mathbb{R}^{d} \backslash \Lambda_{R}},|\eta|\right\rangle\right]
\end{aligned}
$$

and we obtain

$$
\begin{aligned}
& \int_{\Gamma_{\mathbb{R}^{d} \backslash \Lambda}^{c}}\left|e^{-\beta W_{\Lambda^{\prime \prime}}(\eta, \gamma)}-e^{-\beta W_{\Lambda^{\prime \prime}}\left(\eta, \gamma_{\Lambda_{R^{\prime}}}\right)}\right| d P^{\tilde{F}_{\Lambda^{\prime \prime}}(\gamma)} \\
& \quad \leq e^{2 B|\beta| \sharp \eta}\left(e^{2 b|\beta|\left\langle|G| * 1_{\mathbb{R}^{d} \backslash \Lambda_{R}},|\eta|\right\rangle} \int_{\Gamma^{c}} e^{2 b|\beta|\left\langle|G| * 1_{\Lambda_{R}} \cdot 1_{\mathbb{R}^{d} \backslash \backslash \Lambda_{R^{\prime}}},|\gamma|\right\rangle} d P^{\left.\tilde{F}_{\Lambda^{\prime \prime}}(\gamma)-1\right)}\right.
\end{aligned}
$$

For a function $h: \mathbb{R}^{d} \times[-c, c] \rightarrow \mathbb{R}$ we define the Lebsgue-Poisson coherent state $e_{\lambda}(h, \xi)=\prod_{l=1}^{n} h\left(x_{l}, s_{l}\right), \xi=\sum_{j=1}^{n} s_{l} \delta_{x_{l}} \in \Gamma_{0}^{c}$. The following identity holds for $\tilde{H}: \Gamma_{\tilde{\Lambda}}^{c} \rightarrow \mathbb{R}$ non-negative and $\tilde{\Lambda} \subseteq \mathbb{R}^{d}$ an arbitrary measurable set

$$
\int_{\Gamma_{\bar{\Lambda}}^{c}} \int_{\Gamma_{\bar{\Lambda}}^{c}} \tilde{H}(\eta+\gamma) e_{\lambda}\left(h_{1}, \eta\right) e_{\lambda}\left(h_{2}, \gamma\right) d \lambda_{z}(\eta) d \lambda_{z}(\gamma)=\int_{\Gamma_{\tilde{\Lambda}}^{c}} \tilde{H}(\eta) e_{\lambda}\left(h_{1}+h_{2}, \eta\right) d \lambda_{z}(\eta)
$$


and can be found in [42, Corollary 2.5] for $\Gamma_{\tilde{\Lambda}}^{c}$ replaced with $\Gamma_{0}^{c}$. One can check the above identity along the same lines, cf. the proof of Lemma 2.1 of that reference. Using the notation $h(x, s)=|s||G| * 1_{\Lambda_{R}}(x) 1_{\mathbb{R}^{d} \backslash \Lambda_{R^{\prime}}}(x)$ we thus get

$$
\begin{aligned}
& 1 \leq \int_{\Gamma^{c}} e^{2 b|\beta|\left\langle|G| * 1_{\Lambda_{R}} \cdot 1_{\mathbb{R}^{d} \backslash \Lambda_{R^{\prime}}}|\gamma|\right\rangle} d P^{\tilde{F}_{\Lambda^{\prime \prime}}(\gamma)} \\
& =\int_{\Gamma_{\Lambda^{\prime \prime}}^{c}} \int_{\Gamma_{\Lambda^{\prime \prime}}} e_{\lambda}\left(e^{2 b|\beta| h}, \gamma\right) e_{\lambda}(-1, \xi) \rho_{\Lambda^{\prime \prime}}(\gamma+\xi) d \lambda_{z}(\xi) d \lambda_{z}(\gamma) \\
& =\int_{\Gamma_{\Lambda^{\prime \prime}}^{c}} e_{\lambda}\left(e^{2 b|\beta| h}-1, \gamma\right) \rho_{\Lambda^{\prime \prime}}(\gamma) d \lambda_{z}(\gamma) \\
& \leq \int_{\Gamma_{\Lambda^{\prime \prime}}^{c}} e_{\lambda}\left(e^{2 b|\beta| h}-1, \gamma\right) C^{\sharp \gamma} d \lambda_{z}(\gamma) \\
& \leq e^{z C \int_{\Lambda^{\prime \prime}}\left[e^{2 b|\beta| h(x, s)}-1\right] d r(s) d x} \leq e^{2 z C b c|\beta| e^{2 B|\beta|} \int_{\mathbb{R}^{d} \backslash \Lambda_{R^{\prime}}}|G| * 1_{\Lambda_{R}} d x},
\end{aligned}
$$

with $B=c b\|G\|_{1} \geq \sup _{x \in \mathbb{R}^{d}, s \in[-c, c]} h(x, s)$ and $C$ the Ruelle constant that does not depend on $\Lambda^{\prime \prime}$, cf. Prop. 6.1] Inserting (60) into (58) we obtain for $I_{4}\left(\Lambda^{\prime \prime}, \Lambda_{R^{\prime}}\right)$

$$
\begin{aligned}
& I_{4}\left(\Lambda^{\prime \prime}, \Lambda_{R^{\prime}}\right)
\end{aligned}
$$

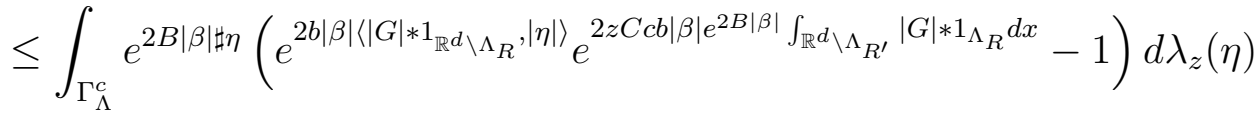

$$
\begin{aligned}
& \leq e^{2 z B|\beta||\Lambda|}\left(e^{2 z C c b|\beta| e^{2 B|\beta|} \int_{\mathbb{R}^{d} \backslash \Lambda_{R^{\prime}}}|G| * 1_{\Lambda_{R}} d x+2 z c b|\beta| \int_{\Lambda}|G| * 1_{\mathbb{R}^{d} \backslash \Lambda_{R}} d x}-1\right) \\
& =e^{2 z B|\beta||\Lambda|}\left(e^{2 z b c|\beta|\left(C e^{2 B|\beta|} \int_{\mathbb{R}^{d} \backslash \Lambda_{R^{\prime}}}|G| * \Lambda_{\Lambda_{R}} d x+\int_{\mathbb{R}^{d} \backslash \Lambda_{R}}|G| * 1_{\Lambda} d x\right)}-1\right) .
\end{aligned}
$$

Let $\epsilon^{\prime}>0$ be arbitrary. We have to show that we can choose $R, R^{\prime}>0$ such that each of the integrals in the exponent on the r.h.s. of (61) is smaller than $\epsilon^{\prime}$. We note that $|G| * 1_{\Lambda} \in L^{1}\left(\mathbb{R}^{d}, d x\right)$, thus $\int_{\mathbb{R}^{d} \backslash \Lambda_{R}}|G| * 1_{\Lambda} d x<\epsilon^{\prime}$ for $R=R\left(\epsilon^{\prime}\right)>0$ large enough. Let such $R$ be fixed, we then see that $|G| * 1_{\Lambda_{R}} \in L^{1}\left(\mathbb{R}^{d}, d x\right)$, hence we can find an $R^{\prime}=R^{\prime}\left(R, \epsilon^{\prime}\right)>0$ large enough, such that $\int_{\mathbb{R}^{d} \backslash \Lambda_{R^{\prime}}}|G| * 1_{\Lambda_{R}} d x<\epsilon^{\prime}$. Choosing $\epsilon^{\prime}=\epsilon^{\prime}(\epsilon)>0$ small enough and $R, R^{\prime}$ accordingly, we can finally achieve that the right hand side of (61) becomes smaller than $\epsilon$, which establishes the required estimate for $I\left(\Lambda^{\prime \prime}, \Lambda_{R^{\prime}}\right)$.

To estimate $I_{2}\left(\Lambda^{\prime \prime}, \Lambda_{R^{\prime}}\right)$, we remark that (57) is independent of $\Lambda^{\prime \prime}$. Thus one obtains (58) with $\tilde{F}_{\Lambda^{\prime \prime}}$ replaced by $\tilde{F}$. The integral over $\Gamma^{c}$ in the first line of (60) with $\tilde{F}_{\Lambda^{\prime \prime}}$ replaced by $\tilde{F}$ fulfills the same uniform bound as on the right hand side, as we have by monotone convergence and Prop 6.6 (ii)

$$
\int_{\Gamma^{c}} e^{2 b \beta\left\langle|G| * \Lambda_{\Lambda_{R}} \cdot 1_{\mathbb{R}^{d} \backslash \Lambda_{R^{\prime}}}\right|\langle\mid\rangle} d P^{\tilde{F}}(\gamma)
$$




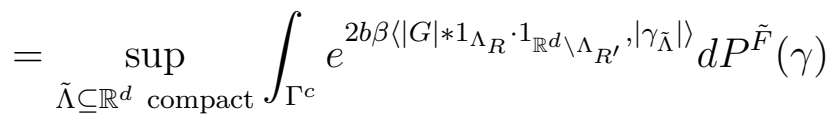

$$
\begin{aligned}
& \leq \sup _{\tilde{\Lambda}, \Lambda^{\prime \prime} \subseteq \mathbb{R}^{d} \text { compact }} \int_{\Gamma^{c}} e^{2 b \beta\left\langle|G| * 1_{\Lambda_{R}} \cdot 1_{\mathbb{R}^{d} \backslash \Lambda \Lambda_{R^{\prime}}}\left|\gamma_{\tilde{\Lambda}}\right|\right\rangle} d P^{\tilde{F}_{\Lambda^{\prime \prime}}(\gamma)}
\end{aligned}
$$

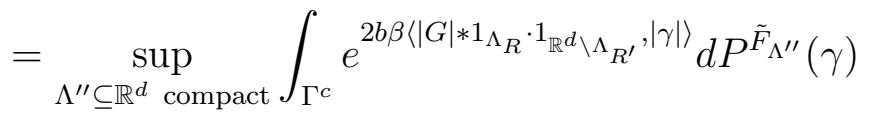

Hence the estimate (61) also holds for $I_{2}\left(\Lambda^{\prime \prime}, \Lambda_{R^{\prime}}\right)$.

6.5 Cluster property and extremality of the state

The aim of this subsection is to show that $P^{\tilde{F}}$ is a pure or extremal Gibbs state, i.e. $P^{\tilde{F}}$ cannot be written as the convex combination of two translation invariant measures on $\left(\Gamma^{c}, \mathcal{B}\left(\Gamma^{c}\right)\right)$.

We first prove a cluster property for the correlation functional $\rho$.

Let $h: \mathbb{R}^{d n} \times[-c, c]^{n} \rightarrow \mathbb{R}$ and $\hat{\rho}: \Gamma_{0}^{c} \rightarrow \mathbb{R}$ a correlation functional. We define $\hat{\rho}(h)=\int_{\mathbb{R}^{d n} \times[-c, c] \times n} \hat{\rho}\left(\sum_{j=1}^{n} s_{j} \delta_{y_{j}}\right) h\left(y_{1}, \ldots, y_{n}, s_{1}, \ldots, s_{n}\right) d y_{1} \cdots d y_{n} d r\left(s_{1}\right) \cdots d r\left(s_{n}\right)$

and $h_{\{g, a\}}\left(y_{1}, \ldots, y_{n}, s_{1}, \ldots, s_{n}\right)=h\left(g y_{1}+a, \ldots, g y_{n}+a, s_{1}, \ldots, s_{n}\right) g \in O(d)$, $a \in \mathbb{R}^{d}$. For $\eta=\sum_{j=1}^{n} s_{j} \delta_{y_{j}} \in \Gamma_{0}^{c}, \eta_{\{g, a\}}=\sum_{j=1}^{n} s_{j} \delta_{g^{-1}\left(y_{j}-a\right)}$.

Proposition 6.8 Let $z, \beta$ as in Theorem 6.4. Then $\rho$ fulfills the cluster property

$$
\lim _{\tilde{\Lambda} \uparrow \mathbb{R}^{d}} \frac{1}{|\tilde{\Lambda}|} \int_{\tilde{\Lambda}}\left[\rho\left(f \otimes h_{\{1, a\}}\right)-\rho(f) \rho(h)\right] d a=0
$$

for $h: \mathbb{R}^{d n_{2}} \times[-c, c]^{n_{2}} \rightarrow \mathbb{R}, f: \mathbb{R}^{d n_{2}} \times[-c, c]^{n_{2}} \rightarrow \mathbb{R}$ infinitely differentiable, bounded and decreasing like a Schwartz test function in all $\mathbb{R}^{d}$-arguments, $n_{1}, n_{2} \in$ $\mathbb{N}$.

Proof. Again, we closely follow [52, Section 4.4.7]. Let $\log ^{*}$ be the logarithm w.r.t. the $*$ product (46) and let $\rho^{T}=\log ^{*} \rho: \Gamma_{0}^{c} \rightarrow \mathbb{R}$ be the cluster functional associated with $\rho$. Let $\varphi=\log ^{*} \Psi$, with $\Psi$ as in the proof of Theorem 6.4 then the following representation holds:

$$
\rho^{T}(\eta)=\sum_{n=0}^{\infty} \frac{1}{n !} \int_{\mathbb{S}^{n}} \zeta\left(q_{1}\right) \cdots \zeta\left(q_{n}\right) D_{(\eta, 0)} \varphi\left(\left\{q_{1}, \ldots, q_{n}\right\}\right) d \sigma\left(q_{1}\right) \cdots d \sigma\left(q_{n}\right) .
$$

And from (49) one obtains for $m=n_{1}+n_{2}, q_{1}=(y, s, 1)$ in combination with $\tilde{\varphi}_{\{q\}}=\varphi_{\{q\}}$, cf. [52, Eq. (4.24)],

$$
\begin{array}{r}
\int_{\mathbb{S}_{1}^{n_{1}-1} \times S_{2}^{n_{2}}}\left|\varphi\left(\left\{q_{1}, \ldots, q_{n}\right\}\right)\right| d|\sigma|\left(q_{2}\right) \ldots d|\sigma|\left(q_{n}\right) \\
\leq C\left(n_{1}-1\right) ! n_{2} ! C_{1}^{-1}\left(e C_{1}\right)^{n_{1}}\left(e C_{2}\right)^{n_{2}}
\end{array}
$$


This finally gives the estimate

$$
\begin{aligned}
& \int_{\mathbb{R}^{d(m-1)} \times[-c, c] \times(m-1)}\left|\rho^{T}\left(\sum_{j=1}^{n} s_{j} \delta_{y_{j}}\right)\right| d y_{2} \cdots d y_{m} d r\left(s_{2}\right) \cdots d r\left(s_{m}\right) \\
& \leq C(m-1) ! C_{1}^{-1} \frac{\left(e C_{1}\right)^{m}}{1-|z| e C_{1}} \frac{1}{1-|\beta| e C_{2}}
\end{aligned}
$$

By a simple change of variables $\int_{\mathbb{R}^{d}}\left|\rho^{T}\left(f \otimes h_{\{1, a\}}\right)\right| d a<\infty$ follows for $f, h$ as in the assertion, which implies (cf. [52, Section 4.4.3]) $\int_{\mathbb{R}^{d}}\left|\rho\left(f \otimes h_{\{1, a\}}\right)-\rho(f) \rho(h)\right| d a<$ $\infty$.

One way to link the cluster property of the correlation functional to ergodicity of the measure is to express the moments of the measure in terms of the correlation functional. This at the same time gives us a formula for the infinite volume Schwinger functions of the associated random field $\tilde{X}$.

Proposition 6.9 Let $z, \beta$ as in Theorem 6.4. Then

(i) All moments of $P^{\tilde{F}}$ exist. In terms of the correlation functional $\rho$ they are given by

$$
\mathbb{E}_{P^{\tilde{F}}}\left[\prod_{j=1}^{l} \tilde{F}\left(f_{j}\right)\right]=\sum_{\substack{\left\{I_{1}, \ldots, I_{j}\right\}: 1 \leq j \leq l, I_{r} \subseteq\{1, \ldots, l\} \\ I_{1} \cup \ldots \cup I_{j}=\{1, \ldots, l\}}} \rho\left(\mathbf{f}_{I}\right)
$$

where for $\mathbf{f}=\left(f_{1}, \ldots, f_{l}\right) \in \mathcal{S}^{\times l}$ and $I=\left(I_{1}, \ldots, I_{j}\right)$ as in the sum in (66) $\mathbf{f}_{I}\left(y_{1}, \ldots, y_{j}, s_{1}, \ldots, s_{j}\right)=\prod_{q=1}^{j} s_{q}^{\sharp I_{q}} \prod_{p \in I_{q}} f_{p}\left(y_{q}\right)$.

(ii) The Schwinger functions of the associated interacting CPN $\tilde{X}$ exist and are are given by $S_{n}\left(f_{1} \otimes \cdots \otimes f_{l}\right)=\mathbb{E}_{P \tilde{X}}\left[\prod_{j=1}^{l} X\left(f_{j}\right)\right]=\mathbb{E}_{P \tilde{F}}\left[\prod_{j=1}^{l} \tilde{F}\left(G * f_{j}\right)\right]$. Furthermore, the Schwinger functions are analytic in the coupling constant (inverse temperature) $\beta$ and the Feynman series converges on the indicated domain.

Proof. (i) From the formula for the local densities $q^{\Lambda}$ and (59), one obtains the following formula for the Laplace transform of $P^{\tilde{F}}$

$$
\mathbb{E}_{P^{\tilde{F}}}\left[e^{\langle f, \tilde{F}\rangle}\right]=\int_{\Gamma_{\Lambda}^{c}} e_{\lambda}\left(e^{f}-1, \eta\right) \rho(\eta) d \lambda_{z}(\eta)
$$

where $f \in \mathcal{D}\left(\mathbb{R}^{d}\right)$ with supp $f \subseteq \Lambda$ and $e_{\lambda}$ is the Lebesgue-Poisson coherent state defined in the proof of Theorem 6.4. In (66) we can replace $\Gamma_{\Lambda}^{c}$ with $\Gamma_{0}^{c}$. From the Ruelle-bound for $\rho$ it follows that the r.h.s. is well-defined not only for $f$ with compact support, but also for $f \in \mathcal{S}$ and hence (66) extends by continuity. Existence of the (two sided) Laplace-transform implies existence of moments of all orders. Taking derivatives of the Laplace-transform at zero yields

$$
\mathbb{E}_{P \tilde{F}}\left[\prod_{j=1}^{l} \tilde{F}\left(f_{j}\right)\right]=\sum_{j=1}^{l} \frac{1}{l !} \sum_{\substack{\left(I_{1}, \ldots, I_{j}\right): I_{q} \subseteq\{1, \ldots, l\} \\ I_{1} \cup \ldots \cup I_{j}=\{1, \ldots, l\}}} \rho\left(\mathbf{f}_{I}\right)
$$


This gives (66) is by symmetry of $\rho$.

(ii) This is a immediate corollary from (i) and $\tilde{X}=G * \tilde{F}$. The analyticity and the range of convergence follows from the related statements for $\rho$.

Combination of Propositions 6.8 and 6.9 gives us

Theorem 6.10 $P^{\tilde{F}}$ is an extremal Gibbs measure.

Proof. Extremality of $P^{\tilde{F}}$ is equivalent to the ergodicity property

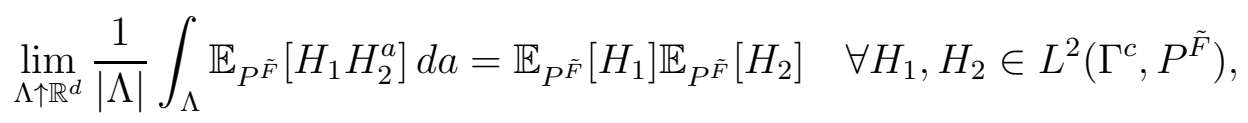

where $H_{2}^{a}(\eta)=H_{2}\left(\eta_{\{1, a\}}\right), \eta \in \Gamma^{c}$, cf. e.g. [17, Section 3.2]. By approximation of both sides of (68) it is furthermore easy to see that it suffices to check (68) for $H_{1}$ and $H_{2}$ in a set that has dense algebraic span in $L^{2}\left(\Gamma^{c}, P^{\tilde{F}}\right)$. Since the twosided Laplace-transform exists for $P^{\widetilde{F}}$, functions of the form $H_{p}=\prod_{j=1}^{l_{p}} \tilde{F}\left(f_{j}^{p}\right)$, $p=1,2$, with $f_{l}^{p} \in \mathcal{S}$ form such a set. On can thus use the cluster property of $\rho$, cf. Theorem 6.4 and (66) as follows:

We note that the right hand side of (66) is a sum over all partitions of $\{1, \ldots, l\}$ into disjoint sets. By (66) (see also [52, Section 4.4.3]) it is therfore sufficient to check that for $\mathbf{f}^{a}=\left(f_{1}^{1}, \ldots, f_{l_{1}}^{1}, f_{1,\{1, a\}}^{2}, \ldots, f_{l_{2},\{1, a\}}^{2}\right)$ and $\mathbf{f}^{p}=\left(f_{1}^{p}, \ldots, f_{l_{p}}^{p}\right)$, $p=1,2$ we have $\lim _{\Lambda \uparrow \mathbb{R}^{d}} \frac{1}{|\Lambda|} \int_{\Lambda} \rho\left(\mathbf{f}_{I}^{a}\right) d a=0$ if $I=\left\{I_{1}, \ldots, I_{j}\right\}$ s.t. $\exists I_{q} \in I$ with $I_{q} \cap\left\{1, \ldots, l_{1}\right\} \neq \emptyset$ and $I_{q} \cap\left\{l_{1}+1, \ldots, l_{1}+l_{2}\right\} \neq \emptyset$ and $\lim _{\Lambda \uparrow \mathbb{R}^{d}} \frac{1}{|\Lambda|} \int_{\Lambda} \rho\left(\mathbf{f}_{I}^{a}\right) d a=$ $\rho\left(\mathbf{f}_{I^{1}}^{1}\right) \rho\left(\mathbf{f}_{I^{2}}^{2}\right)$ with $I^{1}=\left\{I_{q} \in I: I_{q} \subseteq\left\{1, \ldots, l_{1}\right\}\right\}, I^{2}=\left\{I_{q}-l_{1}: I_{q} \subseteq\left\{l_{1}+\right.\right.$ $\left.\left.1, \ldots, l_{1}+l_{2}\right\}\right\}$ otherwise.

The second condition is just the cluster property of $\rho$, cf. Proposition 6.8 To verify the first condition, one can look into the definition of $\mathbf{f}_{I}^{a}$ and $\rho\left(\mathbf{f}_{I}^{a}\right)$ to see (using also Ruelle bounds) that $\rho\left(\mathbf{f}_{I}^{a}\right) \rightarrow 0$ faster than any inverse power of $|a|$, which implies the first condition. In fact, in that case at least one (non-translated) $f_{j}^{1}$ and one (translated) $f_{l,\{1, a\}}^{2}$ are evalated w.r.t. he same integration variable. The product of these two functions thus decreases rapidly, if $a$ gets large.

\subsection{An alternative construction using 'duality'}

Here we prove an equality between correlation functionals of interacting particles systems and characteristic functionals ${ }^{13}$ of interacting CPNs making it possible to apply the results on the infinite volume for the correlation functional from Section 6.3 to the removal of the infra-red cut-off for the characteristic functional. The results obtained here are somewhat weaker than those obtained by the detour

\footnotetext{
${ }^{13}$ We are grateful to an anonymous referee for pointing out to us that the 'duality transformation' discussed here has already been considered by V. Shkripnik from Kiev in some unpublished preprints in the 1970ies.
} 
through correlation functionals, as in the previous section. But still we hope that this new way of performing the thermodynamic limit for the measure is of independent interest, as it e.g. gives a direct perturbative control over the characteristic function.

We restrict to trigonometric interactions given by energy densities $v(t)=$ $\int_{\left[-c^{\prime}, c^{\prime}\right]}\left(1-e^{i \alpha t}\right) d \nu(\alpha)$ where $\nu$ is a symmetric probability measure on $\left[-c^{\prime}, c^{\prime}\right]$ for some $c^{\prime}>0$. Furthermore, in this subsection we also assume that the Lévy measure $r$ is symmetric. Clearly, in that case the formal Potts model of Section 6.2 becomes a real Potts model (however still at imaginary temperature) and one expects a specific symmetry or 'duality' depending on the question, whether the Potts model is projected to its first or its second component.

The following technical lemma states that there is a pointwise definition $X(x)$ of the convoluted Poisson noise $X$ and that also the trigonometric interactions $v(X(x))$ have a pointwise meaning:

Lemma 6.11 Let $X_{\Lambda}=G * F_{\Lambda}$ be a CPN with assumptions on $G$ and $F_{\Lambda}$ as above. For $p \geq 1$, the mapping $\mathbb{R}^{d} \times \mathbb{R} \ni(x, \alpha) \rightarrow e^{i \alpha X_{\Lambda}(x)} \in L^{p}\left(\mathcal{S}^{\prime}, P^{X_{\Lambda}}\right)$ is continuous.

Proof. We prove that $e^{i \alpha X_{\Lambda}(x)}$ is well-defined for $(x, \alpha) \in \mathbb{R}^{d} \times \mathbb{R}$. Continuity can be proven in an analogous manner. Let $X_{\Lambda, \epsilon}=\chi_{\epsilon} * X_{\Lambda}$ be a ultra-violet regularization of $X_{\Lambda}$, cf. Section 5.3. Without loss of generality we restrict ourselves to the case $p=2 n, n \in \mathbb{N}$. Then, for $\epsilon, \epsilon^{\prime}>0$,

$$
\begin{aligned}
& \mathbb{E}_{P^{X_{\Lambda}}}\left[\left|e^{i \alpha X_{\Lambda, \epsilon}(x)}-e^{i \alpha X_{\Lambda, \epsilon^{\prime}}(x)}\right|^{p}\right] \\
= & \mathbb{E}_{P^{X_{\Lambda}}}\left[\left(e^{i \alpha X_{\Lambda, \epsilon}(x)}-e^{i \alpha X_{\Lambda, \epsilon^{\prime}}(x)}\right)^{n}\left(e^{-i \alpha X_{\Lambda, \epsilon}(x)}-e^{-i \alpha X_{\Lambda, \epsilon^{\prime}}(x)}\right)^{n}\right] \\
= & \sum_{j, l=0}^{n}\left(\begin{array}{c}
n \\
j
\end{array}\right)\left(\begin{array}{c}
n \\
l
\end{array}\right)(-1)^{l+j} \mathbb{E}_{P^{X_{\Lambda}}}\left[e^{i \alpha\left[(n-l) X_{\Lambda, \epsilon}(x)+l X_{\Lambda, \epsilon^{\prime}}(x)-(n-j) X_{\Lambda, \epsilon}(x)-j X_{\Lambda, \epsilon^{\prime}}(x)\right]}\right] \\
= & \sum_{j, l=0}^{n}\left(\begin{array}{c}
n \\
j
\end{array}\right)\left(\begin{array}{l}
n \\
l
\end{array}\right)(-1)^{l+j} e^{\int_{\Lambda-x} \psi\left(\alpha(j-l) G *\left(\chi_{\epsilon}-\chi_{\epsilon^{\prime}}\right)(y)\right) d y}
\end{aligned}
$$

We note that $\sum_{j, l=0}^{n}\left(\begin{array}{c}n \\ j\end{array}\right)\left(\begin{array}{c}n \\ j\end{array}\right)(-1)^{l+j}=\left((1-1)^{n}\right)^{2}=0$. In order to prove that $e^{i \alpha X_{\Lambda, \epsilon}(x)}$ forms a Cauchy sequence in $L^{p}\left(\mathcal{S}^{\prime}, P^{X_{\Lambda}}\right)$ it is thus sufficient to show that the integrals on the right hand side of (69) vanish as $\epsilon, \epsilon^{\prime} \downarrow 0$. Clearly, $\left|\int_{\Lambda-x} \psi\left(\alpha(j-l) G *\left(\chi_{\epsilon}-\chi_{\epsilon^{\prime}}\right)(y)\right) d y\right| \leq z c n|\alpha| \int_{\mathbb{R}^{d}}\left|G * \chi_{\epsilon}-G * \chi_{\epsilon^{\prime}}\right| d y$ and now the assertion of the lemma follows from $G * \chi_{\epsilon} \rightarrow G$ in $L^{1}\left(\mathbb{R}^{d}, d x\right)$ as $\epsilon \downarrow 0$ (the latter again is a consequence of the Riesz convergence theorem [18]).

In particular, Lemma 6.11 shows that the characteristic functional $\mathcal{C}_{X}$ of $X$ can be extended to the space $\Gamma_{0}=\cup_{c>0} \Gamma_{0}^{c}$ of finite configurations. Since the 


\begin{tabular}{||l|l|l||}
\hline \hline & $\bar{X}_{\Lambda}$ interacting CPN & $\tilde{F}_{\Lambda}$ interacting particle system \\
\hline 1. & activity $z$ & inverse temperature $\beta$ \\
\hline 2. & inv. temperature (coupling const.) $\beta$ & activity $z$ \\
\hline 3. & IR cut-off and finite volume $\Lambda$ & finite volume and IR-cut off $\Lambda$ \\
\hline 4. & Lévy measure $r$ & interaction measure $\nu$ \\
\hline 5. & interaction measure $\nu$ & Lévy measure $r$ \\
\hline 6. & integral kernel $G$ & integral kernel $G$ \\
\hline \hline
\end{tabular}

Table 1: Identifications for the characteristic-correlation functional duality between 'Poisson' quantum fields and interacting particle systems for the case of trigonometric interactions

measure for the interacting $\mathrm{CPN} \bar{X}, P^{\bar{X}_{\Lambda}}$, is absolutely continuous w.r.t. $P^{X}$, the same holds for the characteristic functional $\mathcal{C}_{\bar{X}_{\Lambda}}$. Let us now recall all the data entering in the definition of $\bar{X}_{\Lambda}$ (cf. the left row of Table 1). We say that an interacting particle system $\tilde{F}_{\Lambda}$ is dual ${ }^{14}$ to the interacting CPN $\bar{X}_{\Lambda}$ if the defining data for $\tilde{F}_{\Lambda}$ can be obtained from the defining data of $\bar{X}_{\Lambda}$ according to Table 1 . The following theorem clarifies the sense of this notion:

Theorem 6.12 Let $\bar{X}_{\Lambda}$ be an interacting $C P N$ with a trigonometric interaction and let $\tilde{F}_{\Lambda}$ be dual to $\bar{X}_{\Lambda}$. Then, the characteristic functional of $\bar{X}_{\Lambda}$ and the correlation functional of $\tilde{F}_{\Lambda}$ are related via $\mathcal{C}_{\bar{X}_{\Lambda}}(\eta)=\rho_{\Lambda}(\eta) \forall \eta \in \Gamma_{0}$, supp $\eta \subseteq \Lambda$.

Proof. By Lemma 6.11 and the estimate $\left|V_{\Lambda}\right|<2|\Lambda|$ we get that all expressions in the following chain of equations are well-defined:

$$
\begin{aligned}
\mathcal{C}_{\bar{X}_{\Lambda}}(\eta) & =\frac{1}{\Xi_{\Lambda}} \mathbb{E}_{P X}\left[e^{i X(\eta)} e^{-\beta V_{\Lambda}}\right] \\
& =\frac{e^{-\beta|\Lambda|}}{\Xi_{\Lambda}} \sum_{n=0}^{\infty} \frac{(-\beta)^{n}}{n !} \mathbb{E}_{P^{X}}\left[e^{i X(\eta)}\left(V_{\Lambda}-|\Lambda|\right)^{n}\right] \\
& =\frac{e^{-\beta|\Lambda|}}{\Xi_{\Lambda}} \sum_{n=0}^{\infty} \frac{\beta^{n}}{n !} \int_{\Lambda^{n} \times[-b, b] \times n} \\
& \times e^{-z \int_{\mathbb{R}^{d}} \int_{[-c, c]}\left[1-e^{i s\left(G * \eta(y)+\alpha_{1} G\left(y-y_{1}\right)+\cdots+\alpha_{n} G\left(y-y_{n}\right)\right)}\right] d r(s) d y} \\
& \times d y_{1} \cdots d y_{n} d \nu\left(\alpha_{1}\right) \cdots d \nu\left(\alpha_{n}\right)
\end{aligned}
$$

Defining $U_{\Lambda}(\eta)=\int_{\Lambda} v_{r}(G * \eta) d x$ with $v_{r}(t)=\int_{[-c, c]}\left(1-e^{i t s}\right) d r(s)$ we now get the statement of the theorem comparing the right hand side of (170) and the defining

\footnotetext{
${ }^{14}$ There is a conceptual difference between this notion of "duality" and the notion of $\tilde{F}_{\Lambda}$ being associated to $\tilde{X}_{\Lambda}$.
} 
equation (39), cf. also (13). That here the partition function $\Xi_{\Lambda}$ of the interacting CPN is equal to the partition function $\tilde{\Xi}_{\Lambda}$ can be seen by an analogous argument.

Theorem 6.12 generalizes the well-known connection of trigonometric interactions and particle systems with certain pair interactions (equivalence of massive / massless sine-Gordon model and Yukawa / Coulomb gas, respectively), see e.g. [9, 10, 27] and references therein. In fact, in the ultra-violet regularized case one can obtain this classical 'duality' from Theorem 6.10 by a scaling in the spirit of Corollary [7.5 below, see also Section 7.4.

From Theorem 6.12 we get that for interacting CPNs with negative trigonometric interactions the high-temperature ('Feynman') expansion of the characteristic functional is equivalent to the low activity expansion of the dual particle system and vice versa. Hence the results of Section 6.3 carry over to characteristic functionals of the fields in the following way:

Corollary 6.13 Let $\beta$ and $z$ be the coupling constant and the activity of the interacting $C P N \tilde{X}_{\Lambda}$. If $|\beta|<1 /\left(e C_{1}\right)$ and $|z|<1 /\left(e C_{2}\right)$ with $C_{1}, C_{2}$ as in (44) and (45). Then

(i) $\mathcal{C}_{\tilde{X}}(\eta)=\lim _{\Lambda \uparrow \mathbb{R}^{d}} \mathcal{C}_{\tilde{X}_{\lambda}}(\eta)$ exists for $\eta \in \Gamma_{0}$ and is analytic in $z$ and $\beta$;

(ii) $\mathcal{C}_{\tilde{X}}(\eta)$ is continuous at zero in the sense that $\mathcal{C}_{\tilde{X}}\left(\sum_{l=1}^{n} \alpha_{l} \delta_{x_{l}}\right) \rightarrow 1$ if $\alpha_{1}, \ldots, \alpha_{l}$ $\rightarrow 0$;

(iii) $\mathcal{C}_{\tilde{X}}: \Gamma_{0} \rightarrow \mathbb{C}$ hence defines a projective family of measures $\left(P_{J}^{\tilde{X}}\right)_{J \subseteq \mathbb{R}^{d}}$ finite;

(iv) There exists a canonical measure $P_{\text {can. }}^{\tilde{X}}$ on the space of functions $\omega: \mathbb{R}^{d} \rightarrow \mathbb{R}$ equipped with the sigma-algebra generated by pointwise evaluation $f \rightarrow f(x)$. The infinite volume interacting $C P N \tilde{X}(x)(\omega)=\omega(x)$ can be seen as the canonical process of $P_{\mathrm{can} .}^{\tilde{X}}$ in the above sense.

Proof. (i) This follows from Theorem 6.4 and Theorem 6.10,

(ii) One can use the representation through the dual correlation functional and one obtains the following uniform estimate

$$
\begin{aligned}
\left|\rho_{\Lambda}(\eta)-1\right| & =\left|\frac{1}{\Xi_{\Lambda}} \mathbb{E}_{P F}\left[e^{-\beta U_{\Lambda}\left(\eta+F_{\Lambda}\right)}-e^{-\beta U_{\Lambda}\left(F_{\Lambda}\right)}\right]\right| \\
& \leq \frac{1}{\Xi_{\Lambda}} \mathbb{E}_{P^{F}}\left[\left|e^{-\beta U_{\Lambda}\left(\eta+F_{\Lambda}\right)-\beta U_{\Lambda}\left(F_{\Lambda}\right)}-1\right| e^{-\beta U_{\Lambda}\left(F_{\Lambda}\right)}\right] \\
& \leq \sup _{\gamma \in \Gamma_{0}}\left|e^{-\beta U_{\Lambda}(\eta+\gamma)-\beta U_{\Lambda}(\gamma)}-1\right| \leq e^{\beta b\|G\|_{1} \sum_{l=1}^{n}\left|\alpha_{l}\right|}-1
\end{aligned}
$$

(iii) Thus, $\mathcal{C}_{\tilde{X}}$ defines a family of positive definite (as the limit of positive definite functions) and continuous functions that obviously generates a projective family of finite dimensional distributions $P_{J}^{\tilde{X}}$ of the random vectors $\left(X\left(x_{1}\right), \ldots, X\left(x_{n}\right)\right)$ for $J=\left\{x_{1}, \ldots, x_{n}\right\}$ (again, the projectivity property is evident for $\Lambda \subseteq \mathbb{R}^{d}$ 
compact and it survives the limit as the vectors converge in distribution by Lévy's theorem).

(iv) follows from (iii) and Kolmogorov's theorem on the existence of the inductive limit $P_{\text {can. }}^{\tilde{X}}$ of the family of finite dimensional distributions.

\section{The continuum limit}

In this section we discuss the continuum scaling limit of interacting particle systems with infra-red cut-off ${ }^{15}$. On the level of interacting CPNs, this scaling can be seen as a kind of implementation of the renormalization group.

\subsection{Scaling limits}

Here we first consider the situation of a gas of charged, noninteracting particles. The number of positiveandnegative charges is assumed to be equal in average, hence the gas macroscopically is neutral. If we let the number of particles per unit volume (the activity $z$ ) go to infinity $s$ scale charges with a factor $s \rightarrow s / \sqrt{z}$, we obtain the so-called continuum limit. See e.g. 23] for an overview over the scaling of particle systems.

Let $r$ be a probability measure on $[-c, c]$ s.t. $r\{0\}=0$. For $0<\lambda<\infty$ and $A \subseteq \mathbb{R}$ measurable, we define $r_{\lambda}(A)=r(\lambda A)$. Let $F$ be the Poisson noise determined by the Lévy measure $r$ and the activity $z=1$, cf. Eqs. (21) and (3). We then denote ${ }^{16}$ the Poisson noise determined by the Lévy measure $r_{1 / \sqrt{z}}$ and activity $z \geq 1$ by $F^{z}$. Throughout the section we assume $\int_{[-c, c]} s d r(s)=0$. We also set $\sigma^{2}=2 \int_{[-c, c]} s^{2} d r(s)$ and $\psi_{z}(t)=z \int_{[-c / \sqrt{z}, c / \sqrt{z}]}\left(e^{i s t}-1\right) d r_{1 / \sqrt{z}}(s)$. Finally, by $F_{g}^{\sigma}$ we denote the Gaussian noise with intensity $\sigma>0$ (cf. (2)-(3)) and we write $X^{z}=G * F^{z}, X_{g}^{\sigma}=G * F_{g}^{\sigma}$ for the associated convoluted Poisson and Gaussian noise, respectively. The basic facts on the continuum limit are given by the following proposition:

Proposition 7.1 With definitions as above we get

(i) $F^{z} \stackrel{\mathcal{L}}{\rightarrow} F_{g}^{\sigma}$ as $z \rightarrow \infty$;

(ii) $X^{z} \stackrel{\mathcal{L}}{\rightarrow} X_{g}^{\sigma}$ as $z \rightarrow \infty$.

Proof. We have (i) $\Leftrightarrow$ (ii) and it therefore suffices to prove the first statement. By Lévy's theorem convergence in law is equivalent with the convergence of characteristic functionals. It is thus sufficient to prove (cf. (22)-(3))

\footnotetext{
${ }^{15}$ Working with finite volume instead of an infra-red cut-off would lead to Gaussian tail fields outside this volume, which would lead to misleading "tail-effects" in the scaling.

${ }^{16}$ The superscript $z$ in this section is used in a different sense than in Section 6 , since there the charges remained unscaled.
} 
$\int_{\mathbb{R}^{d}} \psi_{z}(f) d x \rightarrow \int_{\mathbb{R}^{d}}\left(-\frac{\sigma^{2}}{2} f^{2}\right) d x$ as $z \rightarrow \infty \forall f \in \mathcal{S}$. Since $\psi_{z}(t) \rightarrow-\frac{\sigma^{2}}{2} t^{2}$ as $z \rightarrow \infty$ we have pointwise convergence and since $\left|\psi_{z}(f)\right| \leq \frac{\sigma^{2}}{2} f^{2} \forall z \geq 1$ the statement follows by dominated convergence.

We recall from Section 2.3 that Proposition 7.1 is of particular interest in the case $G=G_{\alpha, m_{0}}$, cf. Prop. 2.1. since then $X_{\alpha, m_{0}, g}^{\sigma}=G_{\alpha, m_{0}} * F_{g}^{\sigma}$ is a generalized free field for $0<\alpha<1 / 2$ and is Nelson's free field of mass $m_{0}>0$ for $\alpha=1 / 2$.

Next we investigate the effect of a length scale transformation $x \rightarrow \lambda x, x \in \mathbb{R}^{d}$, $0<\lambda<\infty$, on the Poisson noise $F$ and the $\operatorname{CPN} X_{\alpha, m_{0}}=G_{\alpha, m_{0}} * F$, respectively.

The basic observation is that increasing the activity can be performed by a scaling of the length $\left(z \sim \lambda^{d}\right)$, cf. Fig. 3.

Also one has to take into account that for a locally finite marked configuration we have a scaling dimension $\lambda^{-d}$, since $\delta(\lambda x)=\lambda^{-d} \delta(x)$. To obtain the same scaling as in Proposition 17.1, we thus have to define

$$
F_{\lambda}(x)=\lambda^{d / 2} F(\lambda x),
$$

$0<\lambda<\infty$, where this scaling relation has to be understood in the sense of distributions.

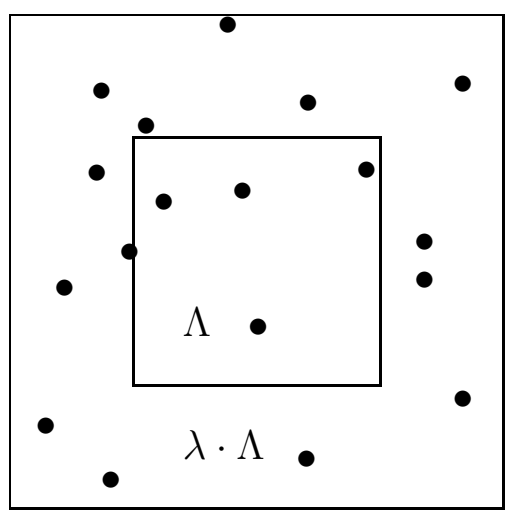

Figure 3: The average number of particles in a region $\Lambda$ is proportional to $|\Lambda|$. A scaling $\Lambda \rightarrow \lambda \Lambda$ thus scales the activity by a factor $\lambda^{d}$. Here $d=2, \lambda=2$.

Proposition 7.2 With definitions as above

(i) $F_{\lambda} \stackrel{\mathcal{L}}{=} F^{z}$ for $z=\lambda^{d}$;

(ii) For $X_{\alpha, m_{0}, \lambda}(x)=\lambda^{(d-4 \alpha) / 2} X_{\alpha, m_{0} / \lambda}(\lambda x)$ we get $X_{\alpha, m_{0}, \lambda} \stackrel{\mathcal{L}}{=} G_{\alpha, m_{0}} * F_{\lambda}$;

(iii) For $0<\alpha \leq 1 / 2, X_{\alpha, m_{0}, \lambda} \stackrel{\mathcal{L}}{\rightarrow} X_{\alpha, m_{0}, g}^{\sigma}$ as $\lambda \rightarrow \infty$ where the latter is a (generalized) free field.

Proof. (ii) follows from (i) and Proposition 2.1(v). (iii) follows from (i), (ii) and Proposition [7.1] To prove (i) let $f^{\lambda}(x)=f(x / \lambda), f \in \mathcal{S}, x \in \mathbb{R}^{d}, \lambda>0$. Then, by (171), $\left\langle F_{\lambda}, f\right\rangle=\lambda^{-d / 2}\left\langle F, f^{\lambda}\right\rangle$. Thus,

$$
\begin{aligned}
& \left.\mathcal{C}_{F_{\lambda}}(f)=e^{\int_{\mathbb{R}^{d}} \int_{[-c, c]}\left(e^{i s \lambda}-d / 2 f^{\lambda}(y)\right.}-1\right) d r(s) d y \\
& =e^{\lambda^{d} \int_{\mathbb{R}^{d}} \int_{\left[-\lambda-d / 2_{c, \lambda}-d / 2_{c]}\right.}\left(e^{i s f(y)}-1\right) d r_{\lambda-d / 2}(s) d y}, \quad f \in \mathcal{S},
\end{aligned}
$$

and the claim follows from Eqs. (22) and (3).

The scaling 7.2 (iii) is of the same form as for block-spin transformations implementing the renormalization group for lattice systems, [26]. In the general 
sense, that the renormalization group is a scaling limit adding more and more "microstructures" to a given region, we can say that the continuum limit for the models studied in this article is a suitable formulation of the renormalization group.

Remark 7.3 (i) It is an interesting fact that it is just property (ii) of Remark 2.2 which prevents us from taking a pointwise continuum limit: If we have $G \in$ $L^{2}\left(\mathbb{R}^{d}, d x\right)$, then the i.i.d. variables $Z_{j}(x)=S_{j} G\left(x-Y_{j}\right)$ with $S_{j}, Y_{j}$ distributed as $\left.r \otimes d x\right|_{\Lambda} /|\Lambda|$ for a finite volume $\Lambda \subseteq \mathbb{R}^{d}$ have finite variance $\frac{\sigma^{2}}{2} \int_{\Lambda}|G(x-y)|^{2} d y<$ $\infty$ and therefore fulfill the requirements of the central limit theorem. Under such conditions, the quantity $X_{\Lambda}^{z}(x)=\sum_{j=1}^{N_{\Lambda}^{z}} Z_{j}(x) / \sqrt{z}\left(N_{\Lambda}^{z}\right.$ being a Poisson random variable with intensity $z|\Lambda|$ ) converges in law to a Gaussian random variable, and one can thus expect a pointwise definition of the process $X_{\Lambda, g}^{\sigma}(x)$. If however $G \notin L^{2}\left(\mathbb{R}^{d}, d x\right)$, as it is the case in for the examples relevant for QFT, then the variance of $Z_{j}(x)$ is infinite. Heuristically speaking, $X_{\Lambda}^{z}(x)$ then converges to a "Gaussian random variable with infinite fluctuations" - thus there is no pointwise limit. Ultra-violet divergences and renormalization in these cases have to be taken into account. In the case $d=2, G=G_{1 / 2}$, the variance of $Z_{j}(x)$ only diverges logarithmically, which already gives a hint that ultra-violet divergences in this specific case ${ }^{17}$ will be rather mild.

(ii) From the above discussion it clear that the Gaussian (continuum) limit can also be taken in the canonical ensemble (CE) by replacing $N_{\Lambda}$ with it's expectation $|\Lambda|$. Interactions for the CE can be defined as in Section 4. It is however open, whether also the analytic continuation [3] can be performed in the CE. On the other hand, the $\mathrm{CE}$ is of advantage if one wants to work with potentials which might not be stable.

7.2 The continuum limit for trigonometric interactions with ultra-violet cut-off Here we study the continuum limit of CPNs with ultra-violet and infra-red regularized bounded interactions and we show convergence in law to the corresponding perturbed Gaussian models.

Let $G^{\epsilon}$ be a ultra-violet regularization of the kernel $G$ (cf. Section 5.3). $\Lambda \subseteq \mathbb{R}^{d}$ is assumed to be compact. Let $X_{\epsilon}^{z}=G^{\epsilon} * F^{z}$ and $X_{\epsilon}^{\sigma}=G^{\epsilon} * F^{\sigma}$. Here we dropped the superscripts $g$ for notational simplicity and we adopt the convention that a (convoluted) noise with superscript $\sigma$ is Gaussian. It is clear that $X_{\epsilon}^{\sigma}=\chi^{\epsilon} * X^{\sigma}$ has paths in the set $C^{\infty}\left(\mathbb{R}^{d}, \mathbb{R}\right)$. For $v: \mathbb{R} \rightarrow \mathbb{R}$ being measurable and bounded (by a constant $a>0$ ) we can thus define the potentials

$$
V_{\Lambda, \epsilon}^{z / \sigma}=\left\langle v\left(X_{\epsilon}^{z / \sigma}\right), 1_{\Lambda}\right\rangle .
$$

\footnotetext{
${ }^{17}$ This is the standard case considered usually in constructive QFT in two dimensions, see [1, 34, 55.
} 
and measures (for $\beta>0$, we also note that $\left|V_{\Lambda, \epsilon}^{z / \sigma}\right| \leq a|\Lambda|$ a.s.)

$$
P^{\bar{X}_{\epsilon, \Lambda}^{z / \sigma}}=\frac{e^{-\beta V_{\Lambda, \epsilon}^{z / \sigma}}}{\Xi_{\Lambda, \epsilon}^{z / \sigma}} P^{X_{\epsilon}^{z / \sigma}}, \quad \Xi_{\Lambda, \epsilon}^{z / \sigma}=\mathbb{E}_{P^{X_{\epsilon}^{z / \sigma}}}\left[e^{-\beta V_{\Lambda, \epsilon}^{z / \sigma}}\right]
$$

Let $\bar{X}_{\epsilon, \Lambda}^{z / \sigma}$ be the associated coordinate processes. We now obtain the same result as Proposition 7.1 for the perturbed models:

Theorem $7.4 \bar{X}_{\epsilon, \Lambda}^{z} \stackrel{\mathcal{L}}{\rightarrow} \bar{X}_{\epsilon, \Lambda}^{\sigma}$ as $z \rightarrow \infty$.

Proof. As convergence in law is equivalent with the convergence of characteristic functionals, we have to prove $\mathcal{C}_{\bar{X}_{\epsilon, \Lambda}^{z}}(f) \rightarrow \mathcal{C}_{\bar{X}_{\epsilon, \Lambda}^{\sigma}}(f) \forall f \in \mathcal{S}$. Since $V_{\Lambda, \epsilon}^{z}$ is an uniformly (in $z$ ) bounded random variable, we get that the expression

$$
\mathcal{C}_{\bar{X}_{\epsilon, \Lambda}^{z}}(f)=\frac{\sum_{n=0}^{\infty} \frac{(-\beta)^{n}}{n !} \mathbb{E}_{P^{X} \bar{\epsilon}}\left[e^{i X_{\epsilon}^{z}(f)}\left(V_{\Lambda, \epsilon}^{z}\right)^{n}\right]}{\sum_{n=0}^{\infty} \frac{(-\beta)^{n}}{n !} \mathbb{E}_{P^{X_{\epsilon}^{z}}}\left[\left(V_{\Lambda, \epsilon}^{z}\right)^{n}\right]}
$$

converges to the related expression with $z$ replaced with $\sigma$ if all terms in the numerator and denominator converge separately. Using Fubini's theorem we get for a term in the numerator

$$
\begin{aligned}
\mathbb{E}_{P^{X} X_{\epsilon}^{z}}\left[e^{i X_{\epsilon}^{z}(f)}\left(V_{\Lambda, \epsilon}^{z}\right)^{n}\right] & =\int_{\Lambda \times n} \mathbb{E}_{P^{X}}\left[e^{i X^{z}\left(\chi_{\epsilon} * f\right)}\right. \\
& \left.\times v\left(X^{z}\left(\chi_{\epsilon, y_{1}}\right)\right) \cdots v\left(X^{z}\left(\chi_{\epsilon, y_{n}}\right)\right)\right] d y_{1} \cdots d y_{n}
\end{aligned}
$$

and the corresponding term in the denominator is obtained setting $f=0$. Here $\chi_{\epsilon}$ is the ultra-violet cut-off function (cf. Section 5.3) and $\chi_{\epsilon, y}(x)=\chi_{\epsilon}(x-y)$. Since $\chi_{\epsilon, y} \in \mathcal{S}$ we now get the pointwise convergence of the integrand on the right hand side of (76) to the related integrand with $z$ replaced with $\sigma$ from the convergence in law of $X^{z}$, cf. Proposition[7.1(ii). Since the integrand is uniformly bounded by $(a|\Lambda|)^{n}$, convergence of the right hand side of (176) then follows from dominated convergence.

We want to modify this result in the following way: We replace the functions $v(t)$ in (173) with functions : $v(t):_{\epsilon}^{z / \sigma}=\int_{[-b, b]}: \cos (\alpha t):_{\epsilon}^{z / \sigma} d \nu(\alpha)$ where

$$
: \cos (\alpha t):_{\epsilon}^{z / \sigma}=\cos (\alpha t) / \mathbb{E}_{P^{X_{\epsilon}}} / \sigma\left[\cos \left(\alpha X_{\epsilon}^{z / \sigma}(x)\right)\right], \quad x \in \mathbb{R}^{d} .
$$

Here $\nu$ is a finite, complex measure on $[-b, b]$ s.t. $\nu(A)=\overline{\nu(-A)}$ for $A \subseteq$ $[-b, b]$ measurable. These energy densities define the (ultra-violet regularized) trigonometric interactions [9, 10]. It is not difficult to prove that under the given conditions : $v(t)::_{\epsilon}^{z}$ is uniformly bounded (in $z$ and $t$ ) and $: v(t):_{\epsilon}^{z} \rightarrow: v(t):_{\epsilon}^{\sigma}$ uniformly in $t$ as $z \rightarrow \infty$. Thus, the proof of Theorem 7.4 carries over to the modified interactions: 
Corollary 7.5 Let $\bar{X}_{\Lambda, \epsilon}^{z}$ be the ultra-violet regularized interacting $C P N$ with trigonometric interaction specified as above and let $\bar{X}_{\Lambda, \epsilon}^{\sigma}$ be the related perturbed Gaussian model. Then the statement of Theorem 7.4 still holds.

\subsection{Triviality for trigonometric potentials without renormalization}

We now want to consider the continuum limit without ultra-violet cut-off in the case of trigonometric potentials without renormalization ": : : ${ }_{0}^{\prime \prime}$, i.e. we set the denominator in (77) equal to one: Let $v(t)=\int_{\mathbb{R}} \cos (\alpha t) d \nu(\alpha)$ for some finite, complex measure $\nu$ on $\mathbb{R}$ such that $\nu\{0\}=0$ and $\nu(A)=\overline{\nu(-A)}$ for $A \subseteq \mathbb{R}$ measurable. Let furthermore $r$ be symmetric, $r(A)=r(-A), A \subseteq[-c, c]$ measurable. In this case $\psi_{z}$ is real and $\psi_{z}(t) \leq 0$. We chose $G \in L^{1}\left(\mathbb{R}^{d}, d x\right)$ such that $G \notin L^{2}\left(\mathbb{R}^{d}, d x\right)$, cf. Remarks 2.2 (ii) and 7.3 (i) for the motivation. Finally, we define $V_{\Lambda}^{z}$ as in Eq. (73) with $\epsilon=0$ and by Theorem 3.3 we get that this is well-defined (since $\nu$ is finite, $v$ is bounded). We get the following lemmma:

Lemma 7.6 $\left\|V_{\Lambda}^{z}\right\|_{L^{2}\left(\mathcal{S}^{\prime}, P^{X^{z}}\right)} \rightarrow 0$ as $z \rightarrow \infty$.

Proof. We get by Fubini's theorem for bounded functions

$$
\mathbb{E}_{P^{X^{z}}}\left[\left|V_{\Lambda}^{z}\right|^{2}\right]=\int_{\Lambda^{\times 2} \times \mathbb{R}^{2}} \mathbb{E}_{P^{X^{z}}}\left[e^{i\left(\alpha_{1} X^{z}\left(y_{1}\right)+\alpha_{2} X^{z}\left(y_{2}\right)\right)}\right] d y_{1} d y_{2} d \nu\left(\alpha_{1}\right) d \nu\left(\alpha_{2}\right)
$$

with (cf. Eq. (21) and Lemma 6.11)

$$
\mathbb{E}_{P^{X^{z}}}\left[e^{i\left(\alpha_{1} X^{z}\left(y_{1}\right)+\alpha_{2} X^{z}\left(y_{2}\right)\right)}\right]=e^{\int_{\mathbb{R}^{d}} \psi_{z}\left(\alpha_{1} G\left(x-y_{1}\right)+\alpha_{2} G\left(x-y_{2}\right)\right) d x}
$$

and the integral in the exponent on the right hand side exists for $0<z<\infty$, since $\left|\psi_{z}(t)\right| \leq c \sqrt{z}|t|$.

If we can show that the right hand side of (79) vanishes $d y_{1} d y_{2} d \nu\left(\alpha_{1}\right) d \nu\left(\alpha_{2}\right)$ a.e., we get the statement of the lemma by dominated convergence (since $\psi_{z} \leq 0$ ). Let $g(t)=\psi_{z}(\sqrt{z} t) / z t^{2}$. One easily verifies that $g$ is continuous and $g(0)=$ $-\sigma^{2} / 2$. By Fatou's lemma we get for $\alpha_{1}, \alpha_{2} \neq 0$ and $y_{1} \neq y_{2}$

$$
\begin{aligned}
& \limsup _{z \rightarrow \infty} \int_{\mathbb{R}^{d}} \psi_{z}\left(\alpha_{1} G\left(x-y_{1}\right)+\alpha_{2} G\left(x-y_{2}\right)\right) d x \\
&= \limsup _{z \rightarrow \infty} \int_{\mathbb{R}^{d}} g\left(\left[\alpha_{1} G\left(x-y_{1}\right)+\alpha_{2} G\left(x-y_{2}\right)\right] / \sqrt{z}\right) \\
& \times\left(\alpha_{1} G\left(x-y_{1}\right)+\alpha_{2} G\left(x-y_{2}\right)\right)^{2} d x \\
& \leq \int_{\mathbb{R}^{d}} \limsup _{z \rightarrow \infty} g\left(\left[\alpha_{1} G\left(x-y_{1}\right)+\alpha_{2} G\left(x-y_{2}\right)\right] / \sqrt{z}\right) \\
& \quad \times\left(\alpha_{1} G\left(x-y_{1}\right)+\alpha_{2} G\left(x-y_{2}\right)\right)^{2} d x \\
&=-\frac{\sigma^{2}}{2} \int_{\mathbb{R}^{d}}\left(\alpha_{1} G\left(x-y_{1}\right)+\alpha_{2} G\left(x-y_{2}\right)\right)^{2} d x=-\infty
\end{aligned}
$$


This concludes the proof.

Let $\bar{X}_{\Lambda}^{z}$ be the interacting CPN with infra-red cut-off $\Lambda$ associated to $V_{\Lambda}^{z}$. We then get:

Theorem $7.7 \bar{X}_{\Lambda}^{z} \stackrel{\mathcal{L}}{\rightarrow} X^{\sigma}$ as $z \rightarrow \infty$, i.e. the limit is trivial (Gaussian).

Proof. Again we have to show convergence of characteristic functionals. Let $f \in \mathcal{S}$, then

$$
\mathbb{E}_{P^{X^{z}}}\left[e^{i X^{z}(f)} e^{-\beta V_{\Lambda}^{z}}\right]=\mathcal{C}_{X^{z}}(f)-\mathbb{E}_{P^{X^{z}}}\left[e^{i X^{z}(f)}\left(1-e^{-\beta V_{\Lambda}^{z}}\right)\right]
$$

and from Lemma [7.6] we get

$$
\begin{aligned}
\left|\mathbb{E}_{P^{X^{z}}}\left[e^{i X^{z}(f)}\left(1-e^{-\beta V_{\Lambda}^{z}}\right)\right]\right| & \leq \mathbb{E}_{P^{X^{z}}}\left[\left|1-e^{-\beta V_{\Lambda}^{z}}\right|^{2}\right]^{1 / 2} \\
& \leq C \mathbb{E}_{P^{X^{z}}}\left[\left|V_{\Lambda}^{z}\right|^{2}\right]^{1 / 2} \rightarrow 0 \text { as } z \rightarrow \infty
\end{aligned}
$$

where $C=\beta|\Lambda||\nu|(\mathbb{R}) e^{\beta|\Lambda||\nu|(\mathbb{R})}$. Likewise one can show that $\Xi_{\Lambda}^{z} \rightarrow 1$ as $z \rightarrow \infty$. The statement now follows from Proposition 7.1 (ii).

Remark 7.8 (i) Clearly, Theorem 7.7 is what one would expect from the analysis of the sine-Gordon model [27, 28, 29]: The normal ordering : $\cos (\alpha t):{ }_{\epsilon}^{\sigma}$, cf. (77), in this case can be understood as a renormalization of the coupling constant, i.e. we chose the energy density $\cos (\alpha t)$ with coupling constant $\beta_{\epsilon}^{\sigma}=$ $\beta_{0} / \mathbb{E}_{P^{X_{\epsilon}^{\sigma}}}\left[\cos \left(\alpha X_{\epsilon}^{\sigma}(x)\right)\right]$ and one can easily check that $\beta_{\epsilon}^{\sigma} \uparrow \infty$ as $\epsilon \downarrow 0$. Since this coupling constant renormalization leads to a well-defined limit potential, it is natural to expect that without renormalization of $\beta$ the limit is trivial. This is the same statement as in Theorem [7.7. where we however use the continuum limit $z \rightarrow \infty$ without ultra-violet cut-off instead of the limit $\epsilon \downarrow 0$. We will continue this discussion in the following subsection.

(ii) Even though Theorem 7.7 does not come as a surprise, it's interpretation is of some interest: If $z \rightarrow \infty$ the spatial fluctuation of sample paths of $X^{z}$ increase rapidly, cf. Fig. 4. This leads to increasing oscillations of the function $\cos \left(\alpha X^{z}(x)\right)$ and thus $\int_{\Lambda} \cos \left(\alpha X^{z}(x)\right) d x$ integrates out to zero as $z \rightarrow \infty$.

(iii) For a different approach to the triviality of the sine-Gordon model without renormalization, based on random Colombeau distributions, see [8].

Another intresting approach to triviality in quantum field theory that is less motivated by trigonometric interactions than Remark 7.8 (ii) but probably works for all bounded non-renormalized interaction densities $v$ is to look at the "spatial" properties of the sample paths as depending on the strength of the ultra-violet singularity. Plots as in Fig. 3 at high scaling parameter are appropriate, cf. Fig. 


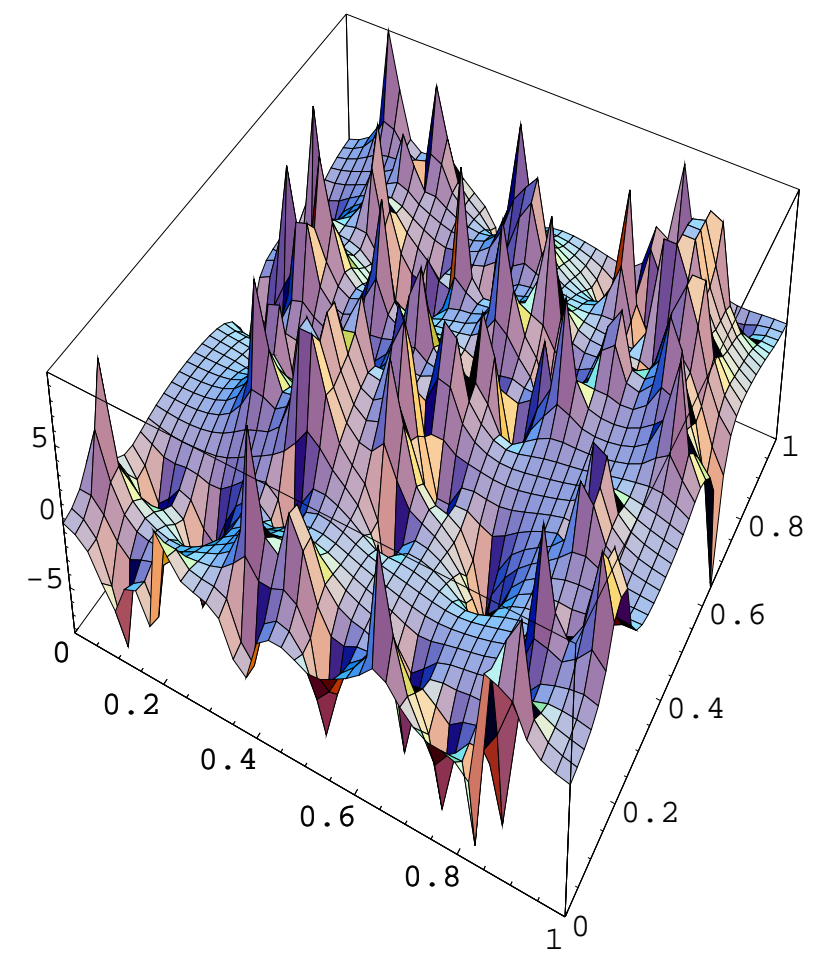

Figure 4: Sample paths of $X^{z}$ in the unit cube for $z=100$ (see also Fig. 1).

5. Already for $z=1000$ one can see that in the ultra-violet finite case (Fig. 5a) long range "Gaussian tails" dominate the sample path. Hence the fuctuations of the potential energy prevail in the scaling limit. In contrast to this, the ultraviolet divergent case exposes a strong "localization" of the path properties due to the "volume" of the singularities. Thus, each of the one hundred little squares with in average 10 particles is "approximately independent" from its neighbors and contributes an amount proportional to the covered volume $\sim 1 / 100$. One thus recognizes the regime of the law of large numers and the convergence of the potential to a constant (i.e. triviality of the interaction) is expected. Again, the uv-critical case (Fig. 5b) is just the uv-singularity strength of constructive quantum field theoryin $d=2$ dimensions.

\subsection{Some remarks on the continuum limit for the sine-Gordon model}

Here we give some remarks on the continuum limit for the sine-Gordon ( $\mathrm{sG}$ ) model in $d=2$ dimensions with coupling constant renormalization, namely we show that the Boltzmann weights of the dual particle system converge to those of the Yukawa gas, which is dual the sine-Gordon model, see e.g. [27]. We also comment on a simultanous expansion in the coupling constant $\beta$ and a rescaled activity $\zeta$ and we show that the continuum limit yields convergence in the 

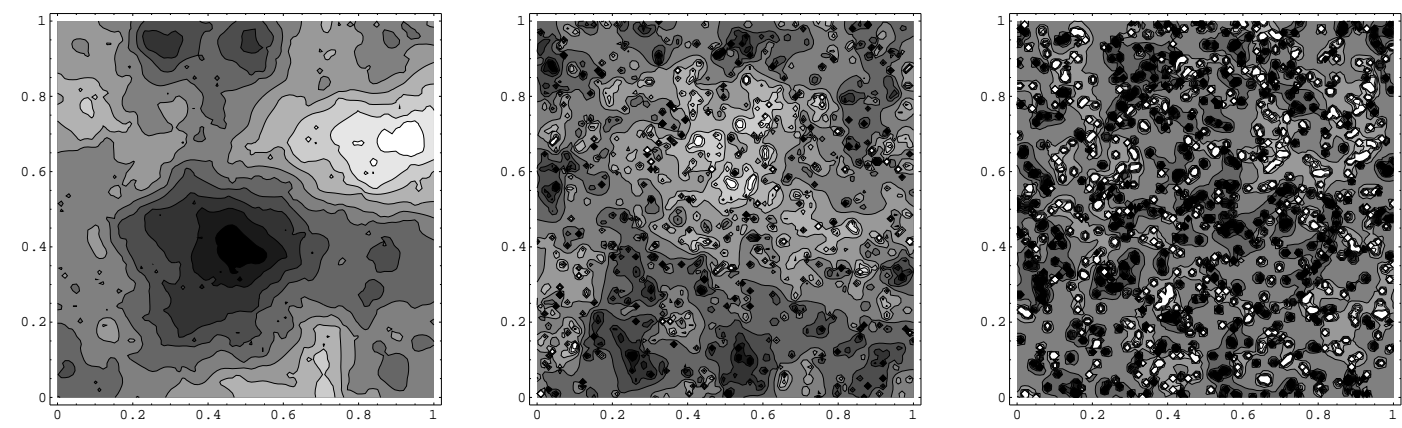

Figure 5: Density plot of the static field $\sim|x|^{-\alpha}$ of a two dimensional system of 1000 non-interacting charged particles a) for $\alpha=0.2$ (uv-finite scaling limit); b) $\alpha=1$ (uv-critical); c) $\alpha=2$ (uv-divergent).

expansion's coefficients. A treatment which goes beyond these very preliminary results and investigates convergence in law of the 'Poissonian' sine-Gordon models under the continuum limit would be desirable. But the technical details of such a treatment seem to be rather complicated, as it is case for the proof of the ultraviolet stability of the classical sine-Gordon model [24, 27, 28, 29]. It therefore goes beyond the scope of the present article.

We fix $G=G_{1 / 2, m_{0}}, G_{1}=G * G$, cf. Proposition 2.1. We consider the interacting CPN with energy density $: \cos (\alpha t):^{z}=: \cos (\alpha t):_{0}^{z}$, cf. (177). Using the language of particle systems, we define the potential for the dual particle system with external source $f$ as

$$
\begin{aligned}
U_{z}^{\mathrm{sG}}\left(f ; y_{1}, \ldots, y_{n} ; \alpha_{1}, \ldots, \alpha_{n}\right) & =\int_{\mathbb{R}^{d}}-\psi_{z}\left(G * f(x)+\sum_{l=1}^{n} \alpha_{l} G\left(x-y_{l}\right)\right) \\
& +\sum_{l=1}^{n} \psi_{z}\left(\alpha_{l} G\left(x-y_{l}\right)\right) d x
\end{aligned}
$$

where $f \in \mathcal{S}, y_{1}, \ldots, y_{n} \in \mathbb{R}^{d}, y_{j} \neq y_{l}, j \neq l$ and $\alpha_{1}, \ldots, \alpha_{n} \in \operatorname{supp} \nu \subseteq[-b, b]$. Here the integrals of the second term in (83) do not depend on the $y_{l}$ and these terms arise from the coupling constant renormalization (77). We also define

$$
\begin{aligned}
U_{\sigma}^{\mathrm{sG}}\left(f ; y_{1}, \ldots, y_{n} ; \alpha_{1}, \ldots, \alpha_{n}\right) & =\frac{\sigma^{2}}{2}\left[f * G_{1} * f(0)+2 \sum_{l=1}^{n} \alpha_{l} G_{1} * f\left(y_{l}\right)\right. \\
& \left.+\sum_{\substack{l, j=1 \\
j \neq l}}^{n} \alpha_{j} \alpha_{l} G_{1}\left(y_{j}-y_{l}\right)\right]
\end{aligned}
$$

which for $f=0$ gives the Yukawa potential for particles with charges $\alpha_{l}$. We consider the $f$-dependent Boltzmann weights $e^{-\zeta U_{z}^{\mathrm{sG}}\left(f ; y_{1}, \ldots, y_{n} ; \alpha_{1}, \ldots, \alpha_{n}\right)}$ for the dual 
particle system of the interacting CPN and $e^{-\zeta U_{\sigma}^{s \mathrm{G}}\left(f ; y_{1}, \ldots, y_{n} ; \alpha_{1}, \ldots, \alpha_{n}\right)}$ for the Yukawa gas. Here $\zeta>0$ is an inverse temperature for the dual particle systems and hence is a scaling factor for the activity (the intensity $\sigma$, respectively) for the quantum field systems, cf. Theorem 6.10. We get the following expansion in $\beta$ and $\zeta$ for the characteristic functional of $\bar{X}_{\Lambda}^{(z, \zeta)}$, defined as the interacting CPN with sG-interaction and Lévy-characteristic $\zeta \psi_{z}$ :

$$
\begin{aligned}
\mathcal{C}_{\bar{X}_{\Lambda}^{(z, \zeta)}}(f) & =\frac{1}{\Xi_{\Lambda}^{(z, \zeta)}} \sum_{l, n=0}^{\infty} \frac{(-\zeta)^{l}(-\beta)^{n}}{l ! n !} \int_{\left.\Lambda^{\times n} \times[-b, b]\right]^{\times n}} \\
& \times\left[U_{z}^{\mathrm{SG}}\left(f ; y_{1}, \ldots, y_{n} ; \alpha_{1}, \ldots, \alpha_{n}\right)\right]^{l} d y_{1} \cdots d y_{n} d \nu\left(\alpha_{1}\right) \cdots d \nu\left(\alpha_{n}\right) .
\end{aligned}
$$

The related expansion for the partition function is obtained from the expansion of the numerator by setting $f=0$. From the fact that $\mid U_{z}^{\mathrm{sG}}\left(f ; y_{1}, \ldots, y_{n} ; \alpha_{1}, \ldots\right.$, $\left.\alpha_{n}\right) \mid \leq C(n, z, c)$ where $C(n, z, c)$ is linearly bounded in $n$, we get that the expansion (85) converges absolutely for any fixed $z<\infty$, independently of the dimension $d$.

For $d=2$, the related expansion for the characteristic functional of the Gaussian sine-Gordon model exists term by term, which can be deduced from (84) and the fact that $G_{1}(x) \sim-\ln |x| / 2 \pi$ for $|x|$ small. It is known for the special case $\nu=\left(\delta_{b}+\delta_{-b}\right) / 2$ that if we sum up over $l$ under the integral, then the series converges absolutely for any $\beta$ provided $0<\zeta<2 / \sigma^{2} \sqrt{4 \pi b}$, cf. [27. From the analysis of that model it seems to us that after summing up $n$, at most asymptotic convergence in $l$ can be expected, since ultra-violet divergences for $\zeta<0$ are more severe than in the case $\zeta>0$. This can be explained from the fact that the Yukawa gas at negative temperatures becomes unstable. Here we ignore the question of convergence and consider (85) as a formal power series in $\beta$ and $\zeta$.

Proposition 7.9 With definitions as above

(i) The $f$-dependent Boltzmann weights (potentials $U_{z}^{\mathrm{sG}}$ ) of the dual particle system of the interacting CPN converge pointwisely to the $f$-dependent Boltzmann weights (potentials $U_{\sigma}^{\mathrm{sG}}$ ) of the Yukawa gas as $z \rightarrow \infty$.

(ii) For $d=2$ the expansion (85) converges to the related expansion of the classical ('Gaussian') sine-Gordon model, where $U_{z}^{\mathrm{sG}}$ is replaced by $U_{\sigma}^{\mathrm{sG}}$, in the sense of convergence of formal power series.

Proof. (i) Using $\psi_{z}(t) \rightarrow-\sigma^{2} t^{2} / 2$ as $z \rightarrow \infty$, it is elementary to show

$$
-\psi_{z}\left(\sum_{l=1}^{n} t_{l}+s\right)+\sum_{l=1}^{n} \psi_{z}\left(t_{l}\right) \rightarrow \frac{\sigma^{2}}{2}\left[\sum_{\substack{j, l=1 \\ l \neq j}} t_{l} t_{j}+2 s \sum_{l=1}^{n} t_{l}+s^{2}\right] \text { as } z \rightarrow \infty
$$


where $t_{1}, \ldots, t_{n}, s \in \mathbb{R}$. If we replace $t_{l}=\alpha_{l} G\left(x-y_{l}\right)$ and $s=G * f(x)$ we thus get the convergence of the left hand side of (86) to the right hand side whenever $x \neq y_{l}, l=1, \ldots, n$. We note that under this replacement, the right hand side of (86) integrated over $\mathbb{R}^{d}$ w.r.t. $d x$ is just the right hand side of (84). To prove the convergence of the right hand side of (83) to the right hand side of (84) for $y_{j} \neq y_{l}, l \neq j$, it is thus sufficient to show that the integrand in (83) has an uniform (in $z) L^{1}\left(\mathbb{R}^{d}, d x\right)$-bound.

We note that $\left|\psi_{z}(t)\right| \leq \sigma^{2} t^{2} / 2$ and $\left|\psi_{z}^{\prime}(t)\right| \leq \sigma^{2}|t|$ for all $z>0$. For $j=1, \ldots, n$ we thus get that the modulus of the left hand side of (86) can be estimated as follows:

$$
\begin{aligned}
\ldots & =\left|\int_{0}^{1} \psi^{\prime}\left(\left[\sum_{\substack{l=1 \\
l \neq j}}^{n} t_{l}+s\right] u+t_{j}\right)\left[\sum_{\substack{l=1 \\
l \neq j}}^{n} t_{l}+s\right]-\sum_{\substack{l=1 \\
l \neq j}} \psi_{z}\left(t_{j}\right) d u\right| \\
& \leq \sigma^{2} \sum_{\substack{l, p=1 \\
l, p \neq j}}^{n}\left|t_{l} t_{p}\right|+2 \sigma^{2} \sum_{\substack{l=1 \\
l \neq j}}^{n}\left|t_{l} s\right|+s^{2}+\frac{3 \sigma^{2}}{2} \sum_{\substack{l=1 \\
l \neq j}}^{n} t_{l}^{2}
\end{aligned}
$$

If one replaces on the right hand side $t_{l}$ with $\alpha_{l} G\left(x-y_{l}\right)$ and $s$ with $G * f(x)$ one apparently gets a function of fast decay which is locally integrable on $\mathbb{R}^{d} \backslash$ $\cup_{l=1, l \neq j}^{n} B_{R_{l}^{j}}\left(y_{l}\right)$ with $R_{l}^{j}=\left|y_{l}-y_{j}\right| / 2$ by our assumption $y_{j} \neq y_{l}, j \neq l$. A point $x \in \mathbb{R}^{d}$ is contained in such a set for $j$ s.t. the $\left|x-y_{j}\right|=\min \left\{\left|x-y_{l}\right|: l=1, \ldots, n\right\}$. Therefore, the union over $j=1, \ldots, n$ of all such sets gives $\mathbb{R}^{d}$ and there is a global $L^{1}\left(\mathbb{R}^{d}, d x\right)$-majorant.

(ii) To obtain the convergence in terms of formal power series in (85) it suffices to prove the convergence of each expansion coefficient in the numerator and in the denominator (i.e. in the expansion of $\Xi_{\Lambda}^{(z, \zeta)}$ ), since the coefficients of the expansion of the fraction can be calculated from those of the numerator and denominator via a finite combinatorial expression (note that the zero order coefficient of the partition function is one). Furthermore, the calculation for the partition function is a special case of the calculation for the numerator, namely $f=0$, we only have to consider the latter.

By (i) we have pointwise convergence of the integrands in (85). For $d=2, n$ and $l$ fixed, we can find a $L^{l}\left(\Lambda^{\times n} \times[-b, b]^{\times n}, d^{2 n} x \otimes r^{\otimes n}\right)$-majorant by integrating the majorant constructed in (i) over $\mathbb{R}^{2}$ w.r.t. $d x$. The fist term on the right hand side of (87) then gives rise to a term $\sum_{l, j=1, l \neq j}\left|\alpha_{l} \alpha_{j}\right| G_{1}\left(y_{l}-y_{j}\right)$ which is $L^{p}$-integrable in the variables $y_{1}, \ldots, y_{l}$ for any $p \geq 1$ since $G_{1}(x) \sim-\ln |x| / 2 \pi$ for small $x$. The terms involving $s$ and $s^{2}$ in (85) trivially have the same property, since under the replacements as above the integration over $d x$ can be estimated by $\sum_{l=1}^{n}\left|\alpha_{l}\right| G_{1} *|f|\left(y_{l}\right)$ and $|f| * G_{1} *|f|(0)$ which are manifestly bounded. Hence, the only really problematic term in (87) is the last one. 
This term, $\sum_{l=1, l \neq j}^{n} \alpha_{l}^{2} G\left(x-y_{l}\right)^{2}$, by the construction of the $d x$-majorant is integrated (in $x$ ) over $\mathbb{R}^{2} \backslash \cup_{l=1, l \neq j}^{n} B_{R_{l}^{j}}\left(y_{l}\right)$. By Proposition 2.1 (vi) applied to the case $d=2, \alpha=1 / 2$, one gets $|G(x)|<c_{1 / 2}(2) /|x|$. We can thus dominate this term by $-C_{1} \sum_{j, l=1 j \neq l}^{n} \ln \left(\left|y_{j}-y_{l}\right|\right) 1_{\left\{\left|y_{j}-y_{l}\right|<1\right\}}+n^{2} C_{2}$ for $C_{1}(\sigma, b), C_{2}(\sigma, b)>0$ sufficiently large. This establishes $L^{p}, p \geq 1$, integrability also for this last term and we can thus use the $d x$ integral of the majorant found in (i) as an $L^{l}$-majorant needed to prove dominated convergence in each term of (85).

Acknowledgments. Discussions with Klaus R. Mecke on Section 4.2 and Tobias Kuna on Section 6 were very helpful for the indicated parts of the article. We also thank Martin Grothaus, Armin Seyfried and Jiang-Lun Wu for interesting discussions and an anonymous referee for reading of the typoscript very carefully. Financial support for the second named author via DFG projects "Stochastic analysis and systems with infinitely many degrees of freedom" and "Stochastic methods in QFT" and for the third named author by the Grant-in-Aid Science Research No. 12640159 (Ministery of Education and Sciences, Japan) is gratefully acknowledged.

\section{References}

[1] S. Albeverio, J. E. Fenstad, R. Høegh-Krohn, T Lindstrøm: Nonstandard methods in stochastic analysis and mathematical Physics, Pure and Applied Math. 122, Academic Press, New York 1987.

[2] S. Albeverio, H. Gottschalk: Scattering theory for quantum fields with indefinite metric, Commun. Math. Phys. 216, p. 491 (2001).

[3] S. Albeverio, H. Gottschalk, J.-L. Wu, Convoluted generalized white noise, Schwinger functions and their continuation to Wightman functions, Rev. Math Phys., Vol 8, No. 6, p. 763, (1996).

[4] S. Albeverio, H. Gottschalk, J.-L. Wu, Models of local relativistic quantum fields with indefinite metric (in all dimensions), Commun. Math. Phys. 184, p. 509, (1997).

[5] S. Albeverio, H. Gottschalk, J.-L. Wu, Nontrivial scattering amplitudes for some local relativistic quantum field models with indefinite metric, Phys. Lett. B 405, p. 243 (1997).

[6] S. Albeverio, H. Gottschalk, J.-L. Wu, Scattering behavior of quantum vector fields obtained from Euclidean covariant SPDEs, Rep. on Math. Phys. 44 No. 1/2, p. 21 (1999).

[7] S. Albeverio, H. Gottschalk, M. W. Yoshida, Representing Euclidean Quantum fields as scaling limits of particle systems, Journ. Stat. Phys. 108, No. 1/2 631-639 (2002). 
[8] S. Albeverio, Z. Haba, F. Russo: A two-dimensional, semi-linear heat equation perturbed by white noise, Probab. Theory Relat. Fields 121, No.3, 319-366 (2001).

[9] S. Albeverio, R. Høegh-Krohn: Uniqueness of the physical vacuum and the Wightman functions in the infinite volume limit for some non-polynomial interactions. Commun. Math. Phys. 30, p. 171 (1973).

[10] S. Albeverio, R. Høegh-Krohn: The scattering matrix for some non-polynomial interactions I. Helv. Physica Acta 46, p. 504 (1973).

[11] S. Albeverio, R. Høegh-Krohn: Euclidean Markov fields and relativistic quantum fields from stochastic partial differential equations. Phys. Lett. B177, p. 175 (1986).

[12] S. Albeverio, R. Høegh-Krohn: Quaternionic non-Abelian relativistic quantum fields in four space-time dimensions. Phys. Lett. B189, p. 329 (1987).

[13] S. Albeverio, R. Høegh-Krohn: Construction of interacting local relativistic quantum fields in four space-time dimensions. Phys. Lett. B200, 108-114 (1988), with erratum in ibid. B202, p. 621 (1988).

[14] S. Albeverio, R. Høegh-Krohn, H. Holden, T. Kolsrud: Representation and construction of multiplicative noise. J. Funct. Anal. 87, p. 250 (1989).

[15] S. Albeverio, K. Iwata, T. Kolsrud: Random fields as solutions of the inhomogenous quarternionic Cauchy-Riemann equation. I. Invariance and analytic continuation , Commun. Math. Phys. 132 p. 550, (1990).

[16] S. Albeverio, J.-L. Wu: Euclidean random fields obtained by convolution from generalized white noise. J. Math. Phys. 36, 5217-5245 (1995)

[17] G. Battle, Wavelets and renormalization, World Scientific Sigapore/New Jersy/London/Hong Kong 1999

[18] H. Bauer: Mass- und Integrationstheorie, W. de Gruyter Berlin / New York 1990.

[19] C. Becker, R. Gielerak, P. Ługievicz: Covariant SPDEs and quantum field structures. J. Phys. A, p. 231 (1998).

[20] C. Berg, G. Forst: Potential Theory on Locally Compact Abelian Groups. Berlin/Heidelberg/New York: Springer-Verlag 1975.

[21] H.-J. Borchers, Algebraic aspects of quantum field theory, Int. Symp. math. Probl. theor. Phys., Kyoto 1975, Lect. Notes Phys. 39, 283-292 (1975).

[22] P. Colella, O. E. Lanford: Sample path behavior for the free Markov field. Lecture Notes Phys. 25 p. 44, Springer Berlin 1973.

[23] A. De Masi, E. Presutti: Mathematical methods for hydrodynamic limits. LNM 1501, Springer-Verlag Berlin / Heidelberg / New York 1991. 
[24] C. Deutsch, M. Lavaud: Equilibrium properties of a two-dimensional Coulomb gas. Phys. Rev. A 9, p. 2598 (1974).

[25] S.-H.Djah, H. Gottschalk, H. Ouerdiane, Feynman graph representation for the perturbation series for general functional measures, math-ph/0408031, to appear in Journ. Funct. Analysis.

[26] R. Fernàndez, J. Fröhlich, A. D. Sokal: Random Walks, Critical Phenomena and triviality in Quantum Field Theory, Berlin/Heidelberg/New York: Springer-Verlag 1992.

[27] J. Fröhlich: Classical and quantum statistical mechanics in one and two dimensions: Two-component Yukawa - and Coulomb systems. Commun. Math. Phys. 47, p. 233 (1976).

[28] J. Fröhlich, Y. M. Park: Remarks on exponential interactions and the quantum sine-Gordon equation in two space-time dimensions. Helv. Phys. Acta 50, p. 315 (1977).

[29] J. Fröhlich, E. Seiler: The massive Thirring-Schwinger model $(Q E D)_{2}$ : Convergence and perturbation structure. Helv. Phys. Acta 49, p. 889 (1976).

[30] I. M. Gelfand, N. Ya. Vilenkin: Generalized Functions, IV. Some Applications of Harmonic Analysis. New York/London: Academic Press 1964.

[31] H.-O. Georgii: Gibbs measures and phase transitions, W. de Gruyter, Berlin / New York 1988.

[32] R. Gielerak, P. Ługiewicz: From stochastic differential equation to quantum field theory, Rep. on Math. Phys. 44 No. 1/2, p. 101 (1999).

[33] R. Gielerak, P. Ługiewicz: 4D local quantum field theory models from covariant stochastic partial differential equations, Rev. Math. Phys. 13, No.3, 335-408 (2001).

[34] J. Glimm, A. Jaffe: Quantum Physics: A Functional Integral Point of View. 2nd ed. Berlin/Heidelberg/New York: Springer 1987.

[35] J. Glimm, A. Jaffe: Positivity of the $\phi_{3}^{4}$ Hamiltonian. Fortschr. Phys. 21, p. 327 (1973).

[36] O. W. Greenberg: Generalized Free Fields and Models of Local Field Theory. Annals of Physics 16 p. 158, (1969).

[37] H. Gottschalk, Particle systems with weakly attractive interaction, SFB 611 preprint Bonn 2002, math-ph/0409029.

[38] H. Gottschalk, Wick rotation for holomorphic random fields, in S. Albeverio, Z.-M. Ma, M. Rckner: "Recent developments in stochastic analysis and related topics" World Scientific, Singapore 2004, Proceedings of the First Sino-German Conference on Stochastic Analysis. 
[39] M. Grothaus, L. Streit: Construction of relativistic quantum fields in the framework of white noise analysis. J. Math. Phys. 40 No. 11, p. 5387 (1999).

[40] P. R. Halmos, Measure Theory, II.nd ed., Springer Verlag Berlin/Heidelberg 1976.

[41] G. E. Johnson: Interacting quantum fields. Rev. Math. Phys. 11 7, p. 881 (1999) with Erratum ibd. 12 p. 687 (2000).

[42] Yu. Kondratiev, T.Kuna, Correlation functionals for Gibbs Measures and Ruelle bounds, Methods Funct. Anal. Topol. 9, No.1, 9-58 (2003).

[43] A. Lenard: Correlation functions and the uniqueness of the state in classical statistical mechanics. Commun. Math. Phys. 30, p. 35 (1973).

[44] K. R. Mecke: Integral geometry in statistical physics. Int. Journ. Mod. Phys. 12 No. 9, p. 861 (1998).

[45] R. A. Minlos: Generalized random processes and their extension in measure. Translations in Mathematical Statistics and Probability , AMS Providence,3, p. 291 (1963).

[46] G. Morchio, F. Strocchi: Infrared singularities, vacuum structure and pure phases in local quantum field theory. Ann. Inst. H. Poincaré, 33, p. 251 (1980).

[47] E. Nelson: Construction of quantum fields from Markoff fields. J. Funct. Anal. 12, p. $97(1973)$

[48] E. Nelson: The free Markoff field. J. Funct. Anal. 12, p. 211 (1973)

[49] K. Osterwalder, R. Schrader: Axioms for Euclidean Green's functions I. Comm. Math. Phys. 31, p. 83 (1973)

[50] C. Preston: Random fields. LNM 534, Springer, Berlin / Heidelberg /New York, 1976.

[51] M. Reed, J. Rosen: Support properties of the free measure for the Boson field. Commun. Math. Phys. 36 p. 123 (1974).

[52] D. Ruelle: Statistical mechanics - rigorous results. Benjamin, London / Amsterdam / Don Mills (Ontario) / Sydney / Tokyo 1969.

[53] L. A. Santalò: Integral geometry and geometric probability. Addison-Wesley, Reanding (MA), 1976.

[54] B. Schroer: Infrateilchen in der Quantenfeldtheorie. Fortschr. Phys. 173 p. 1527 (1963).

[55] B. Simon: The $P(\phi)_{2}$ Euclidean (Quantum) Field Theory. Princeton University Press, Princeton, New Jersy, 1974. 
[56] D. Stoyan, W. S. Kendall, J. Mecke: Stochastic geometry and it's applications. Wiley \& Sons, 1987.

[57] F. Strocchi: Selected topics on the general properties of quantum field theory. Lecture Notes in Physics 51, World-Scientific Singapore / New York / London / Hong Kong, 1993.

[58] R. F. Streater, A. S. Wightman: PCT, spin, statistics and all that. New York: Benjamin 1964.

[59] K. Symanzik: Euclidean quantum field theory, in: Local Quantum Theory, Ed. R. Jost, Academic Press, New York 1969.

[60] H. Tamura: On the possibility of confinement caused by nonlinear electromagnetic interaction. J. Math. Phys. 32, p. 897 (1991)

[61] M. W. Yoshida: Non-linear continuous maps on abstract Wiener spaces defined on space of tempered distributions. Bulletin of the Univ. of Electro-Commun., 12 p. 101-117 (1999). 